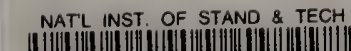

NBSIR 83-2788

\title{
Piece-Wise Analytic Evaluation of the Radiative Tail From Elastic and Inelastic Electron Scattering
}

U.S. DEPARTMENT OF COMMERCE

National Bureau of Standards

Center for Radiation Research

Washington, DC 20234

Nuclear Physics Laboratory

Department of Physics

University of Illinois at Urbana-Champaign

Urbana, lllinois 61801

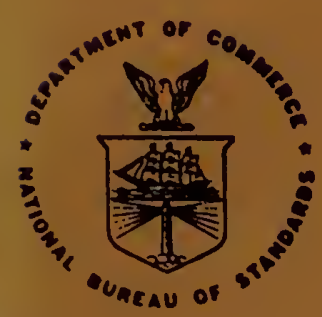

ac

U.S. DEPARTMENT OF COMMERCE

100

NATIONAL BUREAU OF STANDARDS

456

$33-2783$

1983

C. 2 

NBSIR $83-2788$

\section{PIECE-WISE ANALYTIC EVALUATION OF}

THE RADIATIVE TAIL FROM ELASTIC

AND INELASTIC ELECTRON SCATTERING

Leonard C. Maximon

U.S. DEPARTMENT OF COMMERCE

National Bureau of Standards

Center for Radiation Research

Washington, DC 20234

Steven E. Williamson

Nuclear Physics Laboratory

Department of Physics

University of Illionis at Urbana-Champaign

Urbana, Illinois 61801

December 1983

U.S. DEPARTMENT OF COMMERCE, Malcolm Baldrige, Secretary NATIONAL BUREAU OF STANDARDS. Ernest Ambler, Director 



\title{
PIECE-WISE ANALYTIC EVALUATION OF THE RADIATIVE TAIL \\ FROM ELASTIC AND INELASTIC ELECTRON SCATTERING
}

\author{
L. C. Maximon \\ National Bureau of Standards \\ Washington, D.C. 20234 \\ S. E. Williamson \\ Nuclear Physics Laboratory, Department of Physics \\ University of Illinois at Urbana-Champaign \\ Urbana, Illinois 61801
}

\section{ABSTRACT}

We consider here the calculation of the radiative tail from the elastic peak in medium and high energy electron scattering as well as from a discrete inelastic level of the recoiling nucleus. We examine the method generally used for this calculation, viz., a numerical integration of the differential cross section over the angles of the unobserved photon, and discuss the difficulties inherent in this numerical integration due to the sharp peaking of the integrand. We present an alternative method for calculating the radiative tail, in which the region of integration is divided into an arbitrary number of subintervals, the structure functions are fitted by cubic spline functions in each subinterval, and the integrations are then performed analytically in closed form. This method has the advantages of greatly increased accuracy and a reduction of the computation time by a factor which can vary between 10 and $10^{3}$, depending on the kinematics.

Key words: electron scattering cross sections; elastic electron scattering; elastic radiative tail; inelastic electron scattering; inelastic radiative tail; intermediate and high energy electron scattering. 


\section{ACKNOWLEDGEMENTS}

We wish to express our gratitude to Gloria Wiersma, to whom we are indebted for the skillful and expeditious typing of this report. We thank David K. Kahaner of the Scientific Computing Division at the National Bureau of Standards for suggesting the use of the Kronrod rules, embodied in the subroutines DQK61 and DIMACH. We also thank $\mathrm{Claude}$ Schuhl for the gracious hospitality accorded at the Centre d'Etudes Nucleaires de Saclay, where much of this work was done. This work was supported in part by the National Science Foundation under Grant NSF PHY 80-23603. 
CONTENTS

Abstract............................................

Acknowledgements......................................... i

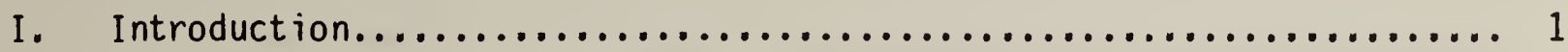

II. Preliminaries for the Piece-Wise Analytical Evaluation of the Radiative Tail....................................... 6

III. Details of the Piece-Wise Analytical Evaluation of the Radiative Tail 16

Appendix (Algebraic Details).

References.

Timing Table (Comparison of Piece-Wise Analytic Integration with Numerical

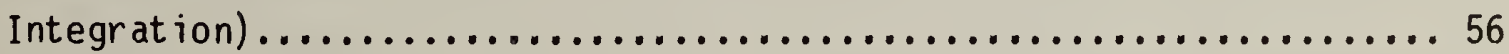

Computer Program Listing. 59 



\section{Introduction}

The analysis of medium and high energy electron scattering experiments involves, as is well known, the calculation of electromagnetic processes (e.g., radiative corrections, Landau straggling, radiative tails, etc.) which inevitably accompany and mask in some manner the electron-nucleus scattering of primary interest. The methods for evaluating the cross sections for these processes has been given in numerous articles and reviews familiar to experimenters, and are generally incorporated into the analys is of electron scattering experiments. We consider here the calculation of the radiative tail from the elastic peak, as weli as from a discrete inelastic level of the recoiling nucleus. 1 We examine the method which is generally used for this calculation, namely a numerical integration of the differential cross section over the angles of the unobserved photon, and discuss the difficulties inherent in this numerical integration. Finally, we present an alternative method for calculating the radiative tail having the advantage of greatly increased accuracy and a reduction of the computation time by a factor which can vary between 10 and $10^{3}$, depending on the kinematics (see Timing Table, p. 56).

The formalism used for the calculation of the radiative tail in almost all analyses of high-energy electron scattering experiments is that presented in a detailed series of papers by Tsai [1-3] ${ }^{2}$ and later refined by Miller [4]. In all of these papers the cross section for the scattering of an electron of initial momentum $\left|\underline{p} \underline{p}_{1}\right|$, final momentum $\left|\underline{p}_{2}\right|$ at an angle $\theta$, with emission of a photon, is given by an integral of the form

\footnotetext{
The assumption that the final state of the recoiling nucleus is a discrete level does not constitute a significant restriction in practice, since in the analysis a final continuum state is generally treated as a sum of many discrete levels.
}

${ }^{2}$ Figures in brackets indicate literature references at the end of this paper. 


$$
\frac{d^{2} \sigma}{d \Omega d\left|\underline{p}_{2}\right|}=\int\left\{W_{2} f_{2}+W_{1} f_{1}\right\} d \Omega_{k} .
$$

Here the functions $f_{2}$ and $f_{1}$ are known analytic functions of the initial and final electron momenta and scattering angle (which remain fixed) and the photon angles $\theta_{k}, \varphi_{k}$, over which one integrates. The structure functions $W_{2}$ and $W_{1}$ are, for elastic scattering, a function of the four-momentum transfer $q^{2}<0$. They are, in general, given as the result of a numerical calculation in which a charge distribution has been entered into a phase shift calculation. (That is, although a Born approximation formalism is used to derive the cross section given in (1), one uses, in the integrand in that expression, "experimental" structure functions, defined either by the experimental cross section divided by the Mott cross section or by a phase shift calculation.) It is expected, therefore, that the calculation of the radiative tail from (1) requires a numerical integration. However, the numerical integration of the expression appearing in the integrand in (1) is not completely trivial. Depending on the kinematics, the functions $f_{2}$ and $f_{1}$ can be very sharply peaked, - a reflection of the fact that the photons are emitted preferentially in the direction of either the initial or the final electron. In addition, since the functions $f_{2}$ and $f_{1}$ have an overall factor $\frac{1}{q^{4}}$, the integrand may rise sharply for $q^{2}$ near its minimum value, achieved (in the coordinate system defined following eqs (9)) when $\cos \theta_{k}=1$. These peaks are discussed in detail both in Maximon and Isabelle [5] and in Mo and Tsai [2]. In general, the height of the peaks is of order $\left(E / \mathrm{mc}^{2}\right)$ relative to the background and the width is of order $\left(\mathrm{mc}^{2} / \mathrm{E}\right)$ relative to the width of the region over which one integrates. The contribution from the peaks is thus of the same order of magnitude as that from the background, and for high energies 
great care must be taken to ensure that the numerical integration procedure has indeed converged and gives the desired accuracy. The result of the peaking is thus that the numerical integration may be quite lengthy, or of dubious accuracy, or both. Further, given the large number of intervals that must be chosen for the integration in the region of the peaks, the numerical evaluation of the structure functions $W_{2}$ and $W_{1}$ in each integration interval would be prohibitive in computation time, and rather useless since the functions $W_{2}$ and $W_{1}$ vary little over even the entire region of a peak. The procedure generally followed ${ }^{3}$ is therefore to divide the entire region of integration into a fixed number of subintervals $(1 \leqslant i \leqslant N$ ) (say with $N$ of the order of 100) and to fit $W_{2}$ and $W_{1}$ by spline functions in each of these subintervals, e.g., by a cubic function of $-q^{2}$ :

$$
\begin{aligned}
& w_{2}=a_{i}+b_{i}\left(-q^{2}\right)+c_{i}\left(-q^{2}\right)^{2}+d_{i}\left(-q^{2}\right)^{3} \\
& w_{1}=a_{i}^{\prime}+b_{i}^{\prime}\left(-q^{2}\right)+c_{i}^{\prime}\left(-q^{2}\right)^{2}+d_{i}^{\prime}\left(-q^{2}\right)^{3} .
\end{aligned}
$$

With the overall region of integration divided into these subintervals (some of which contain the peaks) and $W_{2}$ and $W_{1}$ represented by these simple analytic expressions in each subinterval, the numerical integration is then carried out as just discussed, the peak rendering the integration costly in time and questionable in accuracy.

${ }^{3}$ This is, essentially, the procedure followed by R. Altemus in the analys is of the data on ${ }^{56} \mathrm{Fe}$ taken at Bates (reported in Phys. Rev. Lett. 44, 965 (1980)), by M. Deady in the analys is of the data on ${ }^{40,48} \mathrm{Ca}$ taken at Bates (reported in Phys. Rev. C28, 631 (1983)), and by Z. Meziani in the analys is of the data on ${ }^{40,}{ }^{48} \mathrm{Ca}$ and ${ }^{56} \mathrm{Fe}$ taken at Saclay (to be published shortly). 
We note that the integration procedure just described consists in fact of two parts which are quite independent. In the first, each of the structure functions is fitted by a spline (or other useful function) in each of the $\mathrm{N}$ subintervals into which the integration region is subdivided for this purpose. (For example, for a point charge with spin zero we have $W_{2}=Z^{2}$ and $W_{1}=0$, so that we may choose $N=1$.) In the second part, the numerical integration of (1) is performed in each of the $N$ subintervals, with $W_{2}$ and $W_{1}$ now given by (2), using some suitable integration technique (e.g., Simpson's rule or Gaussian integration). In the event that a peak occurs in a given subinterval, then clearly this subinterval must be further subdivided, and the convergence of the integration procedure must be tested.

The essence of the present report is contained in the observation that once we have carried out the fitting of $W_{2}$ and $W_{1}$ by functions of the form given in (2) (the first part of the procedure just described), there is no longer any need to perform the integration numerically. With $W_{2}$ and $W_{1}$ given by finite polynomials in $q^{2}$ the functions $f_{2}$ and $f_{1}$ are sufficiently simple that the integration can be performed in each subinterval in closed form. (The resulting functions involve only algebraic functions and logarithms.) The integration over the entire region then consists merely of summing the contribution from each of the $N$ subintervals. The problems related to these peaks are thereby avoided completely. The presence of sharp peaks does not necessitate a further division of the subintervals. It is thus by eliminating the numerical integration, the second part of the procedure usually followed, that we reduce the time for the entire calculation by a factor which varies between 10 and $10^{3}$, depending on the specific kinematics. The accuracy of the entire calculation is also greatly increased, since it is now given by the precision of the computer rather than the accuracy of the particular numerical integration method. 
As a last general comment before entering into the details, we observe that in this note the method, which we call "piece-wise analytic" evaluation, is applied to the integration of the doubly differential cross section over the angles of the photon. However, it is equally applicable (with, of course, different resulting expressions) to the situation of interest for the analys is of experiments using photons as the electromagnetic probe. Then one integrates this same doubly differential cross section over the angles of the electron: The expressions which result in this latter case will be presented in a future report, in which we consider in particular the cross section for polarized photons (integrated over the angles of the final electron).

We next present the details of the calculation of the radiative tail given in this report. We divide this presentation into three parts: In the first we give preliminaries to the method presented here. Notation is defined, the differential cross section which forms the basis of our work is presented, and a few comments are made. In the second we give the details of the calculation as reflected directly in the computer program, a copy of which is included with this report. 4 Finally, in the Appendix we give details of the algebra involved in obtaining our final expression for the cross section, given by eq (38) and the subsidiary equations defining the quantities in it.

\footnotetext{
${ }^{4}$ The Fortran program is available from the authors. Requests should be accompanied by a magnetic tape and a specification of the desired format.
} 
II. Preliminaries for the Piece-Wise Analytical Evaluation of the Radiative Tail

The cross section under consideration here is the radiative tail to both elastic and inelastic scattering of electrons from a nucleus. In this report (as in the work of Tsai) we consider emission of photons only by the electron. Furthermore, the formalism used is that of the Born approximation. Thus in terms of diagrams we have
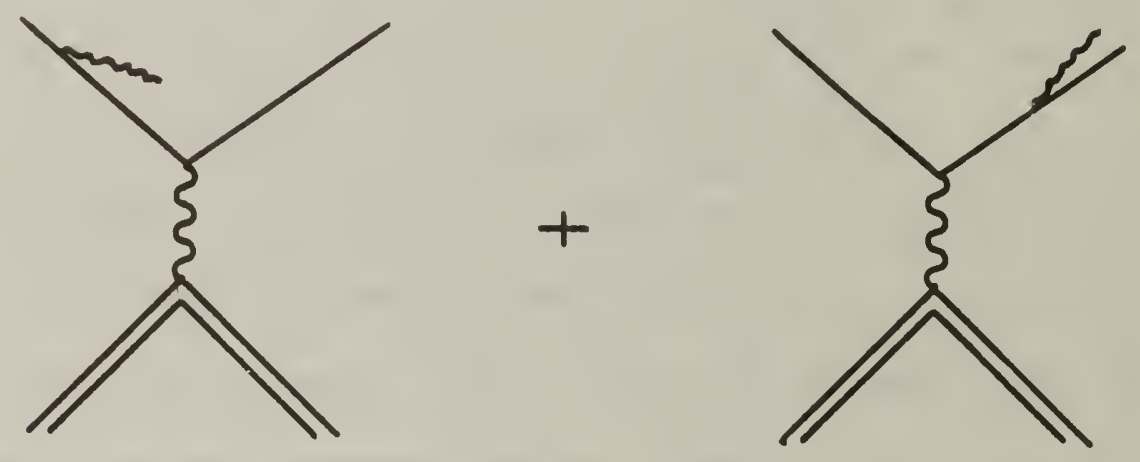

We do not consider here the contribution due to radiation by the recoiling nucleus. That has been treated in an approximate manner by Miller [4] and will be examined by us in a report to appear later.

The notation used here is as follows: Energies, momenta, and masses are all understood to be in energy units. When four-vectors are written, the metric is as in Bjorken and Drell [6], viz.,

$$
p_{1} \cdot p_{2}=\varepsilon_{1} \varepsilon_{2}-\underline{p}_{1} \cdot \underline{p}_{2}
$$




$$
\begin{aligned}
& \mathrm{p}_{1}=\left(\varepsilon_{1}, \underline{p}_{1}\right): \quad \text { four-momentum of the incident electron } \\
& \mathrm{p}_{2}=\left(\varepsilon_{2}, \underline{\mathrm{p}}_{2}\right): \quad \text { four-moment um of the scattered electron } \\
& k=(\omega, \underline{k}): \quad \text { four-momentum of the emitted (real) photon } \\
& q=\left(q_{0}, q\right) \text { : four-moment um of the virtual photon exchanged between } \\
& \text { the electron and the target particle } \\
& P_{1}=\left(E_{1}, \underline{P}_{1}\right): \quad \text { four-momentum of the target particle } \\
& =(M, 0) \quad \text { in the lab system } \\
& P_{2}=\left(E_{2}, P_{2}\right): \quad \text { four-momentum of the recoiling nucleus } \\
& =\left(M+q_{0}, \underline{q}\right) \quad \text { in the lab system } \\
& \mathrm{m}=\quad \text { rest mass of the electron } \\
& M=\quad \text { rest mass of the target particle. } \\
& M^{*}=\quad \text { rest mass of the final, recoiling nucleus } \\
& \mu \equiv \frac{M^{* 2}-M^{2}}{2 M} \quad\left(=\left(M^{*}-M\right)\left(1+\frac{M^{*}-M}{2 M}\right) \approx M^{*}-M \text { for } \frac{M^{*}-M}{2 M} \ll 1\right) \text {. (4) }
\end{aligned}
$$

$$
p_{1}+p_{1}=p_{2}+p_{2}+k \text {. }
$$

The momentum transferred to the nucleus is then

$$
q=p_{2}-p_{1}=p_{1}-p_{2}-k \text {. }
$$

Further, it is useful to define the four-vector $u$ by

$$
u=\left(u_{0}, \underline{u}\right) \equiv p_{1}+p_{1}-p_{2}=p_{2}+k
$$


Then in the lab system

$$
u_{0}=M+\varepsilon_{1}-\varepsilon_{2}
$$

and

$$
\underline{u}=\underline{p}_{1}-\underline{p}_{2}
$$

We note that $u_{0}$ and $\underline{u}$ are independent of both photon energy and angles.

$$
\begin{aligned}
& \theta=\text { scattering angle of the electron }\left(\theta=\Varangle\left(\underline{p}_{1}, \underline{p}_{2}\right)\right) \\
& \theta_{k}=\text { angle between } \underline{u} \text { and } \underline{k} \\
& \theta_{S}=\text { angle between } \underline{u} \text { and } \underline{p}_{1} \\
& \theta_{p}=\text { angle between } \underline{u} \text { and } \underline{p}_{2} .
\end{aligned}
$$

As in Mo and Tsai [2] the integration over photon angles is performed in a coordinate system such that the $z$ axis is in the direction of $\underline{u}=\underline{p}_{1}-\underline{p}_{2}$. As observed there, this coordinate system has the particular advantage that $q^{2}$ is then independent of the photon azimuthal angle $\varphi_{k}$. The structure functions $W_{2}\left(-q^{2}\right)$ and $W_{1}\left(-q^{2}\right)$ can then be taken outside of the integral over $\varphi_{k}$, which can then be performed analytically. We show this explicitely in the following paragraph.

From the notation just introduced, since the recoiling nucleus is assumed to be a free particle of mass $M^{\star}$, we have 


$$
\begin{aligned}
M^{\star 2}=P_{2}^{2} & =(u-k)^{2} \\
& =u^{2}-2 k \cdot u
\end{aligned}
$$

from which

$$
\omega=\frac{u^{2}-M^{\star 2}}{2\left(u_{0}-|\underline{u}| \cos \theta_{k}\right)} .
$$

Thus the photon energy, $\omega$, depends on the photon direction, but in the particular coordinate system we have chosen it is a function only of $\theta_{k}$, and not of $\varphi_{k}$. (Note, however, from the expression for $\omega$ given below, viz., $\omega=\varepsilon_{1}-\varepsilon_{2}-\mu+\frac{q^{2}}{2 M}$, that in the limit $\frac{q^{2}}{2 M} \rightarrow 0$, we have $\omega=\varepsilon_{1}-\varepsilon_{2}-\mu$; that is, in the absence of recoil the photon energy is independent of photon direction.)

Similarly, we can write

$$
\begin{aligned}
M^{\star 2}=P_{2}^{2} & =\left(P_{1}+q\right)^{2} \\
& =M^{2}+q^{2}+2 q \cdot P_{1} .
\end{aligned}
$$

Thus in the lab system

$$
q_{0}=-\frac{q^{2}}{2 M}+\mu
$$


On the other hand, substituting $q=p_{1}-p_{2}-k$ in $2 q \cdot p_{1}$ above gives

$$
2 p_{1} \cdot k=2 p_{1} \cdot\left(p_{1}-p_{2}\right)-M^{\star 2}+M^{2}+q^{2}
$$

so that in the lab system

$$
\omega=\varepsilon_{1}-\varepsilon_{2}-\mu+\frac{q^{2}}{2 M} .
$$

Thus in the coordinate system with z-axis in the direction of $\underline{u}$, both $w$ and $q^{2}$ are independent of $\varphi_{k}$. Only the products $p_{1} \cdot k$ and $p_{2} \cdot k$ depend on $\varphi_{k}$. Our starting point is then the doubly differential cross section for scattering of an electron with the emission of a photon, essentially as qiven by Tsai [1] and Mo and Tsai [2]. We make two minor changes in the expressions presented in these references, discussed immediately following the cross section written below.

$$
\left.\frac{d^{2} \sigma}{d \Omega d\left|\underline{p}_{2}\right|}=\frac{\alpha^{3}}{(2 \pi)^{2}} \frac{\left|\underline{p}_{2}\right|^{2}}{\varepsilon_{2}\left|\underline{p}_{1}\right|} \int_{-1}^{+1} \frac{2 M \omega d\left(\cos \theta_{k}\right)}{q^{4}\left(u_{0}-|\underline{u}| \cos \theta_{k}\right)} \int_{0}^{2 \pi} d \varphi_{k} \mid\right\}
$$

where 


$$
\begin{aligned}
& \{\}=W_{2}\left(-q^{2}\right)\left[-\frac{m^{2}}{\left(p_{1} \cdot k\right)^{2}}\left[2 \varepsilon_{2}\left(\varepsilon_{1}-w\right)+\frac{3}{2} q^{2}\right]-\frac{m^{2}}{\left(p_{2} \cdot k\right)^{2}}\left[2 \varepsilon_{1}\left(\varepsilon_{2}+w\right)+\frac{3}{2} q^{2}\right]\right. \\
& +\frac{2}{\left(p_{1} \cdot k\right)\left(p_{2} \cdot k\right)}\left(m^{2}\left(p_{1} \cdot p_{2}-\omega^{2}\right)+\left(p_{1} \cdot p_{2}\right)\left[2 \varepsilon_{1} \varepsilon_{2}-p_{1} \cdot p_{2}+\omega\left(\varepsilon_{1}-\varepsilon_{2}\right)\right]\right) \\
& -\frac{1}{\left(p_{1} \cdot k\right)}\left[2\left(\varepsilon_{1} \varepsilon_{2}-\varepsilon_{2} \omega+\varepsilon_{1}^{2}\right)+\frac{1}{2} q^{2}-p_{1} \cdot p_{2}-m^{2}\right] \\
& +\frac{1}{\left(p_{2} \cdot k\right)}\left[2\left(\varepsilon_{1} \varepsilon_{2}+\varepsilon_{1} \omega+\varepsilon_{2}{ }^{2}\right)+\frac{1}{2} q^{2}-p_{1} \cdot p_{2}-m^{2}\right] \\
& -2] \\
& +w_{1}\left(-q^{2}\right)\left[m^{2}\left(2 m^{2}+q^{2}\right)\left(\frac{1}{\left(p_{1} \cdot k\right)^{2}}+\frac{1}{\left(p_{2} \cdot k\right)^{2}}\right)\right. \\
& +\frac{4 p_{1} \cdot p_{2}\left(p_{1} \cdot p_{2}-2 m^{2}\right)}{\left(p_{1} \cdot k\right)\left(p_{2} \cdot k\right)} \\
& -\left(\frac{1}{\left(p_{1} \cdot k\right)}-\frac{1}{\left(p_{2} \cdot k\right)}\right)\left(2 p_{1} \cdot p_{2}+2 m^{2}-q^{2}\right) \\
& +4]
\end{aligned}
$$

The first modification we have made in the expression of Tsai concerns the structure functions. We use $W_{2}$ and $w_{1}$, defined by the elastic scattering cross section, which at high energies is given in terms of these functions by

$$
\frac{d \sigma_{e l}}{d \Omega}=\frac{\alpha^{2} \cos ^{2} \frac{1}{2} \theta\left[W_{2}+2 \tan ^{2} \frac{1}{2} \theta W_{1}\right]}{4 \varepsilon_{1}^{2} \sin ^{4} \frac{1}{2} \theta\left[1+\frac{2 \varepsilon_{1}}{M} \sin ^{2} \frac{1}{2} \theta\right]} \text {. }
$$


(Note that for a point charge with spin zero, $W_{2}(0)=z^{2}, W_{1}(0)=0$. ) This is the same as the change of notation introduced by Stein et al. [7] in Appendix $A$ of their paper. That appendix is a useful reference in that it is essentially a summary of Tsai's SLAC-PUR 848 [3]. The structure functions $F_{j}$ and $G_{j}$ used by Tsai are related to $W_{2}$ and $W_{1}$ by

$$
\begin{aligned}
& F_{j}=4 W_{2} \\
& G_{j}=4 M^{2} W_{1} .
\end{aligned}
$$

The second modification made in the expression of Tsai concerns the term $\frac{\left|\underline{p}_{2}\right|^{2}}{\varepsilon_{2}\left|\underline{p}_{1}\right|}$ which appears as a factor to the integral just written. In the articles of Tsai this factor has been replaced by its high energy limit, viz., $\frac{\varepsilon_{2}}{\varepsilon_{1}}$. While this replacement is clearly of little significance numerically for energies of experimental interest, we retain the factor $\frac{\left|\underline{p}_{2}\right|^{2}}{\varepsilon_{2}\left|\underline{p}_{1}\right|}$ out of a sense of consistency, since no high energy approximations are made anywhere else in the expression for the cross section. Our expression is then identical to that given by Nguyen-Ngoc and Perez-y-Jorba [8], apart from a trivial difference in the normalization of the structure functions: they use $F$ and $G$, which are related to our $W_{2}$ and $W_{1}$ by

$$
\begin{aligned}
& F=\frac{W_{2}}{M^{2}} \\
& G=-W_{1} .
\end{aligned}
$$


In any event, this term is closely related to the physics: Writing $\frac{\left|\underline{p}_{2}\right|^{2}}{\varepsilon_{2}\left|\underline{p}_{1}\right|}=\left(\frac{\left|\underline{p}_{2}\right|}{\varepsilon_{2}}\right) \frac{\left|\underline{p}_{2}\right|}{\left|\underline{p}_{1}\right|}$, we note that the factor $\frac{\left|\underline{p}_{2}\right|}{\varepsilon_{2}}$ comes from the fact that we have written the cross section as $\frac{d^{2} \sigma}{d \Omega d\left|\underline{p}_{2}\right|}$ rather than $\frac{d^{2} \sigma}{d \Omega d \varepsilon_{2}}$. The term $\left|\underline{p}_{1}\right|$ comes from the incident flux, and the final factor $\left|\underline{p}_{2}\right|$ comes from the density of final states for the scattered electron: (Note that in Stein et al. [7] they write $\frac{d^{2} \sigma}{d \Omega d \varepsilon_{2}}$, so that only $\frac{\left|\underline{p}_{2}\right|}{\left|\underline{p}_{1}\right|}$ should appear, but they replace this with $\frac{\varepsilon_{2}}{\varepsilon_{1}}$.) This factor without high energy approximation has also been retained to facilitate comparison with the Bethe-Heitler cross section in the limit $M+\infty$. Finally, we note that in Stein et al. [7] the structure functions $W_{2}$ and $W_{1}$ have been replaced by $\widetilde{W}_{2}$ and $\widetilde{W}_{1}$, defined by (A42) and (A43) of Stein et al.:

$$
\begin{aligned}
& \tilde{W}_{2}\left(q^{2}\right)=\tilde{F}\left(q^{2}\right) W_{2}\left(q^{2}\right) \\
& \tilde{W}_{1}\left(q^{2}\right)=\tilde{F}\left(q^{2}\right) W_{1}\left(q^{2}\right)
\end{aligned}
$$

to account for both thick target bremsstrahlung (equivalent radiator) and that part of the radiative correction which does not depend on the resolution $\Delta E$. In addition, in the work of Miller [4] these factors are further modified to account (in an approximate manner) for the multiple emission of soft photons from both the electron and the target particle. The important point to retain here is that all these factors depend on the photon direction only through the four-momentum transfer $q^{2}$ (or via the photon energy, which is again a linear function of $q^{2}$, as we have just seen). All these effects can therefore be accounted for simply by an appropriate modification of the structure functions which leaves them independent of $\varphi_{k}$. 
We next perform the integration over $\varphi_{k}$, as in Mo and Tsai [2], Tsai [3], and Stein et al. [7], and obtain

$$
\frac{d^{2} \sigma}{d \Omega d\left|\underline{p}_{2}\right|}=\frac{\alpha^{3}}{2 \pi} \frac{\left|\underline{p}_{2}\right|^{2}}{\varepsilon_{2}\left|\underline{p}_{1}\right|} \int_{-1}^{+1} \frac{2 M \omega d\left(\cos \theta_{k}\right)}{q^{4}\left(u_{0}-|\underline{u}| \cos \theta_{k}\right)}\{\}
$$

where

$$
\begin{aligned}
& \{\}=W_{2}\left(-q^{2}\right)\left[-\frac{a m^{2}}{x^{3}}\left[2 \varepsilon_{1}\left(\varepsilon_{2}+\omega\right)+\frac{1}{2} q^{2}\right]-\frac{a^{\prime} m^{2}}{y^{3}}\left[2 \varepsilon_{2}\left(\varepsilon_{1}-\omega\right)+\frac{1}{2} q^{2}\right]\right. \\
& +2 v\left(\frac{1}{x}-\frac{1}{y}\right)\left\{m^{2}\left(p_{1} \cdot p_{2}-w^{2}\right)+\left(p_{1} \cdot p_{2}\right)\left[2 \varepsilon_{1} \varepsilon_{2}-p_{1} \cdot p_{2}+w\left(\varepsilon_{1}-\varepsilon_{2}\right)\right]\right\} \\
& +\frac{1}{x}\left[2\left(\varepsilon_{1} \varepsilon_{2}+\varepsilon_{1} \omega+\varepsilon_{2}^{2}\right)+\frac{1}{2} q^{2}-p_{1} \cdot p_{2}-m^{2}\right] \\
& -\frac{1}{y}\left[2\left(\varepsilon_{1} \varepsilon_{2}-\varepsilon_{2} \omega+\varepsilon_{1}{ }^{2}\right)+\frac{1}{2} q^{2}-p_{1} \cdot p_{2}-m^{2}\right] \\
& -2] \\
& +W_{1}\left(-q^{2}\right)\left[\left(\frac{a}{x^{3}}+\frac{a^{\prime}}{y^{3}}\right) m^{2}\left(2 m^{2}+q^{2}\right)+4 v\left(\frac{1}{x}-\frac{1}{y}\right)\left(p_{1} \cdot p_{2}\right)\left(p_{1} \cdot p_{2}-2 m^{2}\right)\right. \\
& \left.+\left(\frac{1}{x}-\frac{1}{y}\right)\left(2 p_{1} \cdot p_{2}+2 m^{2}-q^{2}\right)+4\right]
\end{aligned}
$$


in which 5,6

$$
\begin{aligned}
a & =\omega\left(\varepsilon_{2}-\left|\underline{p}_{2}\right| \cos \theta_{p} \cos \theta_{k}\right) \\
a^{\prime} & =\omega\left(\varepsilon_{1}-\left|\underline{p}_{1}\right| \cos \theta_{s} \cos \theta_{k}\right) \\
b & =-\underline{w}_{2} \mid \sin \theta_{p} \sin \theta_{k} \\
\nu & =\left(a^{\prime}-a\right)^{-1} \\
x & =\left(a^{2}-b^{2}\right)^{1 / 2} \\
y & =\left(a^{\prime 2}-b^{2}\right)^{1 / 2} \\
\cos \theta_{s} & =\frac{\left|\underline{p}{ }_{1}\right|-\left|\underline{p}{ }_{2}\right| \cos \theta}{|\underline{u}|} \\
\cos \theta_{p} & =\frac{\left|\underline{p} \underline{1}_{1}\right| \cos \theta-\left|\underline{p} \underline{p}_{2}\right|}{|\underline{u}|} \\
\underline{p}^{\prime} &
\end{aligned}
$$

${ }^{5}$ The symbol $x$ which is defined in (24) and used in (23) is employed at this point out of consistency with the notation of Tsai [3] and Stein et al. [7]. It is used nowhere else in this report as defined in (24). Elsewhere throughout this report we define $x=-q^{2}$.

${ }^{6}$ The expressions given here in (24) are those given in Tsai's SLAC-PUB 848 [3] and Stein et al. [7]. They represent a considerable simplification of the expressions given originally in Mo and Tsai [2]. 
III. Details of the Piece-Wise Analytical Evaluation of the Radiative Tail

At this point we depart from the procedure followed in these references. Since we perform the remaining integration analytically, the most appropriate variable of integration is $q^{2}$ rather than $\cos \theta_{k}$, in view of the functions $W_{2}\left(-q^{2}\right)$ and $W_{1}\left(-q^{2}\right)$. Indeed, the form of the integrand itself is then somewhat simpler. To give just one example, referring to the overall factor in the integrand in above expressions for the radiative tail, we now have, with $q^{2}$ as integration variable,

$$
\frac{2 M \omega d\left(\cos \theta_{k}\right)}{q^{4}\left(u_{0}-|\underline{u}| \cos \theta_{k}\right)}=\frac{1}{|\underline{u}|} \frac{d\left(q^{2}\right)}{q^{4}} \text {. }
$$

We will not, however, encumber this section of the report with these algebraic manipulations. They are presented at the end in the Appendix. Here we give only the final form of our expression for the radiative tail, which lends itself directly to the computer implementation of the calculation. As variable of integration we choose finally a positive quantity, viz.,

$$
x=-q^{2}>0
$$

The cross section for the radiative tail is then given by

$$
\frac{d^{2} \sigma}{d \Omega d\left|\underline{p}_{2}\right|}=\frac{\alpha^{3}}{\pi} \frac{\left|\underline{p}_{2}\right|^{2}}{\varepsilon_{2}\left|\underline{p}_{1}\right|} \int_{x_{m}}^{x_{M}} d x\left[W_{2}(x)\{\}+W_{1}(x)\{\}^{\prime}\right]
$$


where

$$
\begin{aligned}
\mid f= & -\frac{1}{x^{2} D_{1}^{3 / 2}(x)}\left[\beta_{0}+\beta_{1} x+\beta_{2} x^{2}\right] \\
& -\frac{1}{x^{2} D_{2}^{3 / 2}(x)}\left[\delta_{0}+\delta_{1} x+\delta_{2} x^{2}\right] \\
& -\frac{\eta u^{2} x^{2}}{(2 \lambda-x)}\left(\frac{1}{D_{1}{ }^{1 / 2}(x)}-\frac{1}{D_{2}{ }^{1 / 2}(x)}\right) \\
& +\frac{\tau_{1}}{x^{2} D_{1}^{1 / 2}(x)}-\frac{\tau_{2}}{x^{2} D_{2}^{1 / 2}(x)} \\
& -\frac{\rho_{1}}{x D_{1}^{1 / 2}(x)}+\frac{\rho_{2}}{x D_{2}^{1 / 2}(x)}
\end{aligned}
$$

and where \{\}$^{\prime}$ is obtained from \{\} merely by replacing the parameters $\beta_{0}, \beta_{1}, \ldots$ by the corresponding parameters with primes:

$$
\begin{gathered}
\left\{\beta_{0}, \beta_{1}, \beta_{2}, \delta_{0}, \delta_{1}, \delta_{2}, \eta, \gamma, \tau_{1}, \tau_{2}, \rho_{1}, \rho_{2}\right\} \\
+\left\{\beta_{0}^{\prime}, \beta_{1}^{\prime}, \beta_{2}^{\prime}, \delta_{0}^{\prime}, \delta_{1}^{\prime}, \delta_{2}^{\prime}, \eta^{\prime}, \gamma^{\prime}, \tau_{1}^{\prime}, \tau_{2}^{\prime}, \rho_{1}^{\prime}, \rho_{2}^{\prime}\right\}
\end{gathered}
$$

In (28) the quadratic functions $D_{1}(x)$ and $D_{2}(x)$ are related to the quantities $a$, $a^{\prime}$, and $b$ of $(24)$ by eqs $(A 19)$ and $(A 25)$. Referring to the terms in the integrand with factor $\frac{1}{(2 \lambda-x)}$, we note in the appendix that the point $x=2 \lambda$ indeed lies in the range of integration:

$$
x_{m}<2 \lambda<x_{M} \text {, }
$$


but that $D_{1}(2 \lambda)=D_{2}(2 \lambda)$, so that there is in fact no singularity in the integrand at the point $x=2 \lambda$. (This is shown explicitely later in this report, in the Appendix.)

The parameters appearing in the integral in (27) and (28) are defined shortly below. However, we first outline our piece-wise analytic evaluation of the cross section. We divide the range of integration $\left(x_{m}, x_{M}\right)$ into $N$ subintervals (which can be of different and arbitrary length) writing

$$
\int_{x_{m}}^{x_{M}} d x \ldots=\sum_{i=1}^{N} \int_{x_{i-1}}^{x_{i}} d x \ldots
$$

with

$$
x_{0}=x_{m} \quad, \quad x_{N}=x_{M} .
$$

In each subinterval $\left(x_{i-1}, x_{j}\right)$ we represent $W_{2}(x)$ and $W_{1}(x)$ by a third order polynomial in $x$ : The coefficients of the polynomial will, in general, be different in each subinterval.

In $\left(x_{i-1}, x_{i}\right)$

$$
\begin{aligned}
& w_{2}(x)=a_{i}+b_{i} x+c_{i} x^{2}+d_{i} x^{3} \\
& w_{1}(x)=a_{i}^{\prime}+b_{i}^{\prime} x+c_{j}^{\prime} x^{2}+d_{i}^{\prime} x^{3}
\end{aligned}
$$

We may then write 


$$
\begin{aligned}
\int_{x_{m}}^{x_{M}} d x\left[w_{2}(x)\{\}+w_{1}(x)\{\}^{\prime}\right] & =\sum_{i=1}^{N} \int_{x_{i-1}}^{x_{i}} d x\left[w_{2}(x)\{\}+w_{1}(x)\{\}^{\prime}\right] \\
& =\sum_{i=1}^{N} \int_{x_{i-1}}^{x_{i}} d x\left[\left(a_{i}+b_{i} x+c_{i} x^{2}+d_{j} x^{3}\right)\{\}\right. \\
& +\left(a_{i}^{\prime}+b_{i}^{\prime} x+c_{i}^{\prime} x^{2}+d_{i}^{\prime} x^{3}\right)\{\}
\end{aligned}
$$

Considering the expressions just given for \{\} and \{\}$^{\prime}$ (the functions $D_{1}(x)$ and $D_{2}(x)$ are quadratic functions of $x$ ), the integrals can now all be performed analytically. The integrations are all straightforward - none result in functions any more complicated than the logarithm. However, care must be taken in arranging the terms, and in particular the arguments of the logarithms, so that at high energies there are no cancellations between almost equal terms, leaving a remainder of relative order $\left(m / \varepsilon_{1}\right)^{2}$, with a consequent loss in precision.

Referring to the integrand, the integrals to be performed analytically are thus of the the form

$$
\begin{aligned}
& I_{1, i}^{(1)}=\int_{x_{i-1}}^{x_{i}} \frac{d x}{x^{2} D_{1}^{3 / 2}(x)}\left[a_{i}+b_{i} x+c_{i} x^{2}+d_{i} x^{3}\right]\left[\beta_{0}+\beta_{1} x+\beta_{2} x^{2}\right] \\
& I_{i}^{(2)}=\int_{x_{i-1}}^{x_{i}} \frac{d x}{x^{2}}\left[a_{i}+b_{i} x+c_{i} x^{2}+d_{i} x^{3}\right] \\
& I_{1, i}^{(3)}=\int_{x_{i-1}}^{x_{i}} \frac{d x}{x^{2} D_{1}^{1 / 2}(x)}\left[a_{i}+b_{i} x+c_{i} x^{2}+d_{i} x^{3}\right]
\end{aligned}
$$




$$
I_{1, i}^{(4)}=\int_{x_{i-1}}^{x_{i}} \frac{d x}{x D_{1}^{1 / 2}(x)}\left[a_{i}+b_{i} x+c_{i} x^{2}+d_{i} x^{3}\right]
$$

and

$$
I_{i}^{(5)}=\int_{x_{i-1}}^{x_{i}} \frac{d x}{(2 \lambda-x)}\left(\frac{1}{D_{1}^{1 / 2}(x)}-\frac{1}{D_{2}^{1 / 2}(x)}\right)\left[a_{i}+b_{i} x+c_{i} x^{2}+d_{i} x^{3}\right] \cdot \text { (34e) }
$$

The integrals $I_{2, i}^{(1)}, I_{2, i}^{(3)}$, and $I_{2, i}^{(4)}$ follow from $I_{1, i}^{(1)}, I_{1, i}^{(3)}$, and $I_{1, i}^{(4)}$ merely by replacing $D_{1}(x)$ by $D_{2}(x)$ in the integrands (and replacing $\beta_{0}, \beta_{1}, \beta_{2}$ in $I_{1, i}^{(1)}$ by $\left.\delta_{0}, \delta_{1}, \delta_{2}\right)$. We then write

$$
\begin{aligned}
I_{i}= & -I_{1, i}^{(1)}-I_{2, i}^{(1)}-\frac{\eta}{|\underline{u}|} I_{i}^{(2)}+\tau_{1} I_{1, i}^{(3)}-\tau_{2} I_{2, i}^{(3)} \\
& -\rho_{1} I_{1, i}^{(4)}+\rho_{2} I_{2, i}^{(4)}-\gamma I_{i}^{(5)}
\end{aligned}
$$

and define $I_{j}^{\prime}$ by substituting throughout the corresponding parameters with primes:

$$
\left\{\beta_{0}, \beta_{1}, \ldots\right\}+\left\{\beta_{0}^{\prime}, \beta_{1}^{\prime}, \ldots\right\}
$$

and

$$
\left\{a_{i}, b_{i}, c_{i}, a_{j}\right\}+\left\{a_{j}^{\prime}, b_{i}^{\prime}, c_{i}^{\prime}, d_{j}^{\prime}\right\}
$$

The integral given for the cross section is then

$$
\int_{x_{m}}^{x_{M}} d x \ldots=\sum_{i=1}^{N}\left[I_{i}+I_{j}^{\prime}\right]
$$


and our piece-wise analytic expression for the cross section is

$$
\frac{d^{2} \sigma}{d \Omega d\left|\underline{p}_{2}\right|}=\frac{\alpha^{3}}{\pi} \frac{\left|\underline{p}_{2}\right|^{2}}{\varepsilon_{2}\left|\underline{p}_{1}\right|} \sum_{i=1}^{N}\left[I_{i}+I_{j}^{\prime}\right] .
$$

Again we stress that the integrations are all performed analytically. The sum above reflects only the subdivision such that $W_{2}(x)$ and $W_{1}(x)$ can be approximated with sufficient accuracy by third order polynomials in each subinterval. $I_{i}^{(5)}$, we see that the important integrals to be evaluated are

$$
\text { Referring to the integrals } I_{1, i}^{(1)}, I_{2, i}^{(1)}, I_{i}^{(2)}, I_{1, i}^{(3)}, I_{2, i}^{(3)}, I_{1, i}^{(4)}, I_{2, i}^{(4)} \text {, and }
$$

$$
\begin{aligned}
& J_{1, m}=\int_{x_{i-1}}^{x_{i}} \frac{x^{m}}{D_{1}^{1 / 2}(x)} d x \\
& J_{2, m}=\int_{x_{i-1}}^{x_{i}} \frac{x^{m}}{D_{2}^{1 / 2}(x)} d x \\
& k_{1, m}=\int_{x_{i-1}}^{x_{i}} \frac{x^{m}}{D_{1}^{3 / 2}(x)} d x \\
& k_{2, m}=\int_{x_{i-1}}^{x_{i}} \frac{x^{m}}{D_{2}^{3 / 2}(x)} d x
\end{aligned}
$$


and

$$
L=\int_{x_{i-1}}^{x_{i}} \frac{1}{(2 \lambda-x)}\left(\frac{1}{D_{1}^{1 / 2}(x)}-\frac{1}{D_{2}^{1 / 2}(x)}\right) d x
$$

In terms of these integrals we have

$$
\begin{aligned}
& I_{1, i}^{(1)}=a_{i} \beta_{0} K_{1,-2}+\left(b_{i} \beta_{0}+a_{i} \beta_{1}\right) K_{1,-1} \\
& +\left(c_{i} B_{0}+b_{i} B_{1}+a_{i} B_{2}\right) K_{1,0} \\
& +\left(d_{i} B_{0}+c_{i} B_{1}+b_{i} B_{2}\right) K_{1,1} \\
& +\left(d_{i} B_{1}+c_{i} \beta_{2}\right) K_{1,2}+d_{i} \beta_{2} K_{1,3} \\
& I_{2, i}^{(1)}=a_{i} \delta_{0} K_{2,-2}+\left(b_{i} \delta_{0}+a_{i} \delta_{1}\right) K_{2,-1} \\
& +\left(c_{i} \delta_{0}+b_{i} \delta_{1}+a_{i} \delta_{2}\right) k_{2,0} \\
& +\left(d_{i} \delta_{0}+c_{i} \delta_{1}+b_{i} \delta_{2}\right) k_{2,1} \\
& +\left(d_{i} \delta_{1}+c_{i} \delta_{2}\right) k_{2,2}+d_{i} \delta_{2} K_{2,3} \\
& I_{i}^{(2)}=a_{i}\left(\frac{1}{x_{i-1}}-\frac{1}{x_{i}}\right)+b_{i} \ln \left(\frac{x_{i}}{x_{i-1}}\right)+c_{i}\left(x_{i}-x_{i-1}\right)+t_{2}^{\frac{1}{2} d}\left(x_{i}^{2}-x_{i-1}^{2}\right)
\end{aligned}
$$




$$
\begin{aligned}
& I_{1, i}^{(3)}=a_{i} J_{1,-2}+b_{i} J_{1,-1}+c_{i} J_{1,0}+d_{i} J_{1,1} \\
& I_{2, i}^{(3)}=a_{i} J_{2,-2}+b_{i} J_{2,-1}+c_{i} J_{2,0}+d_{i} J_{2,1} \\
& I_{1, i}^{(4)}=a_{i} J_{1,-1}+b_{i} J_{1,0}+c_{j} J_{1,1}+d_{i} J_{1,2} \\
& I_{2, i}^{(4)}=a_{i} J_{2,-1}+b_{i} J_{2,0}+c_{i} J_{2,1}+d_{i} J_{2,2} \\
& I_{1}^{(5)}=\left[a_{i}+2 \lambda b_{i}+(2 \lambda)^{2} c_{i}+(2 \lambda)^{3} d_{i}\right] L \\
& -\left[b_{i}+2 \lambda c_{i}+(2 \lambda)^{2} d_{i}\right]\left(J_{1,0}-J_{2,0}\right) \\
& -\left[c_{i}+2 \lambda d_{i}\right]\left(J_{1,1}-J_{2,1}\right) \\
& -d_{j}\left(J_{1,2}-J_{2,2}\right) .
\end{aligned}
$$

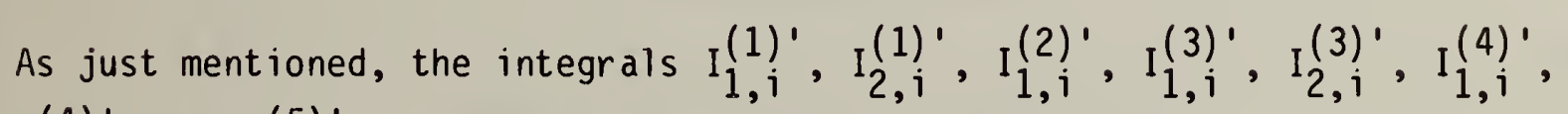
$I_{2, i}^{(4)^{\prime}}$, and $I_{i}^{(5)^{\prime}}$ follow from the above expressions by substituting throughout the corresponding parameters with primes:

$$
\left\{\beta_{0}, \beta_{1}, \ldots\right\} \rightarrow\left\{\beta_{0}{ }^{\prime}, \beta_{1}{ }^{\prime}, \ldots\right\}
$$

and

$$
\left\{a_{i}, b_{i}, c_{i}, d_{i}\right\} \rightarrow\left\{a_{i}^{\prime}, b_{i}^{\prime}, c_{i}^{\prime}, d_{i}^{\prime}\right\}
$$

Finally, we give the explicit expressions for the parameters which appear in the integral in terms of the input variables for the analysis, viz., $\left|\underline{p}_{1}\right|$, $\left|\underline{p}_{2}\right|, \theta, m, M$, and $M^{\star}$, as well as the expressions for the integrals $J_{1, m}$, 
$J_{2, m}, K_{1, m}, K_{2, m}$, and $L$ in terms of these variables. Again we leave the details of the evaluation to the Appendix, giving here only the final results. It is to be noted that in each case, the arqument of the logarithm has been written so that there are no cancellations - all terms in the argument are positive.

For ease of writing we now write $p_{1}$ and $p_{2}$ for $\left|\underline{p}_{1}\right|$ and $\left|\underline{p}_{2}\right|$, respectively. Then with

$$
\begin{aligned}
& \varepsilon_{1}=\sqrt{p_{1}^{2}+m^{2}} \\
& \varepsilon_{2}=\sqrt{p_{2}^{2}+m^{2}} \\
& \mu=\frac{M^{\star 2}-M^{2}}{2 M}
\end{aligned}
$$

we define

$$
\begin{aligned}
\lambda & =\varepsilon_{1} \varepsilon_{2}-p_{1} p_{2} \cos \theta-m^{2} \\
|\underline{u}| & =\left(p_{1}{ }^{2}-2 p_{1} p_{2} \cos \theta+p_{2}{ }^{2}\right)^{1 / 2} \\
\kappa & =\varepsilon_{1}-\varepsilon_{2}-\mu-\frac{\lambda}{M} \\
x_{m} & =\frac{2\left[\lambda+\left(\varepsilon_{1}-\varepsilon_{2}-\mu\right)\left(\varepsilon_{1}-\varepsilon_{2}-|\underline{u}|\right)\right]}{1+\frac{\varepsilon_{1}-\varepsilon_{2}-|\underline{u}|}{M}} \\
x_{M} & =\frac{2\left[\lambda+\left(\varepsilon_{1}-\varepsilon_{2}-\mu\right)\left(\varepsilon_{1}-\varepsilon_{2}+|\underline{u}|\right)\right]}{1+\frac{\varepsilon_{1}-\varepsilon_{2}+|\underline{u}|}{M}}
\end{aligned}
$$




$$
\begin{aligned}
A_{1}= & \left(p_{2}+\frac{\varepsilon_{1} p_{2}-\varepsilon_{2} p_{1} \cos \theta}{M}\right)^{2}+\left(\frac{m p_{1} \sin \theta}{M}\right)^{2} \\
B_{1}= & 2 p_{1}\left[p_{1} p_{2}-\left(\varepsilon_{1} \varepsilon_{2}-m^{2}\right) \cos \theta\right]\left[p_{2}+\frac{\left.\varepsilon_{1} p_{2}-\varepsilon_{2} p_{1} \cos \theta\right]}{M}\right]+\frac{2 m^{2} p_{1}{ }^{2}\left(\varepsilon_{1}-\varepsilon_{2}\right) \sin ^{2} \theta}{M} \\
& -2 \mu\left[p_{2}\left(\varepsilon_{1} p_{2}-\varepsilon_{2} p_{1} \cos \theta\right)+\frac{\lambda\left(\lambda+2 m^{2}\right)}{M}\right] \\
C_{1}= & 4\left[p_{1} \lambda-\mu\left(p_{1} \varepsilon_{2}-\varepsilon_{1} p_{2} \cos \theta\right)\right]^{2}+4\left(\mu m p_{2} \sin \theta\right)^{2} \\
x_{1}= & \frac{B_{1}}{A_{1}} \\
A_{2}= & \left(p_{1}-\frac{\left(\varepsilon_{2} p_{1}-\varepsilon_{1} p_{2} \cos \theta\right)}{M}\right)^{2}+\left(\frac{m p_{2} \sin \theta}{M}\right)^{2} \\
x_{2}= & \frac{B_{2}}{A_{2}} \\
C_{2}= & 4\left[p_{2} \lambda+\mu\left(p_{2} \varepsilon_{1}-\varepsilon_{2} p_{1} \cos \theta\right)\right]^{2}+4\left(\mu m p_{1} \sin \theta\right)^{2} \\
B_{2}= & 2 p_{2}\left[p_{1} p_{2}-\left(\varepsilon_{1} \varepsilon_{2}-m^{2}\right) \cos \theta\right]\left[p_{1}-\frac{\left(\varepsilon_{2} p_{1}-\varepsilon_{1} p_{2} \cos \theta\right)}{M}\right]+\frac{2 m^{2} p_{2}{ }^{2}\left(\varepsilon_{1}-\varepsilon_{2}\right) \sin { }^{2} \theta}{M} \\
& +2 \mu\left[p_{1}\left(\varepsilon_{2} p_{1}-\varepsilon_{1} p_{2} \cos \theta\right)+\frac{\lambda\left(\lambda+2 m^{2}\right)}{M}\right]
\end{aligned}
$$

$$
\zeta=2 m p_{1} p_{2} k \sin \theta
$$$$
n_{1}=\frac{\zeta}{A_{1}}
$$$$
n_{2}=\frac{\zeta}{A_{2}}
$$$$
D_{1}(x)=A_{1}\left[\left(x-x_{1}\right)^{2}+\eta_{1}^{2}\right]=A_{1} x^{2}-2 B_{1} x+C_{1}
$$$$
D_{2}(x)=A_{2}\left[\left(x-x_{2}\right)^{2}+n_{2}^{2}\right]=A_{2} x^{2}-2 B_{2} x+C_{2}
$$ 


$$
\begin{aligned}
& \beta_{0}=8 m^{2} \varepsilon_{1}\left(\varepsilon_{1}-\mu\right) \lambda\left[p_{1}\left(p_{1}-p_{2} \cos \theta\right)-\left(\varepsilon_{1}+\varepsilon_{2}\right) \mu\right] \\
& \beta_{1}=-2 m^{2}\left[2 \varepsilon_{1}\left(\varepsilon_{1}-\mu\right)\left(p_{1}\left(p_{1}-p_{2} \cos \theta\right)-\left(\varepsilon_{1}+\varepsilon_{2}\right)(k+\mu)\right)\right. \\
& \left.+\lambda\left(p_{1}\left(p_{1}-p_{2} \cos \theta\right)-\left(\varepsilon_{1}+\varepsilon_{2}\right) \mu\right)\left(1+\frac{2 \varepsilon_{1}}{M}\right)\right] \\
& \beta_{2}=m^{2}\left[p_{1}\left(p_{1}-p_{2} \cos \theta\right)-\left(\varepsilon_{1}+\varepsilon_{2}\right)(k+\mu)\right]\left(1+\frac{2 \varepsilon_{1}}{M}\right) \\
& \delta_{0}=-8 m^{2} \varepsilon_{2}\left(\varepsilon_{2}+\mu\right) \lambda\left[p_{2}\left(p_{2}-p_{1} \cos \theta\right)+\left(\varepsilon_{1}+\varepsilon_{2}\right) \mu\right] \\
& \delta_{1}=2 m^{2}\left[2 \varepsilon_{2}\left(\varepsilon_{2}+\mu\right)\left(p_{2}\left(p_{2}-p_{1} \cos \theta\right)+\left(\varepsilon_{1}+\varepsilon_{2}\right)(k+\mu)\right)\right. \\
& \left.+\lambda\left(p_{2}\left(p_{2}-p_{1} \cos \theta\right)+\left(\varepsilon_{1}+\varepsilon_{2}\right) \mu\right)\left(1-\frac{2 \varepsilon_{2}}{M}\right)\right] \\
& \delta_{2}=-m^{2}\left[p_{2}\left(p_{2}-p_{1} \cos \theta\right)+\left(\varepsilon_{1}+\varepsilon_{2}\right)(k+\mu)\right]\left(1-\frac{2 \varepsilon_{2}}{M}\right) \\
& \tau_{1}=\frac{1}{\lambda}\left[\lambda^{2}-4 m^{2} \varepsilon_{1} \varepsilon_{2}-2 \mu m^{2}\left(\varepsilon_{1}-\varepsilon_{2}-\mu\right)-2 \mu \varepsilon_{2} \lambda\right] \\
& \tau_{2}=\frac{1}{\lambda}\left[\lambda^{2}-4 m^{2} \varepsilon_{1} \varepsilon_{2}-2 \mu m^{2}\left(\varepsilon_{1}-\varepsilon_{2}-\mu\right)+2 \mu \varepsilon_{1} \lambda\right] \\
& \rho_{1}=\frac{1}{\lambda^{2}}\left[\begin{array}{l}
\left(\lambda+m^{2}\right)\left(2 \varepsilon_{1} \varepsilon_{2}-\lambda\right)+\lambda \kappa^{2}+\left(\lambda+m^{2}\right)((\lambda / M)+\mu) \kappa \\
+\left(m^{2} / M^{2}\right) \lambda^{2}+\frac{1}{2} \lambda^{2}+\left(\lambda^{2} \varepsilon_{1} / M\right)
\end{array}\right] \\
& \rho_{2}=\frac{1}{\lambda^{2}}\left[\begin{array}{l}
\left(\lambda+m^{2}\right)\left(2 \varepsilon_{1} \varepsilon_{2}-\lambda\right)+\lambda \kappa^{2}+\left(\lambda+m^{2}\right)((\lambda / M)+\mu) \kappa \\
+\left(m^{2} / M^{2}\right) \lambda^{2}+\frac{2}{2} \lambda^{2}-\left(\lambda^{2} \varepsilon_{2} / M\right)
\end{array}\right] \\
& \gamma=\frac{1}{\lambda^{2}}\left[\left(\lambda+m^{2}\right)\left(2 \varepsilon_{1} \varepsilon_{2}-\lambda\right)+\lambda k^{2}+\left(\lambda+m^{2}\right)((\lambda / M)+\mu) \kappa\right] \\
& n=1
\end{aligned}
$$




$$
\begin{aligned}
& \beta_{0}^{\prime}=-8 m^{4} \lambda\left[p_{1}\left(p_{1}-p_{2} \cos \theta\right)-\left(\varepsilon_{1}+\varepsilon_{2}\right) \mu\right] \\
& \beta_{1}^{\prime}=4 m^{2}\left[m^{2}\left(p_{1}\left(p_{1}-p_{2} \cos \theta\right)-\left(\varepsilon_{1}+\varepsilon_{2}\right)(k+\mu)\right)+\lambda\left(p_{1}\left(p_{2}-p_{2} \cos \theta\right)-\left(\varepsilon_{1}+\varepsilon_{2}\right) \mu\right)\right] \\
& \beta_{2}^{\prime}=-2 m^{2}\left[p_{1}\left(p_{1}-p_{2} \cos \theta\right)-\left(\varepsilon_{1}+\varepsilon_{2}\right)(k+\mu)\right] \\
& \delta_{0}^{\prime}=8 m^{4} \lambda\left[p_{2}\left(p_{2}-p_{1} \cos \theta\right)+\left(\varepsilon_{1}+\varepsilon_{2}\right) \mu\right] \\
& \delta_{1}^{\prime}=-4 m^{2}\left[m^{2}\left(p_{2}\left(p_{2}-p_{1} \cos \theta\right)+\left(\varepsilon_{1}+\varepsilon_{2}\right)(k+\mu)\right)+\lambda\left(p_{2}\left(p_{2}-p_{1} \cos \theta\right)+\left(\varepsilon_{1}+\varepsilon_{2}\right) \mu\right)\right] \\
& \delta_{2}^{\prime}=2 m^{2}\left[p_{2}\left(p_{2}-p_{1} \cos \theta\right)+\left(\varepsilon_{1}+\varepsilon_{2}\right)(k+\mu)\right] \\
& \tau_{1}^{\prime}=\tau_{2}^{\prime}=-2 \frac{\left[\lambda^{2}-2 m^{2}\left(\lambda+m^{2}\right)\right]}{\lambda} \\
& \rho_{1}^{\prime}=\rho_{2}^{\prime}=\frac{\left(\lambda^{2}-2 m^{4}\right)}{\lambda^{2}} \\
& \gamma^{\prime}=\frac{2\left(\lambda^{2}-m^{4}\right)}{\lambda^{2}} \\
& \eta^{\prime}=-2 .
\end{aligned}
$$

The important integrals are then

$$
\begin{aligned}
J_{1,0} & =\frac{1}{\sqrt{A_{1}}} \operatorname{sgn}\left(A_{1} x_{i}-B_{1}\right) \ln \left(\frac{\left|A_{1} x_{i}-B_{1}\right|+D_{1}^{\frac{2}{2}}\left(x_{j}\right) \sqrt{A_{1}}}{\zeta}\right) \\
& -\frac{1}{\sqrt{A_{1}}} \operatorname{sgn}\left(A_{1} x_{i-1}-B_{1}\right) \ln \left(\frac{\left|A_{1} x_{i-1}-B_{1}\right|+D_{1}^{\frac{2}{2}}\left(x_{i-1}\right) \sqrt{A_{1}}}{\zeta}\right)
\end{aligned}
$$




$$
\begin{aligned}
J_{1,-1} & =\frac{1}{\sqrt{C_{1}}} \operatorname{san}\left(B_{1} x_{i}-C_{1}\right) \ln \left(\frac{\left|B_{1} x_{i}-C_{1}\right|+D_{1}^{\frac{1}{2}}\left(x_{i}\right) \sqrt{C_{1}}}{x_{i} \zeta}\right) \\
& -\frac{1}{\sqrt{C_{1}}} \operatorname{sgn}\left(B_{1} x_{i-1}-C_{1}\right) \ln \left(\frac{\left|B_{1} x_{i-1}-C_{1}\right|+D_{1}^{\frac{1}{2}}\left(x_{i-1}\right) \sqrt{C_{1}}}{x_{i-1} \zeta}\right)
\end{aligned}
$$

where

$$
\operatorname{sgn}(x)=\left\{\begin{array}{rl}
1 & x>0 \\
0 & x=0 \\
-1 & x<0
\end{array} .\right.
$$

Then from $\mathrm{J}_{1,0}$ and $\mathrm{J}_{1,-1}$ we have

$$
\begin{aligned}
& J_{1,1}=\frac{1}{A_{1}}\left[D_{1}^{\frac{2}{2}}\left(x_{i}\right)-D_{1}^{\frac{3}{2}}\left(x_{i-1}\right)\right]+\frac{B_{1}}{A_{1}} J_{1,0} \\
& J_{1,2}=\frac{1}{2 A_{1}}\left[x_{i} D_{1}^{\frac{3}{2}}\left(x_{i}\right)-x_{i-1} D_{1}^{\frac{1}{2}}\left(x_{i-1}\right)\right]+\frac{3 B_{1}}{2 A_{1}} J_{1,1}-\frac{C_{1}}{2 A_{1}} J_{1,0} \\
& J_{1,-2}=-\frac{1}{C_{1}}\left[\frac{D_{1}^{\frac{3}{2}}\left(x_{i}\right)}{x_{i}}-\frac{D_{1}^{\frac{3}{2}}\left(x_{i-1}\right)}{x_{i-1}}\right]+\frac{B_{1}}{C_{1}} J_{1,-1}
\end{aligned}
$$

Then the functions $\mathrm{K}_{1, \mathrm{~m}}$ are given by

$$
\begin{aligned}
& K_{1,0}=\frac{1}{\zeta^{2}}\left[\frac{\left(A_{1} x_{i}-B_{1}\right)}{D_{1}^{\frac{3}{2}}\left(x_{i}\right)}-\frac{\left(A_{1} x_{i-1}-B_{1}\right)}{D_{1}^{\frac{1}{2}}\left(x_{i-1}\right)}\right] \\
& K_{1,1}=-\frac{1}{\zeta^{2}}\left[\frac{\left(-B_{1} x_{i}+C_{1}\right)}{D_{1}^{\frac{3}{2}}\left(x_{i}\right)}-\frac{\left(-B_{1} x_{i-1}+C_{1}\right)}{D_{1}^{\frac{1}{2}}\left(x_{i-1}\right)}\right]
\end{aligned}
$$




$$
\begin{aligned}
& K_{1,2}=\frac{1}{A_{1}}\left[J_{1,0}+2 B_{1} K_{1,1}-C_{1} K_{1,0}\right] \\
& K_{1,3}=\frac{1}{A_{1}}\left[\frac{x_{i}^{2}}{D_{1}^{\frac{1}{2}}\left(x_{i}\right)}-\frac{x_{i-1}{ }^{\frac{1}{2}}\left(x_{i-1}\right)}{D_{1}}\right]+\frac{3 B_{1}}{A_{1}} K_{1,2}-\frac{2 C_{1}}{A_{1}} K_{1,1} \\
& K_{1,-1}=\frac{1}{C_{1}}\left[\frac{1}{D_{1}^{\frac{1}{2}}\left(x_{i}\right)}-\frac{1}{D_{1}^{\frac{1}{2}}\left(x_{i-1}\right)}\right]+\frac{1}{C_{1}} J_{1,-1}+\frac{B_{1}}{C_{1}} K_{1,0} \\
& K_{1,-2}=-\frac{1}{C_{1}}\left[\frac{1}{x_{i} D_{1}^{\frac{1}{2}}\left(x_{i}\right)}-\frac{1}{x_{i-1} D_{1}^{\frac{1}{2}}\left(x_{i-1}\right)}\right]+\frac{3 B_{1}}{C_{1}} K_{1,-1}-\frac{2 A_{1}}{C_{1}} K_{1,0} .
\end{aligned}
$$

The integrals $\mathrm{J}_{2, \mathrm{~m}}$ and $\mathrm{K}_{2, \mathrm{~m}}$ can all be obtained from $\mathrm{J}_{1, m}$ and $\mathrm{K}_{2, \mathrm{~m}}$ merely by replacing all subscripts 1 by the subscript 2 in the expressions above.

Finally, we have the integral L. In order to write this without cancellations, we define ${ }^{7}$

$$
\beta \equiv D_{1}^{\frac{1}{2}}(2 \lambda)=D_{2}^{\frac{1}{2}}(2 \lambda)=2 k \sqrt{\lambda\left(\lambda+2 m^{2}\right)}
$$

and

$$
\begin{gathered}
\xi_{1, i}=\left(2 \lambda A_{1}-B_{1}\right) x_{i}-2 \lambda B_{1}+C_{1} \\
\xi_{2, i}=\left(2 \lambda A_{2}-B_{2}\right) x_{i}-2 \lambda B_{2}+C_{2} \\
\xi_{1, i-1}=\left(2 \lambda A_{1}-B_{1}\right) x_{i-1}-2 \lambda B_{1}+C_{1} \\
\xi_{2, i-1}=\left(2 \lambda A_{2}-B_{2}\right) x_{i-1}-2 \lambda B_{2}+C_{2}
\end{gathered}
$$

${ }^{7}$ See pages 51 and 52 of the appendix, leading to eq. (A.59).

29 
Then

$$
\begin{aligned}
L & =\frac{1}{B} \operatorname{sgn}\left(\xi_{1, j}\right) \ln \left(\frac{\left|\xi_{1, i}\right|+B D_{1}^{\frac{1}{2}}\left(x_{i}\right)}{\zeta}\right) \\
& -\frac{1}{B} \operatorname{sgn}\left(\xi_{1, i-1}\right) \ln \left(\frac{\left|\xi_{1, i-1}\right|+B D_{1}^{\frac{1}{2}}\left(x_{i-1}\right)}{\zeta}\right) \\
& -\frac{1}{B} \operatorname{sgn}\left(\xi_{2, i}\right) \ln \left(\frac{\left|\xi_{2, j}\right|+B D_{2}^{\frac{1}{2}}\left(x_{j}\right)}{\zeta}\right) \\
& +\frac{1}{B} \operatorname{sgn}\left(\xi_{2, i-1}\right) \ln \left(\frac{\left|\xi_{2, i-1}\right|+B D_{2}^{\frac{1}{2}}\left(x_{i-1}\right)}{\zeta}\right) \\
& -\frac{1}{B}\left(\operatorname{sgn}\left(\xi_{1, i}\right)-\operatorname{sgn}\left(\xi_{2, i}\right)\right) \ln \left|x_{i}-2 \lambda\right| \\
& +\frac{1}{B}\left(\operatorname{sgn}\left(\xi_{1, i-1}\right)-\operatorname{sgn}\left(\xi_{2, i-1}\right)\right) \ln \left|x_{i-1}-2 \lambda\right|
\end{aligned}
$$

30 


\section{APPENDIX}

\section{Algebraic Details}

In this appendix we present some of the detailed algebra leading to our final expression for the cross section, in particular to eqs (27), (28), (38), and $(44)-(67)$.

Our starting point is the cross section integrated over the photon azimuthal angle $\varphi_{k}$, given here by eqs (22) and (23). As we have noted, this is the expression given by Mo and Tsai [2], and Tsai [3], and Stein et al. [7], apart from the minor modifications and typographical errors which we have already discussed. Since we perform the remaining integration analytically, the most appropriate variable of integration is $q^{2}$ rather than $\cos \theta_{k}$ in view of the functions $w_{2}\left(-q^{2}\right)$ and $w_{1}\left(-q^{2}\right)$. Further, as we have already noted, the form of the integrand itself is then also somewhat simpler. Referring to eqs (22), (23), and (24), we see that our first task is then to express the quantities listed in (24), as well as the photon energy, $\omega$, in terms of the variable of integration, $q^{2}$, and to derive the differential change of variable given by (25). We see that in (24) the variable $\cos \theta_{k}$ enters only either as $\omega$ or as $\omega \cos \theta_{k}$. (The quantity $b$ enters only as

$$
\begin{aligned}
b^{2} & =p_{2}{ }^{2} \sin ^{2} \theta_{p} \omega^{2} \sin ^{2} \theta_{k} \\
& =p_{2}{ }^{2} \sin ^{2} \theta_{p}\left[\omega^{2}-\left(\omega \cos \theta_{k}\right)^{2}\right]
\end{aligned}
$$

Thus again we have either $\omega$ or $\omega \cos \theta_{k^{\circ}}$ )

Further, we note that the expressions for $\cos \theta_{p}$ and $\cos \theta_{s}$ listed in (24) follow from 


$$
\begin{aligned}
\underline{p}_{1} \cdot \underline{u} & =\underline{p}_{1} \cdot\left(\underline{p}_{1}-\underline{p}_{2}\right) \\
& =p_{1}^{2}-p_{1} p_{2} \cos \theta \\
& =p_{1}|\underline{u}| \cos \theta_{s} \quad\left(p_{1}=\left|\underline{p}_{1}\right|\right)
\end{aligned}
$$

and

$$
\begin{aligned}
\underline{p}_{2} \cdot \underline{u} & =\underline{p}_{2} \cdot\left(\underline{p}_{1}-\underline{p}_{2}\right) \\
& =p_{1} p_{2} \cos \theta-p_{2}^{2} \\
& =p_{2}|\underline{u}| \cos \theta_{p} \quad\left(p_{2}=\left|\underline{p}_{2}\right|\right) .
\end{aligned}
$$

In addition, it is useful to note from $\underline{u}=\underline{p}_{1}-\underline{p}_{2}$ and the figure below,

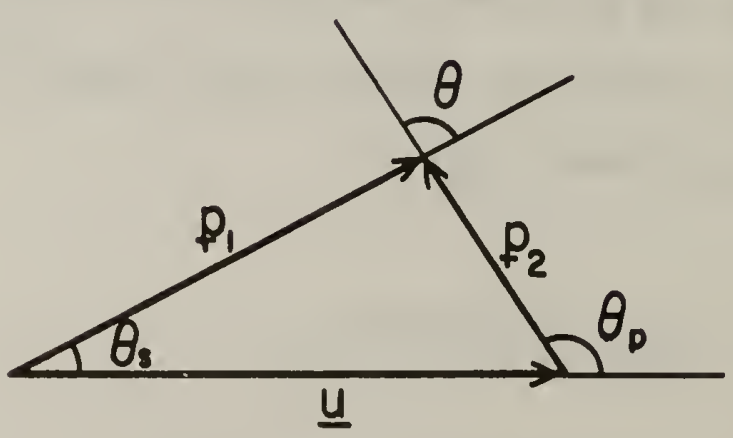

that

$$
\theta_{p}=\theta_{s}+\theta
$$


and

$$
\frac{p_{2}}{\sin \theta_{s}}=\frac{p_{1}}{\sin \theta_{p}}=\frac{|\underline{u}|}{\sin \theta} .
$$

Thus

$$
p_{2} \sin \theta_{p}=p_{1} \sin \theta_{s}
$$

and

$$
\begin{aligned}
& \underline{\mid u} \mid \sin \theta_{p}=p_{2} \sin \theta \\
& \underline{\mid u} \mid \sin \theta_{s}=p_{2} \sin \theta \text {. }
\end{aligned}
$$

The Jacobian expressed by eq (25) follows directly from eqs (11) and (15). From eq (11), viz.,

$$
\omega=\frac{u^{2}-M^{\star 2}}{2\left(u_{0}-\underline{\mid u} \mid \cos \theta_{k}\right)},
$$

we have

$$
\begin{aligned}
d \omega & =\frac{\left(u^{2}-M^{\star 2}\right)|\underline{u}| d\left(\cos \theta_{k}\right)}{2\left(u_{0}-\underline{\left.\underline{u} \mid \cos \theta_{k}\right)^{2}}\right.} \\
& =\frac{\omega|\underline{u}| d\left(\cos \theta_{k}\right)}{\left(u_{0}-\mid \underline{\left.\underline{u} \mid \cos \theta_{k}\right)}\right.},
\end{aligned}
$$


and from eq (15), viz.,

$$
\omega=\varepsilon_{1}-\varepsilon_{2}-\mu+\frac{q^{2}}{2 M} \text {, }
$$

we have

$$
d \omega=\frac{1}{2 M} d\left(q^{2}\right)
$$

so that

$$
\frac{\omega|\underline{u}| d\left(\cos \theta_{k}\right)}{\left(u_{0}-|\underline{u}| \cos \theta_{k}\right)}=\frac{1}{2 M} d\left(q^{2}\right)
$$

or

$$
\frac{2 M \omega d\left(\cos \theta_{k}\right)}{q^{4}\left(u_{0}-|\underline{u}| \cos \theta_{k}\right)}=\frac{1}{|\underline{u}|} \frac{d\left(q^{2}\right)}{q^{4}} \text {, }
$$

which is eq $(25)$.

Next we express $\omega$ and $\omega \cos \theta_{k}$ in terms of $q^{2}$. In eqs (12)-(15) we have shown that

$$
\omega=\varepsilon_{1}-\varepsilon_{2}-\mu+\frac{q^{2}}{2 M} \text {, }
$$

in which the quantities $\varepsilon_{1}, \varepsilon_{2}, \mu$, and $M$ are defined in (4). Next, from eq (6) we have (four-vectors are implied in the next two lines) 


$$
\begin{aligned}
q^{2} & =\left(p_{1}-p_{2}-k\right)^{2} \\
& =\left(p_{1}-p_{2}\right)^{2}-2 k \cdot\left(p_{1}-p_{2}\right) \\
& =-2 \lambda-2 \omega\left(\varepsilon_{1}-\varepsilon_{2}\right)+2 \omega|\underline{u}| \cos \theta_{k}
\end{aligned}
$$

where, as in (45),

$$
\lambda=\varepsilon_{1} \varepsilon_{2}-\underline{p}_{1} \cdot \underline{p}_{2}-m^{2} \text {. }
$$

Thus, using (A.8),

$$
\omega \cos \theta_{k}=\frac{1}{|\underline{u}|}\left[\lambda+\left(\varepsilon_{1}-\varepsilon_{2}\right)\left(\varepsilon_{1}-\varepsilon_{2}-\mu\right)+\frac{1}{2} q^{2}\left(1+\frac{\varepsilon_{1}-\varepsilon_{2}}{M}\right)\right] .
$$

From (A.8) and (A.10) we note that both $\omega$ and $\omega \cos \theta_{k}$ are linear functions of $q^{2}$. Thus, referring to (24), we see that $a$ and $a^{\prime}$ are also linear functions of $q^{2}$, and that $a^{2}-b^{2}$ and $a^{\prime 2}-b^{2}$ are quadratic functions of $q^{2}$.

The algebra involved in our calculation is reduced considerably by noting that upon making the substitutions

$$
\text { subscripts }(1+2,2+1), \mu \rightarrow-\mu, M \rightarrow-M
$$

we have, from (A.8) and (A.10),

$$
\omega \rightarrow-\omega
$$

and

$$
\omega \cos \theta_{k} \rightarrow \omega \cos \theta_{k} \quad \text { (i.e., } \omega \cos \theta_{k} \text { does not change), }
$$


and from (A.1) and (A.2)

$$
\cos \theta_{p}+-\cos \theta_{s} \quad, \quad \cos \theta_{s} \rightarrow-\cos \theta_{p} .
$$

Thus when $a$ and $a^{\prime}$ in (24) are expressed in terms of $q^{2}$ as the sole variable, we may obtain a' from a simply by making the substitutions indicated in (A.11) and introducing an overall minus sign. That is, under (A.11)

$$
a+-a^{\prime} \quad, \quad a^{\prime}+-a \quad \text {. }
$$

In similar fashion we see from (A.5) that $b^{2}$ as defined in (24) remains unchanged under the substitutions of (A.11). Thus under (A.11) we also have

$$
a^{2}-b^{2}+a^{12}-b^{2} .
$$

We thus need only write $a$ and $a^{2}-b^{2}$ in terms of $q^{2}$, and then use (A.11)(A.13) to obtain $a^{\prime}$ and $a^{\prime 2}-b^{2}$. We start then with a as given in (24), viz.,

$$
a=\varepsilon_{2} \omega-p_{2} \cos \theta_{p} \omega \cos \theta_{k}
$$

In this expression we then substitute $\omega$ as given by $(A .8), \cos \theta_{p}$ as given by (24) and $\omega \cos \theta_{k}$ as given by (A.10). After some tedious but straightforward algebra we find

$$
a=\frac{1}{\underline{u}^{2}}\left\{\begin{array}{l}
{\left[p_{1}\left(p_{1}-p_{2} \cos \theta\right)-\left(\varepsilon_{1}+\varepsilon_{2}\right) \mu\right] \lambda} \\
+\frac{1}{2} q^{2}\left[p_{1}\left(p_{1}-p_{2} \cos \theta\right)-\left(\varepsilon_{1}+\varepsilon_{2}\right)(k+\mu)\right]
\end{array}\right\} \text {. }
$$


Then, applying (A.11) and (A.12) we have

$$
a^{\prime}=-\frac{1}{\underline{u}^{2}}\left\{\begin{array}{l}
{\left[p_{2}\left(p_{2}-p_{1} \cos \theta\right)+\left(\varepsilon_{1}+\varepsilon_{2}\right) \mu\right] \lambda} \\
+\frac{1}{2} q^{2}\left[p_{2}\left(p_{2}-p_{1} \cos \theta\right)+\left(\varepsilon_{1}+\varepsilon_{2}\right)(k+\mu)\right]
\end{array}\right\}
$$

where $\lambda$ and $k$ are given by eq (43) in the body of this report, viz.,

$$
\begin{aligned}
& \lambda=\varepsilon_{1} \varepsilon_{2}-p_{1} p_{2} \cos \theta-m^{2} \\
& \kappa=\varepsilon_{1}-\varepsilon_{2}-\mu-\frac{\lambda}{M}
\end{aligned}
$$

We next consider $a^{2}-b^{2}$, which, as we have noted, is a quadratic function of $q^{2}$. The quantity $b$ is given in (24), viz.,

$$
b=-\omega_{2} \sin \theta_{p} \sin \theta_{k}
$$

and from (A.6) this may be written as

$$
b=-\frac{u_{1} p_{2}}{|\underline{u}|} \sin \theta \sin \theta_{k}
$$

from which

$$
b^{2}=\frac{\left(p_{1} p_{2} \sin \theta\right)^{2}}{\underline{u}^{2}}\left[\omega^{2}-\left(\omega \cos \theta_{k}\right)^{2}\right] .
$$

We then substitute in this expression $\omega$ and $\omega \cos \theta_{k}$ as given by (A.8) and (A.10), and obtain $b^{2}$ as a quadratic function of $q^{2}$. With a as given by (A.14) we then have then have, after much tedious but straightforward algebra, the following expression for $a^{2}-b^{2}$ : 


$$
a^{2}-b^{2}=\frac{1}{4 \underline{u}^{2}}\left\{A_{1}\left(q^{2}\right)^{2}+2 B{ }_{1} q^{2}+C_{1}\right\}
$$

where $A_{1}, B_{1}$, and $C_{1}$ are qiven by eqs (47). It should be noted from eqs (47) (in particular for the integrations that follow) that

$$
A_{1}>0, C_{1}>0
$$

With

$$
x=-q^{2}>0
$$

as defined earlier in eq (26), we then write

$$
a^{2}-b^{2}=\frac{1}{4 \underline{u}^{2}} D_{1}(x)
$$

where

$$
\begin{aligned}
D_{1}(x) & =A_{1} x^{2}-2 B_{1} x+C_{1} \\
& =A_{1}\left[\left(x-x_{1}\right)^{2}+n_{1}{ }^{2}\right]
\end{aligned}
$$

with

$$
x_{1}=\frac{B_{1}}{A_{1}}
$$

and 


$$
n_{1}^{2}=\frac{A_{1} C_{1}-B_{1}^{2}}{A_{1}^{2}}
$$

With $A_{1}, B_{1}$, and $C_{1}$ as given by (47) we find, after considerable algebra,

$$
A_{1} C_{1}-B_{1}^{2}=\left(2 m p_{1} p_{2} k \sin \theta\right)^{2} \equiv \zeta^{2}>0
$$

Again making the substitutions (A.11), we obtain

$$
a^{\prime 2}-b^{2}=\frac{1}{4 \underline{u}^{2}}\left\{A_{2}\left(q^{2}\right)^{2}+2 B_{2} q^{2}+C_{2}\right\}
$$

with $A_{2}, B_{2}$, and $C_{2}$ as given by eqs (48). We now write

$$
a^{12}-b^{2}=\frac{1}{4 \underline{u}^{2}} D_{2}(x)
$$

where

$$
\begin{aligned}
D_{2}(x) & =A_{2} x^{2}-2 B_{2} x+C_{2} \\
& =A_{2}\left[\left(x-x_{2}\right)^{2}+\eta_{2}{ }^{2}\right]
\end{aligned}
$$

with

$$
x_{2}=\frac{B_{2}}{A_{2}}
$$

and 


$$
n_{2}{ }^{2}=\frac{A_{2} C_{2}-B_{2}{ }^{2}}{A_{2}{ }^{2}} .
$$

From ( $A .23$ ) we see that $A_{1} C_{1}-B_{1}{ }^{2}$ is unchanged under the substitutions (A.11). Thus

$$
A_{2} C_{2}-B_{2}^{2}=A_{1} C_{1}-B_{1}^{2}=\zeta^{2} \text {. }
$$

Next we consider the terms in (23) with factor $v$. From eqs (24), (A.14), and (A.15) we have

$$
\begin{aligned}
v & =\frac{1}{a^{\prime}-a} \\
& =\frac{-\underline{u}^{2}}{\left(\frac{1}{2} q^{2}+\lambda\right)\left[p_{2}\left(p_{2}-p_{1} \cos \theta\right)+p_{1}\left(p_{1}-p_{2} \cos \theta\right)\right]} \\
& =\frac{-2}{q^{2}+2 \lambda}
\end{aligned}
$$

since, from eq (8b),

$$
\underline{u}^{2}=p_{1}^{2}-2 p_{1} p_{2} \cos \theta+p_{2}^{2} \text {. }
$$

Thus the terms with factor $v$ in the integral (22) have an overall factor

$$
\frac{1}{q^{4}\left(q^{2}+2 \lambda\right)}
$$


As may be seen by referring to these terms in (23), they also have a coefficient of the form $c_{0}+c_{1} q^{2}+c_{2}\left(q^{2}\right)^{2}$. (For the terms multiplying $W_{1}\left(-q^{2}\right)$ we have $c_{1}=c_{2}=0$.) Then, to obtain our expression (28), we write

$$
\begin{aligned}
\frac{c_{0}+c_{1} q^{2}+c_{2}\left(q^{2}\right)^{2}}{q^{4}\left(q^{2}+2 \lambda\right)} & =\left(\frac{c_{0}-2 \lambda c_{1}+(2 \lambda)^{2} c_{2}}{(2 \lambda)^{2}}\right) \frac{1}{\left(q^{2}+2 \lambda\right)} \\
& -\left(\frac{c_{0}-2 \lambda c_{1}}{(2 \lambda)^{2}}\right) \frac{1}{q^{2}}+\left(\frac{c_{0}}{2 \lambda}\right) \frac{1}{\left(q^{2}\right)^{2}} .
\end{aligned}
$$

Finally we substitute (A.14), (A.15), (A.19), (A.25), and (A.30) with $x=-q^{2}$, in the cross section given by (22) and (23), using (A.31) for the terms with factor $v$. Collecting the terms with similar denominators then gives the expression (28).

We derive next the expression given in (46) for the limits of integration, $x_{m}$ and $x_{M^{*}}$ From (11), viz.,

$$
\omega=\frac{u^{2}-M^{\star 2}}{2\left(u_{0}-|\underline{u}| \cos \theta_{k}\right)}
$$

we see that $\omega$ is an increasing function of $\cos \theta_{k}$, and from (15), viz.,

$$
\omega=\varepsilon_{1}-\varepsilon_{2}-\mu+\frac{q^{2}}{2 M} \text {. }
$$

it follows that $q^{2}$ is also an increasing function of $\cos \theta_{k}$, and hence that $x=-q^{2}$ is a decreasing function of $\cos \theta_{k}$. Thus the minimum value of $x$, viz.,

$$
x=x_{m}=-q_{m}^{2} \quad \text { corresponds to } \cos \theta_{k}=+1
$$


and the maximum value of $x$, viz.,

$$
x=x_{M}=-q_{M}^{2} \quad \text { corresponds to } \cos \theta_{k}=-1 \text {. }
$$

We now compare eq (15) given just above with eq (A.10), viz.,

$$
\omega \cos \theta_{k}=\frac{1}{|\underline{u}|}\left[\lambda+\left(\varepsilon_{1}-\varepsilon_{2}\right)\left(\varepsilon_{1}-\varepsilon_{2}-\mu\right)+\frac{\varepsilon_{1}-\varepsilon_{2}}{2} q^{2}\left(1+\frac{\underline{M}}{M}\right) .\right.
$$

When $\cos \theta_{k}=+1$ these two equations give

$$
\varepsilon_{1}-\varepsilon_{2}-\mu+\frac{q_{m}^{2}}{2 M}=\frac{1}{|\underline{u}|}\left[\lambda+\left(\varepsilon_{1}-\varepsilon_{2}\right)\left(\varepsilon_{1}-\varepsilon_{2}-\mu\right)+\frac{1}{2} q_{m}{ }^{2}\left(1+\frac{\varepsilon_{1}-\varepsilon_{2}}{M}\right)\right]
$$

or

$$
x_{m}=\frac{2\left[\lambda+\left(\varepsilon_{1}-\varepsilon_{2}-|\underline{u}|\right)\left(\varepsilon_{1}-\varepsilon_{2}-\mu\right)\right]}{1+\frac{\varepsilon_{1}-\varepsilon_{2}-|\underline{u}|}{M}}
$$

as in (46). When $\cos \theta_{k}=-1$ these two equations give

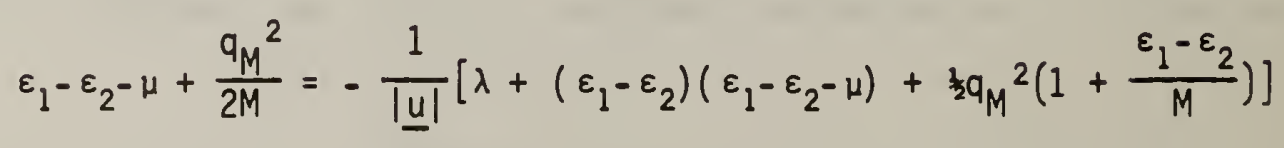

or

$$
x_{M}=\frac{2\left[\lambda+\left(\varepsilon_{1}-\varepsilon_{2}+|\underline{u}|\right)\left(\varepsilon_{1}-\varepsilon_{2}-\mu\right)\right]}{1+\frac{\varepsilon_{1}-\varepsilon_{2}+|\underline{u}|}{M}}
$$


We next show that the point $x=-q^{2}=2 \lambda$, where, as in (45),

$$
\lambda=\varepsilon_{1} \varepsilon_{2}-p_{1} p_{2} \cos \theta-m^{2},
$$

lies within the range of integration:

$$
x_{m}<2 \lambda<x_{M}
$$

as noted in (29). Now from (A.9) we see that $x=-q^{2}=2 \lambda$ implies

$$
\cos \theta_{k}=\frac{\varepsilon_{1}-\varepsilon_{2}}{|\underline{u}|}
$$

Now as we show immediately below,

$$
0<\frac{\varepsilon_{1}-\varepsilon_{2}}{|\underline{u}|}<1
$$

Thus since the integration region, when we choose $\cos \theta_{k}$ as integration variable, is the entire range of possible values, $-1<\cos \theta_{k}<1$, we see from (A.32) and (A.33) that the point $x=2 \lambda$ corresponds to a point in the range of integration with $x$ as integration variable (recall that $x$ is a uniformly decreasing function of $\cos \theta_{k}$ ), i.e., that

$$
x_{m}<2 \lambda<x_{M}
$$

Further, since the photon energy, given by (15), must be positive for $\cos \theta_{k}$ within the range of integration, i.e., 


$$
\omega=\varepsilon_{1}-\varepsilon_{2}-\mu+\frac{q^{2}}{2 M}>0 \text {, }
$$

it follows that this is also true for the point $-q^{2}=2 \lambda$ just considered. Thus

$$
\kappa=\varepsilon_{1}-\varepsilon_{2}-\mu-\frac{\lambda}{M}>0 \text {. }
$$

Returning now to the proof of (A.33), we note from $\varepsilon_{2}<\varepsilon_{1}$ (and $p_{2}<p_{1}$ ) that

$$
\varepsilon_{1}-p_{1}=\frac{m^{2}}{\varepsilon_{1}+p_{1}}<\frac{m^{2}}{\varepsilon_{2}+p_{2}}=\varepsilon_{2}-p_{2} .
$$

Thus

$$
0<\varepsilon_{1}-\varepsilon_{2}<p_{1}-p_{2}<\left|\underline{p}_{1}-\underline{p}_{2}\right|=|\underline{u}| \text {. }
$$

from which (A.33) follows.

Finally, we conclude our presentation of algebraic details with a consideration of the expressions given in eqs (53)-(67) for the integrals defined in eqs (39)-(41). We begin with $J_{1,0}$, defined by eq (39), viz.,

$$
J_{1,0}=\int_{x_{i-1}}^{x_{i}} \frac{d x}{D_{1}^{1 / 2}(x)}
$$

where from eq (50a)

$$
D_{1}(x)=A_{1} x^{2}-2 B_{1} x+C_{1}
$$


A straighforward integration gives [9]

$$
\begin{aligned}
J_{1,0} & =\frac{1}{\sqrt{A_{1}}} \ln \left(\frac{A_{1} x_{i}-B_{1}+D_{1}^{\frac{1}{2}}\left(x_{i}\right) \sqrt{A_{1}}}{c_{0}}\right) \\
& -\frac{1}{\sqrt{A_{1}}} \ln \left(\frac{A_{1} x_{i-1}-B_{1}+D_{1}^{\frac{1}{2}}\left(x_{i-1}\right) \sqrt{A_{1}}}{c_{0}}\right)
\end{aligned}
$$

where $c_{0}$ is an arbitrary constant. Now for reasons that will be apparent momentarily, we choose

$$
\begin{aligned}
c_{0} & =\sqrt{A_{1} C_{1}-B_{1}{ }^{2}} \\
& \equiv \zeta=2 m p_{1} p_{2} k \sin \theta>0
\end{aligned}
$$

from (A.23) and (A.34). The arguments of the logarithms in (A.36) are then dimensionless and, from eqs. (47) and ( $A .23)$, are of order $\varepsilon / m$ if $A_{1} x-B_{1}>0$ (where $x=x_{i}$ or $x_{i-1}$ ). On the other hand, since

$$
\begin{aligned}
D_{1}(x) A_{1}-\left(A_{1} x-B_{1}\right)^{2} & =\left(A_{1} x^{2}-2 B_{1} x+C_{1}\right) A_{1}-\left(A_{1} x-B_{1}\right)^{2} \\
& =A_{1} C_{1}-B_{1}{ }^{2}
\end{aligned}
$$

we have

$$
\frac{A_{1} x-B_{1}+D_{1}^{\frac{1}{2}}(x) \sqrt{A_{1}}}{\zeta}=\frac{\zeta}{-\left(A_{1} x-B_{1}\right)+D_{1}^{\frac{1}{2}}(x) \sqrt{A_{1}}}
$$


Thus if $A_{1} x-B_{1}<0$ we can write the arguments of the logarithms in (A.36) in the form given by the right hand side of (A.39), from which we see that the argument is then of order $m / \varepsilon$. Thus if $A_{1} x-B_{1}<0$ there is a cancellation, leaving terms of relative order $(m / \varepsilon)^{2}$. We will therefore write the argument so that there is no cancellation, i.e., as given by the left hand side of (A.39) if $A_{1} x-B_{1}>0$ and by the right hand side of (A.39) if $A_{1} x-B_{1}<0$. These can be combined into a single expression by writing

$$
\begin{aligned}
J_{1,0} & =\frac{1}{\sqrt{A_{1}}} \operatorname{sgn}\left(A_{1} x_{i}-B_{1}\right) \ln \left(\frac{\left|A_{1} x_{i}-B_{1}\right|+D_{1}^{\frac{1}{2}}\left(x_{i}\right) \sqrt{A_{1}}}{\zeta}\right) \\
& -\frac{1}{\sqrt{A_{1}}} \operatorname{sgn}\left(A_{1} x_{i-1}-B_{1}\right) \ln \left(\frac{\left|A_{1} x_{i-1}-B_{1}\right|+D_{1}^{\frac{1}{2}}\left(x_{i-1}\right) \sqrt{A_{1}}}{\zeta}\right)
\end{aligned}
$$

where

$$
\operatorname{sgn}(x)=\left\{\begin{array}{cl}
1 & x>0 \\
0 & x=0 \\
-1 & x<0
\end{array} .\right.
$$

This is the result given in (53).

The integral $J_{1,-1}$ is also defined by (39), viz.,

$$
J_{1,-1}=\int_{x_{i-1}}^{x_{i}} \frac{d x}{x_{1}^{\frac{1}{2}}(x)}
$$

Again, a straightforward integration gives [9] 


$$
\begin{aligned}
J_{1,-1} & =-\frac{1}{\sqrt{C_{1}}} \ln \left(\frac{-B_{1} x_{i}+C_{1}+D_{1}^{\frac{2}{2}}\left(x_{i}\right) \sqrt{C_{1}}}{x_{i} c_{0}{ }^{\prime}}\right) \\
& +\frac{1}{\sqrt{C_{1}}} \ln \left(\frac{-B_{1} x_{i-1}+C_{1}+D_{1}^{\frac{2}{2}}\left(x_{i-1}\right) \sqrt{c_{1}}}{x_{i-1} c_{0}}\right)
\end{aligned}
$$

where $C_{0}{ }^{\prime}$ is an arbitrary constant. In analogy with (A.38) we note that

$$
\begin{aligned}
D_{1}(x) C_{1}-\left(-B_{1} x+C_{1}\right)^{2} & =\left(A_{1} x^{2}-2 B_{1} x+C_{1}\right) C_{1}-\left(-B_{1} x+C_{1}\right)^{2} \\
& =x^{2}\left(A_{1} C_{1}-B_{1}{ }^{2}\right) \\
& =x^{2} \zeta^{2} .
\end{aligned}
$$

Thus we again choose $c_{0}{ }^{\prime}=\zeta$, and note that

$$
\frac{-B_{1} x+C_{1}+D_{1}^{\frac{1}{2}}(x) \sqrt{C_{1}}}{x \zeta}=\frac{x \zeta}{-\left(-B_{1} x-C_{1}\right)+D_{1}^{\frac{1}{2}}(x) \sqrt{C_{1}}}
$$

Thus if $-B_{1} x+C_{1}<0$ we can write the arguments of the logarithms in (A.41) in the form given by the right hand side of (A.43), thus avoiding the cancellation. As before, we now write $\mathrm{J}_{1,-1}$ in a form which avoids the possible cancellation in the arguments of the logarithms, viz., 


$$
\begin{aligned}
J_{1,-1} & =-\frac{1}{\sqrt{C_{1}}} \operatorname{sgn}\left(-B_{1} x_{i}+C_{1}\right) \ln \left(\frac{\left|-B_{1} x_{i}+C_{1}\right|+D_{1}^{\frac{1}{2}}\left(x_{i}\right) \sqrt{C_{1}}}{x_{i} \zeta}\right) \\
& +\frac{1}{\sqrt{C_{1}}} \operatorname{sgn}\left(-B_{1} x_{i-1}+C_{1}\right) \ln \left(\frac{\left|-B_{1} x_{i-1}+C_{1}\right|+D_{1}^{\frac{1}{2}}\left(x_{i-1}\right) \sqrt{C_{1}}}{x_{i-1} \zeta}\right)
\end{aligned}
$$

which is equivalent to the result given in (54).

The integrals $k_{1,0}$ and $k_{1,1}$, defined by $(40)$ and given explicitely by (59) and (60), are elementary and present no particular difficulty. The

expression of $\mathrm{J}_{1, \mathrm{~m}}$ and $\mathrm{K}_{1, \mathrm{~m}}$ in terms of $\mathrm{J}_{1,0}, \mathrm{~J}_{1,-1}, \mathrm{~K}_{1,0}$, and $\mathrm{K}_{1,1}$, given in (56)-(64), is also elementary and may be found, for example, in the integral tables of Gröbner and Hofreiter [9].

Lastly, we have the integral L, defined in (41), viz.,

$$
L=\int_{x_{i-1}}^{x_{i}} \frac{1}{(2 \lambda-x)}\left(\frac{1}{D_{1}^{1 / 2}(x)}-\frac{1}{D_{2}^{1 / 2}(x)}\right) d x \quad .
$$

We first note that

$$
D_{1}(2 \lambda)=D_{2}(2 \lambda)
$$

and thus the integrand has in fact no singularity at $x=2 \lambda$. This may be seen most easily as follows: From (A.30)

$$
\begin{aligned}
a^{\prime}-a & =-\frac{1}{2}\left(q^{2}+2 \lambda\right) \\
& =\frac{1}{2}(x-2 \lambda),
\end{aligned}
$$


and from (A.19) and (A.25)

$$
D_{1}(x)-D_{2}(x)=4 \underline{u}^{2}\left(a^{2}-a^{\prime 2}\right)
$$

Thus

$$
D_{1}(x)-D_{2}(x)=-2 \underline{u}^{2}\left(a+a^{\prime}\right)(x-2 \lambda)
$$

and

$$
D_{1}(2 \lambda)=D_{2}(2 \lambda)
$$

We define ${ }^{8}$

$$
B \equiv D_{1}^{1 / 2}(2 \lambda)=D_{2}^{1 / 2}(2 \lambda)
$$

In analogy with the procedure followed for $\mathrm{J}_{1,0}$ and $\mathrm{J}_{1,-1}$ we first perform the integration straightforwardly (see [9]) and write

$$
\begin{aligned}
L & =\frac{1}{\beta} \ln \left(\frac{\xi_{1, i}+\beta D_{1}^{\frac{1}{2}}\left(x_{i}\right)}{\left|x_{i}-2 \lambda\right| \zeta}\right) \\
& -\frac{1}{\beta} \ln \left(\frac{\xi_{1, i-1}+\beta D_{1}^{\frac{1}{2}}\left(x_{i-1}\right)}{\left|x_{i-1}-2 \lambda\right| \zeta}\right) \\
& -\frac{1}{\beta} \ln \left(\frac{\xi_{2, j}+\beta D_{2}^{\frac{1}{2}}\left(x_{i}\right)}{\left|x_{i}-2 \lambda\right| \zeta}\right) \\
& +\frac{1}{\beta} \ln \left(\frac{\xi_{2, i-1}+\beta D_{2}^{\frac{1}{2}}\left(x_{i-1}\right)}{\left|x_{i-1}-2 \lambda\right| \zeta}\right)
\end{aligned}
$$

${ }^{8}$ See the end of this appendix for a derivation of the value of $B$. 
where

$$
\begin{gathered}
\xi_{1}(x)=\left(2 \lambda A_{1}-B_{1}\right) x-2 \lambda B_{1}+C_{1} \\
\xi_{2}(x)=\left(2 \lambda A_{2}-B_{2}\right) x-2 \lambda B_{2}+C_{2} \\
\xi_{1, i}=\xi_{1}\left(x_{i}\right), \quad \xi_{1, i-1}=\xi_{1}\left(x_{i-1}\right) \\
\xi_{2, i}=\xi_{2}\left(x_{i}\right), \quad \xi_{2, i-1}=\xi_{2}\left(x_{i-1}\right)
\end{gathered}
$$

Then noting that

$$
\frac{\xi_{1}(x)+B D_{1}^{\frac{1}{2}}(x)}{|x-2 \lambda| \zeta}=\frac{|x-2 \lambda| \zeta}{-\xi_{1}(x)+B D_{1}^{\frac{1}{2}}(x)}
$$

(and a similar equation in which all subscripts 1 are replaced by the subscript 2), we start by writing $L$ in a form similar to that just used for $\mathrm{J}_{1,0}$ and $\mathrm{J}_{1,-1}$, so that there is no cancellation when any of the terms $\xi_{1, i}$, $\xi_{2, i}, \xi_{1, i-1}$, or $\xi_{2, i-1}$ is negative:

$$
\begin{aligned}
L & =\frac{1}{\beta} \operatorname{sgn}\left(\xi_{1, i}\right) \ln \left(\frac{\left|\xi_{1, j}\right|+\beta D_{1}^{\frac{1}{2}}\left(x_{i}\right)}{\left|x_{i}-2 \lambda\right| \zeta}\right) \\
& -\frac{1}{\beta} \operatorname{sgn}\left(\xi_{1, i-1}\right) \ln \left(\frac{\left|\xi_{1, i-1}\right|+B D_{1}^{\frac{1}{2}}\left(x_{i-1}\right)}{\left|x_{i-1}-2 \lambda\right| \zeta}\right) \\
& -\frac{1}{\beta} \operatorname{sgn}\left(\xi_{2, i}\right) \ln \left(\frac{\left|\xi_{2, j}\right|+B D_{2}^{\frac{1}{2}}\left(x_{j}\right)}{\left|x_{j}-2 \lambda\right| \zeta}\right) \\
& +\frac{1}{\beta} \operatorname{sgn}\left(\xi_{2, i-1}\right) \ln \left(\frac{\left|\xi_{2, i-1}\right|+B D_{2}^{\frac{1}{2}}\left(x_{i-1}\right)}{\left|x_{i-1}-2 \lambda\right| \zeta}\right) .
\end{aligned}
$$


However, in this form of the expression for $L$ there can be a large cancellation for $x_{j}$ or $x_{i-1}$ sufficiently close to $2 \lambda$, since, as we have noted, the integrand in the integral for $L,(A .44)$, has no singularity for $x=2 \lambda$. We therefore write $L$ in a form such that this cancellation is manifest. Equation (A.54) may simply be written in the form

$$
L=L_{i}-L_{i-1}
$$

where

$$
\begin{aligned}
L_{i} & =\frac{1}{\beta} \operatorname{sgn}\left(\xi_{1, i}\right) \ln \left(\frac{\left|\xi_{1, j}\right|+\beta D_{1}^{\frac{1}{2}}\left(x_{j}\right)}{\zeta}\right) \\
& -\frac{1}{\beta} \operatorname{sgn}\left(\xi_{2, i}\right) \ln \left(\frac{\left|\xi_{2, j}\right|+\beta D_{2}^{\frac{1}{2}}\left(x_{j}\right)}{\zeta}\right) \\
& -\frac{1}{\beta}\left(\operatorname{sgn}\left(\xi_{1, i}\right)-\operatorname{sgn}\left(\xi_{2, i}\right)\right) \ln \left|x_{i}-2 \lambda\right|
\end{aligned}
$$

and a similar expression with $i$ replaced by $i-1$. This is the result given in eq (67). Now from (A.51) and eqs (50a) and (50b) we have

$$
\begin{aligned}
& \xi_{1}(2 \lambda)=D_{1}(2 \lambda) \\
& \xi_{2}(2 \lambda)=D_{2}(2 \lambda)
\end{aligned}
$$

and hence from (A.48) and eqs (50a) and (50b) 


$$
\xi_{1}(2 \lambda)=\xi_{2}(2 \lambda)>0
$$

Therefore, for $x_{i}$ sufficiently close to $2 \lambda$ we have $\operatorname{sgn}\left(\xi_{1, j}\right)=+1$, $\operatorname{sqn}\left(\xi_{2, i}\right)=+1$ and thus $L_{i}$ in $(A .56)$ can be written more simply as

$$
L_{i}=\frac{1}{\beta} \ln \left(\frac{\left|\xi_{1, j}\right|+B D_{1}^{\frac{1}{2}}\left(x_{j}\right)}{\left|\xi_{2, j}\right|+B D_{2}^{\frac{1}{2}}\left(x_{j}\right)}\right) .
$$

Here (i.e., when $\operatorname{sgn}\left(\xi_{1, j}\right)=\operatorname{sgn}\left(\xi_{2, j}\right)$ ) there is clearly no singularity for $x_{i}=2 \lambda$. These observations can be used to avoid any possible problem arising in a computer program from $x_{i}$ or $x_{i-1}$ being chosen too close to $2 \lambda$. We may simply define $L$ by $(A .55)$, with $L_{i}\left(\right.$ and $L_{i-1}$ ) given by $(A .59)$ if both $\xi_{1, i}>0$ and $\xi_{2, i}>0$, and otherwise given by $(A .56)$. (It $c$ an be shown that we can not have both $\xi_{1, i}<0$ and $\left.\xi_{2, i}<0.\right)$

Finally, we require the value of $B$, defined in (A.49), given in the body of the report in eq (65), and appearing in the equations for $L,(A .50)$ and (A.54)-(A.59). From the definition, (A.49),

$$
B=D_{1}^{\frac{1}{2}}(2 \lambda)=D_{2}^{\frac{1}{2}}(2 \lambda) \text {, }
$$

it is clear that one can evaluate $B$ simply by substituting $x=2 \lambda$ in the equations defining $D_{1}(x)$ and $D_{2}(x),(50 a)$, and $(50 b)$. This is a rather cumbersome procedure, however. We therefore give the following simpler derivation:

For $x=2 \lambda$ we have

$$
\begin{gathered}
a^{\prime}=a \\
D_{1}(2 \lambda)=D_{2}(2 \lambda),
\end{gathered}
$$


from (A.46) and (A.47), and

$$
\omega=k
$$

from eqs (15) and (A.16). Further, also for $x=2 \lambda$, we have

$$
\cos \theta_{k}=\frac{\varepsilon_{1}-\varepsilon_{2}}{|\underline{u}|}
$$

from (A.32), from which

$$
\begin{aligned}
\sin \theta_{k} & =\frac{\sqrt{\underline{u}^{2}-\left(\varepsilon_{1}-\varepsilon_{2}\right)^{2}}}{|\underline{u}|} \\
& =\frac{\sqrt{2 \lambda}}{|\underline{u}|} .
\end{aligned}
$$

Substituting these values for $\omega, \cos \theta_{k}$, and $\sin \theta_{k}$ in the expressions (24) for $a^{\prime}$ and $b$ we have, for $x=2 \lambda$,

$$
a^{\prime}=\frac{k}{\underline{u}^{2}}\left[\varepsilon_{1} \underline{u}^{2}-p_{1}\left(\varepsilon_{1}-\varepsilon_{2}\right)\left(p_{1}-p_{2} \cos \theta\right)\right]
$$

and

$$
b=-\frac{\kappa p_{1} p_{2} \sin \theta \sqrt{2 \lambda}}{\underline{u}^{2}} .
$$

In writing (A.61) we have used the expression for $\cos \theta_{s}$ given in (24), and in writing (A.62) we have used the expression for $\sin \theta_{p}$ given in (A.6). Recalling that $\underline{u}^{2}=p_{1}{ }^{2}-2 p_{1} p_{2} \cos \theta+p_{2}{ }^{2}$, a little algebra then gives 


$$
\begin{aligned}
a^{\prime} & =\frac{\kappa}{\underline{u}^{2}}\left(\varepsilon_{1}+\varepsilon_{2}\right)\left(\varepsilon_{1} \varepsilon_{2}-p_{1} p_{2} \cos \theta-m^{2}\right) \\
& =\frac{\kappa \lambda\left(\varepsilon_{1}+\varepsilon_{2}\right)}{\underline{u}^{2}} .
\end{aligned}
$$

Substituting (A.63) and (A.62) in (A.25) we then have

$$
D_{2}(2 \lambda)=\frac{4 k^{2} \lambda}{\underline{u}^{2}}\left[\lambda\left(\varepsilon_{1}+\varepsilon_{2}\right)^{2}-2 p_{1}{ }^{2} p_{2}{ }^{2} \sin ^{2} \theta\right] .
$$

Again recalling that $\lambda=\varepsilon_{1} \varepsilon_{2}-p_{1} p_{2} \cos \theta-m^{2}$, a little algebra gives

$$
\begin{aligned}
\lambda\left(\varepsilon_{1}+\varepsilon_{2}\right)^{2}-2 p_{1}{ }^{2} p_{2}{ }^{2} \sin ^{2} \theta & =\left(\varepsilon_{1} \varepsilon_{2}-p_{1} p_{2}+m^{2}\right)\left(p_{1}{ }^{2}-2 p_{1} p_{2} \cos \theta+p_{2}{ }^{2}\right) \\
& =\left(\lambda+2 m^{2}\right) \underline{u}^{2},
\end{aligned}
$$

so that

$$
D_{2}(2 \lambda)=4 k^{2} \lambda\left(\lambda+2 m^{2}\right)
$$

and

$$
B=D_{1}^{\frac{1}{2}}(2 \lambda)=D_{2}^{\frac{3}{2}}(2 \lambda)=2 k \sqrt{\lambda\left(\lambda+2 m^{2}\right)} .
$$

54 


\section{REFERENCES}

[1] Y. S. Tsai, in Proceedings of the International Conference on Nuclear Structure, 1963 edited by Hofstadter and Schiff (Stanford Univ. Press, Stanford, California, 1964), p. 221.

[2] L. W. Mo and Y. S. Tsai, Rev. Mod. Phys. 41, 205 (1969).

[3] Y. S. Tsai, SLAC-PUB 848, January 1971.

[4] G. Miller, et al. Phys. Rev. D5, 528 (1972).

[5] L. C. Maximon and D. B. Isabelle, Phys. Rev $\underline{133}, B 1344$ (1964).

[6] J. D. Bjorken and S. D. Drell, Relativistic Quantum Mechanics (McGraw Hill Book Co., New York, 1964), see Chap. 7.

[7] Stein et a1., Phys. Rev. D12, 1884 (1975). In eq (A24) of this reference, the authors have corrected two misprints that appear in eq (A.24) of SLAC-PUB 848, reference 3 above.

[8] H. Nguyen-Ngoc and J. P. Perez-y-Jorba, Phys. Rev. 136, B1036 (1964).

[9] W. Gröbner and N. Hofreiter, Integraltafel, Part I (Springer, Vienna, 1961). 
The form factors used in these timing runs were derived from a DWBA calculation for ${ }^{12} \mathrm{C}$ at $500 \mathrm{MeV}$ (see subroutine PWAFFI in the computer program listing, pp. 98-100).

PWA denotes the piece-wise analytic evaluation of the integral as presented in this report.

All times listed are in seconds. Numerical integrals were calculated with an accuracy of $0.0001 \%$.

$p_{1}=250 \mathrm{MeV}$

1 deg.

$\mathrm{P}_{2}(\mathrm{MeV})$

10

125

248

$5 \mathrm{deg}$

$\begin{array}{ll}\mathrm{p}_{2}(\mathrm{MeV}) & \text { Simpson } \\ 10 & 11.66 \\ 125 & 4.37 \\ 240 & 1.86\end{array}$

45 degi

$\mathrm{p}_{2}$ (MeV)

10

125

240

90 deg.

$\mathrm{P}_{2}(\mathrm{MeV})$

10

125

240

135 deg.

$\mathrm{p}_{2}(\mathrm{MeV})$

10

125

240

175 deg.

$\mathrm{p}_{2}(\mathrm{MeV})$

10

125

Simpson
21.87
62.03
3.15

\section{Simpson}

2.46

2.33

2.21

Simpson

3.81

2.61

2.46
Simpson
5.35
3.15
3.38

simpson

11.71

4.47

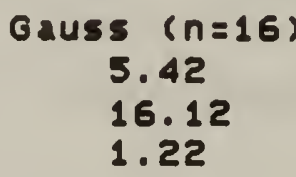

Gauss $(n=16)$

5.42

16.12

1.22
Gauss $(n=16)$ Kronrod $(n=61)$
2.86
1.30
.59
.96
.96
.61

1.57

2.36

.85

.43

.22

.02

$\begin{array}{ccc}\text { Gauss }(n=16) & \text { Kronrod }(n=61) & \text { PWA } \\ .84 & .87 & .43 \\ 1.08 & .78 & .23 \\ .87 & .80 & .02\end{array}$

Gauss $(n=16)$ Kronrod $(n=61)$

PWA

1.24

.93

.44

1.17

1.23

.84

.23

.89

.82

Gauss $(n=16)$ Kronrod $(n=61)$

PWA

1.78

1.37

1.17

.92

.43

$1 \cdot 31$

.97

.01

Gauss $(n=16)$ Kronrod $(n=61)$

PWA

2.43

1.06

1.18

1.73

.23 
All times are in seconds. Numerical integrals were calculated with an accuracy of $8.0001 \%$.

$p_{1}=500 \mathrm{MeV}$

1 deg.

$\mathrm{p}_{2}(\mathrm{MeV})$

10

250

490

5 deg.

$\mathrm{p}_{2}(\mathrm{MeV})$

10

250

490

45 deg.

$\mathrm{p}_{2}(\mathrm{MeV})$

10

250

490

90 deg.

$\mathrm{p}_{2}(\mathrm{MeV})$

16

250

135 deg.

$\mathrm{P}_{2}(\mathrm{MeV})$

10

250

175 deg.

$\mathrm{p}_{2}(\mathrm{MeV})$

10

250

Simpson
48.45
70.24
3.66

Simpson

33.19

6.57

3.35

Simpson
3.26
3.76
5.01

Simpson

6.48

4.31

Simpson

20.92

6.40

Simpson

23.49

8. 70

$\begin{array}{cc}\text { Gauss }(n=16) & \text { Kronrod }(n=61) \\ 8.53 & 2.39 \\ 16.29 & 2.90 \\ 1.20 & 1.01\end{array}$

PWA

.99

.44

.03

$\begin{array}{ccc}\text { Gauss }(n=16) & \text { Kronrod }(n=61) & \text { PWA } \\ 3.06 & 2.21 & .99 \\ 2.16 & 1.32 & .44 \\ 1.24 & 1.84 & .03\end{array}$

Gauss $(n=16)$ Kronrod $(n=61) \quad$ PWA

1.31

.75

1.33

2.32

1.96

.98

.46

.02

$\begin{array}{ccc}\text { Gauss }(n=16) & \text { Kronrod }(n=61) & \text { PWA } \\ 2.33 & 1.29 & .99 \\ 1.75 & 1.55 & .48\end{array}$

Gauss $(n=16)$ Kronrod $(n=61)$

PWA

3.61

1.97

.99

2.58

1.44

.53

Gauss $(n=16)$ Kronrod $(n=61)$

PWA

5.90

3.46

1.86

1.89

1.00

.56 
All times listed are in seconds. Numerical integrals were calculated with an accuracy of $0.0081 x$.

$p_{1}=750 \mathrm{MeV}$

1 deg.

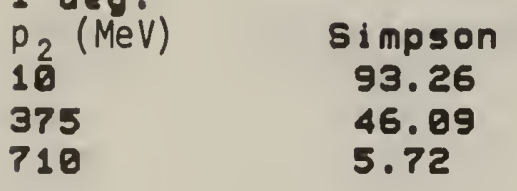
Gauss $(n=16)$ Kronrod $(n=61)$
2.52
18.85
18.82
2.96
1.86
1.23

PWA

1.63

.78

.88

5 deg.

$\begin{array}{ll}\mathrm{p}_{2}(\mathrm{MeV}) & \text { Simpson } \\ 16 & 59.96 \\ 375 & 7.26 \\ 710 & 4.49\end{array}$

Gauss $(n=16)$

Kronrod $(n=61)$

PWA

15. 38

3.94

2.33

1.36

1.89

1.27

1.63

.78

.88

45 deg.

$\mathrm{P}_{2}(\mathrm{MeV})$

Simpson

5.66

5.00

5.68

Gauss $(n=16)$

Kronrod $(n=61)$

PWA

375

2. 33

2.89

1.82

2.57

1.75

1.30

1.62

.75

.87

$\begin{array}{ll}90 \text { deg. } & \\ \mathrm{p}_{2} \text { (MeV) } & \text { Simpson } \\ 18 & 11.47 \\ 375 & 6.68\end{array}$

$135 \mathrm{deg}$

$\begin{array}{ll}\mathrm{p}_{2}(\mathrm{MeV}) & \text { simpson } \\ 16 & 26.18 \\ 375 & 9.84\end{array}$

Gauss $(n=16)$

Kronrod $(n=61)$

PWA

$$
4.48
$$

3.44

1.92

1.62

1.21
Gauss $(n=16)$
7.16
2.36
2.39
2.14

Kronrod $(n=61)$

\section{PWA}

1.62

.98

175 deg.
$\mathrm{p}_{2}(\mathrm{MeV})$
18
375

Simpson
57.47
18.22

Gauss $(n=16)$

15.11

3.56

Kronrod $(n=61)$

PHA

1.62

.88 


\section{COMPUTER PROGRAM LISTING}

In the pages which follow we give the listing of the computer program. However, the subroutines PROMPT, TOD, and CLK (which are called in the main program MSPLTIM, as well as in the timing subroutines STARTT and STOPT) are system information routines which are unique to the Perkin-Elmer system on which the program was run. They are not, therefore, inciuded in the program listing. 


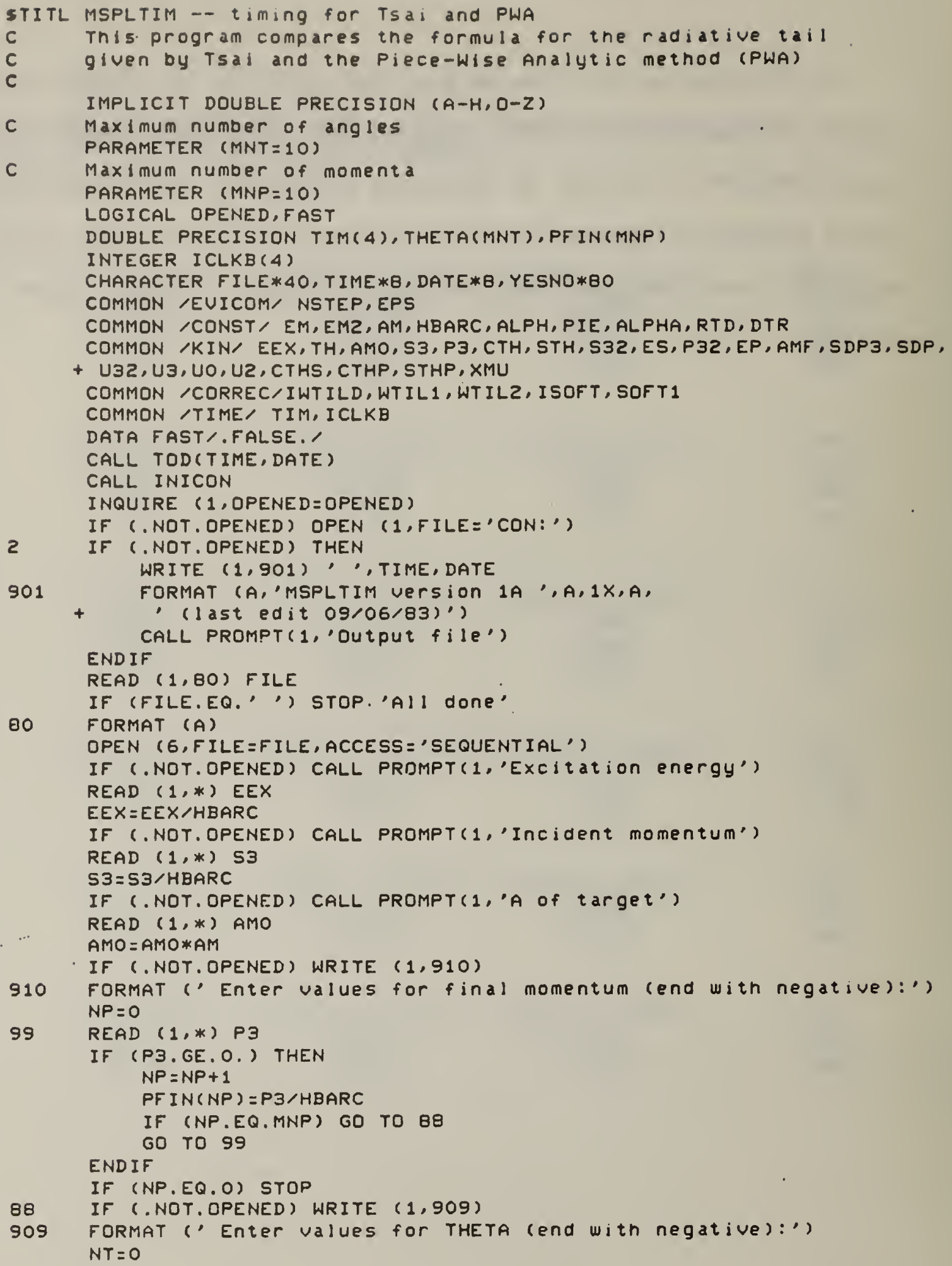




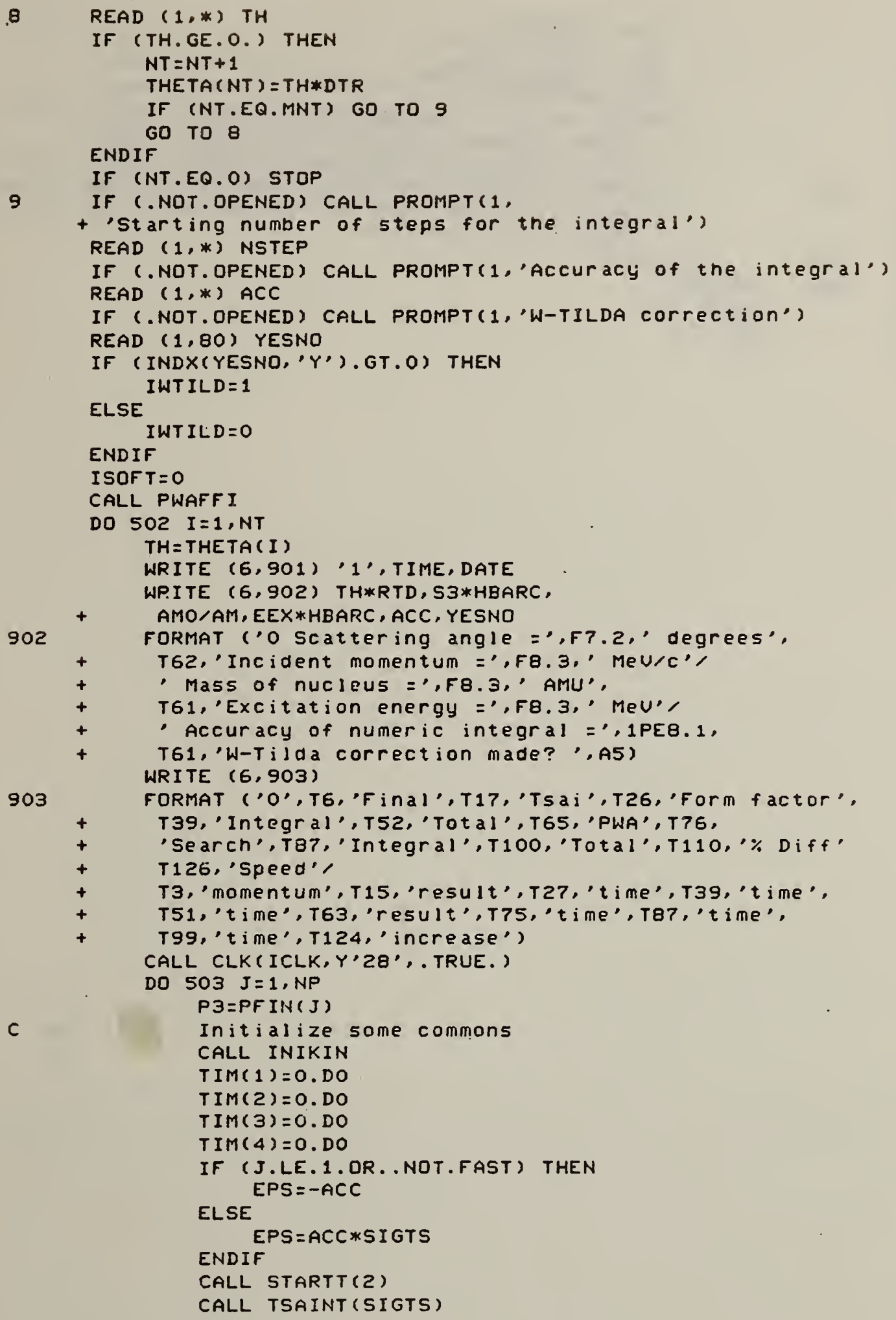




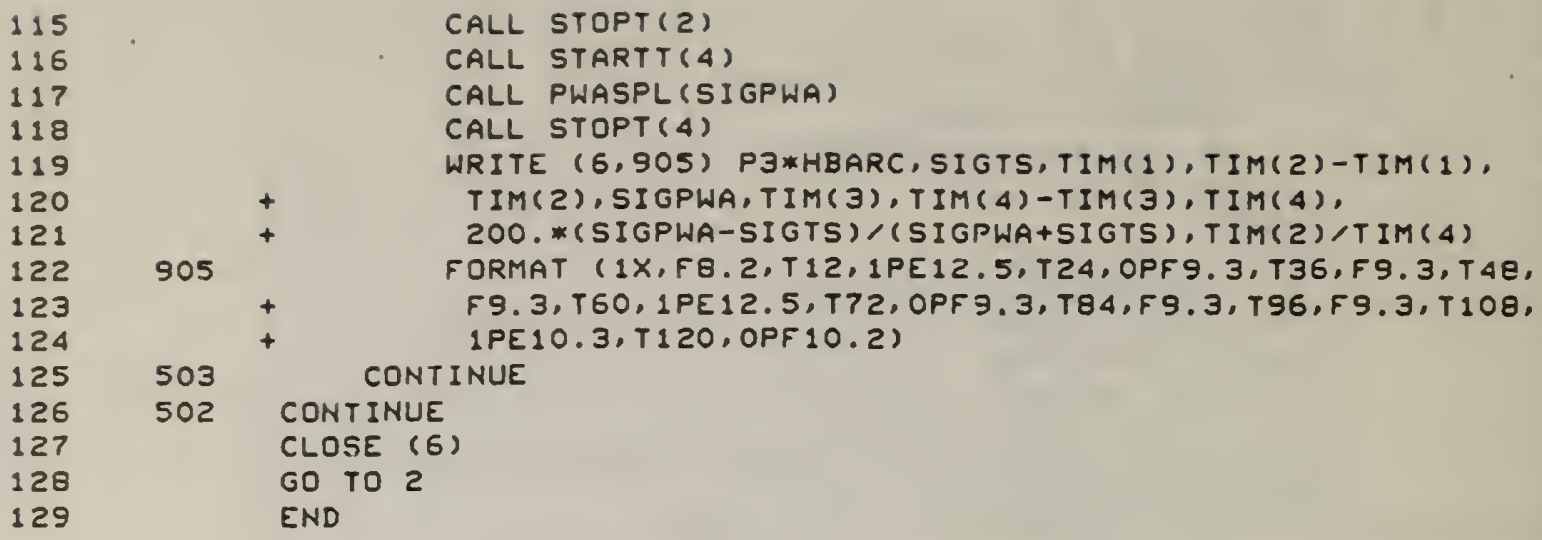

CALL STOPT( 2$)$

CALL STARTT (4)

CALL PWASPL(SIGPWA)

CALL STOPT (4)

WRITE (6,905) P3*HBARC, SIGTS, TIM(1), TIM(2)-TIM(1), $\operatorname{TIM}(2), 5 I G P W A, T I M(3), \operatorname{TIM}(4)-\operatorname{TIM}(3), \operatorname{TIM}(4)$, 200.*(SIGPWA-SIGTS) / (SIGPWA+SIGTS), TIM(2)/TIM( 4 ) 


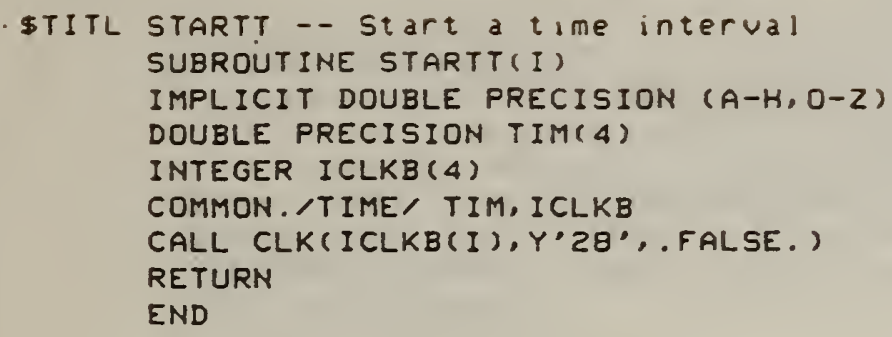




\begin{tabular}{|c|c|c|}
\hline 1 & STITL & STOPT - - stop a time interval \\
\hline 2 & & SUBROUTINE STOPT(I) \\
\hline $\begin{array}{l}3 \\
4\end{array}$ & C & $\begin{array}{l}\text { Note that the constant is for optimized Fortran. } \\
\text { IMPLICIT DOUBLE PRECISION }(A-H, O-Z)\end{array}$ \\
\hline 5 & & DOUBLE PRECISION TIM(4) \\
\hline 6 & & INTEGER ICLKB (4) \\
\hline 7 & & COMMON /TIME/ TIM, ICLKB \\
\hline 8 & & CALL CLK(ICLKE, Y' $28^{\prime}$, . FALSE. ) \\
\hline 9 & & $\operatorname{TIM}(I)=\operatorname{TIM}(I)+(\operatorname{ICLKE}-\operatorname{ICLKB}(I)) / 1000 .-.0009374$ \\
\hline 10 & & RETURN \\
\hline 11 & & END \\
\hline
\end{tabular}




\begin{tabular}{|c|c|c|}
\hline $\begin{array}{l}1 \\
2\end{array}$ & \$TITL & $\begin{array}{l}\text { INICON -- Initialize constants } \\
\text { SUBROUTINE INICON }\end{array}$ \\
\hline 3 & C & subroutine to initialize the common CONST. All energies in \\
\hline 4 & C & units of $f m-1$. Constants from RMP 52. No. 2 (Aprii 1980) as \\
\hline 5 & C & reprinted by the CERN particle properties data booklet. \\
\hline 6 & & IMPLICIT DOUBLE PRECISION (A-H,O-Z) \\
\hline 7 & & COMMON /CONST, EM, EMZ, AM, HBARC, ALPH, PIE, ALPHA, RTD, DTR \\
\hline 8 & & HBARC $=197.32858 D 0$ \\
\hline 9 & & $A L P H=137.03604 D 0$ \\
\hline 10 & & $A M=931.5016 D 0 / H B A R C$ \\
\hline 11 & & $E M=.5110034 \mathrm{DO} / \mathrm{HBARC}$ \\
\hline 12 & & $E M 2=E M * * 2$ \\
\hline 13 & & $P I E=3.1415926535897 \mathrm{DO}$ \\
\hline 14 & & $A L P H A=1 . O D O / A L P H$ \\
\hline 15 & & $D T R=P I E / 180 . D O$ \\
\hline 16 & & RTD $=180 . D O / P I E$ \\
\hline 17 & & RETURN \\
\hline $1 \varepsilon$ & & END \\
\hline
\end{tabular}




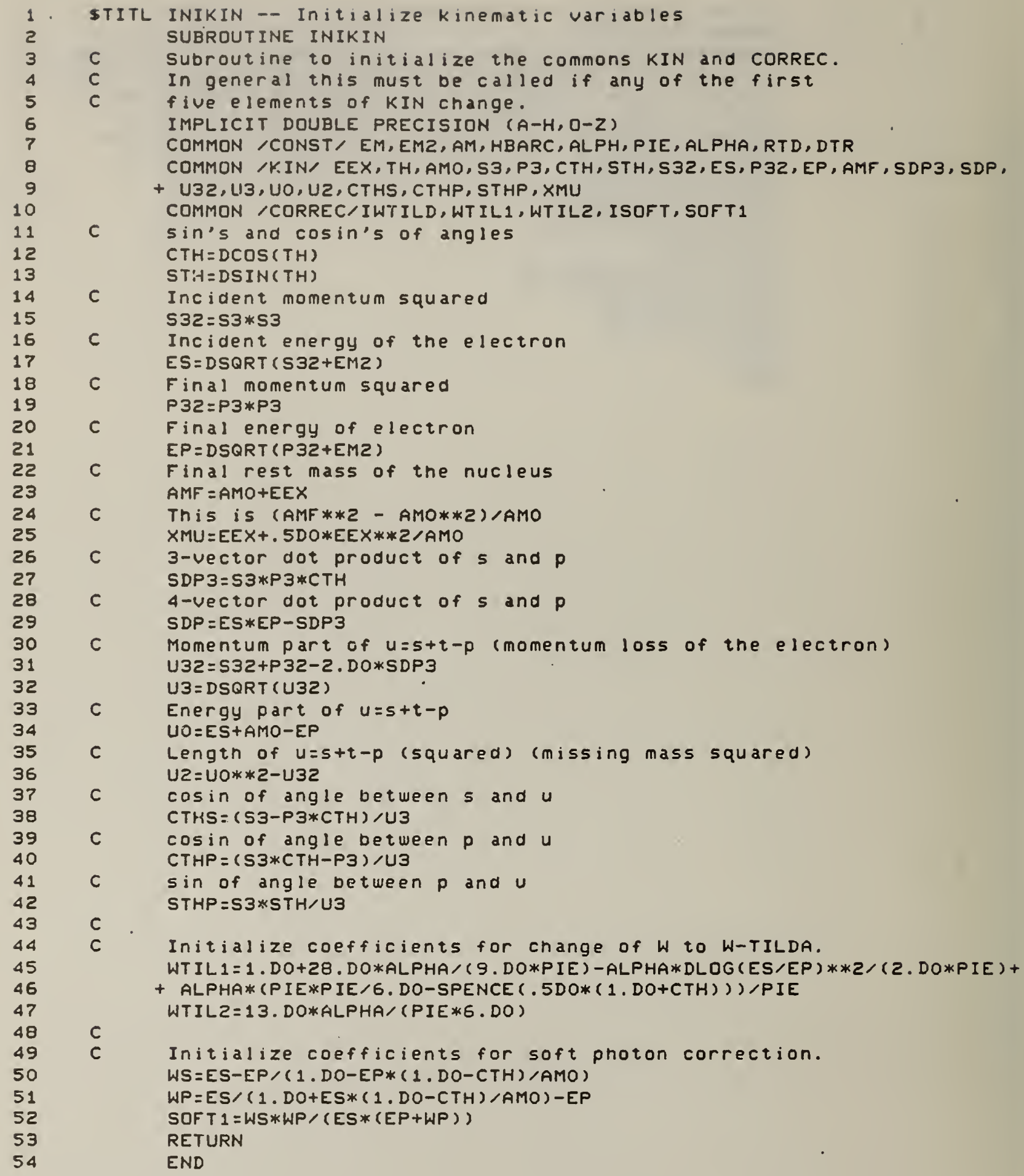


\$TITL TSAINT.-- Evaluate TSai by doing numerical integral SUBROUTINE TSAINT (UAL)

C The formula given by Tsai in 51 ac-plib 848 (as we 11 as in

C Mo and TSai RMP 41, 205 and stein et. a1. Phys Reu D 12, 1884)

C is evaluated by doing the integral numerically. This

C is done in up to ? separate intervals. Cal.1 INIKIN before

c calling this routine.

IMPLICIT DOUBLE PRECISION $(A-H, O-Z)$

EXTERNAL TSAI

LOGICAL DEBUG

INTEGER NS(?)

DOUBLE PRECISION EDGE (8)

COMMON /CONST/ EM, EM2, AM, HBARC, ALPH, PIE, ALPHA, RTD, DTR

COMMON /KIN/ EEX,TH,AMO,S3,P3,CTH,STH,S32, ES,P32, EP, AMF, SDP3, SDP,

+ U32, U3, UO, U2, CTHS, CTHP, STHP, XMU

DATA DEBUG/.FALSE. $/$

DATA EDGE (1)/-1.DO/.EDGE (8)/1.DO/

UAL $=0 . D O$

THLIM $=A M O *(E S-E P) /(2 . D O * E S * E P)$

IF (THLIM.LE. 1.DO) THEN

THLIM = 2. DO*DASIN (DSQRT (THLIM))

ENDIF

IF (TH.GT.THLIM) RETURN

THS = DATANZ (DSQRT (1.DO-CTHS**2), CTHS)

DTHS = DSQRT (EM/ES) *2.ODO

THP = DATANZ (STHP, CTHP)

DTHP $=D S Q R T$ (EM/EP) *2. ODO

$E D G E(2)=D C O S(D M I N 1(P I E, T H P+D T H P))$

EDGE (3) =CTHP

$\operatorname{EDGE}(4)=\operatorname{DCOS}(\operatorname{DMAX1}(0 . D 0$, THP $-D T H P))$

EDGE (5) $=D C O S(D M I N 1(P I E, T H S+D T H S)$ )

EDGE ( 6$)=$ CTHS

EDGE $(7)=D C O S(D M A X 1(0 . D O, T H S-D T H S))$

IF (EDGE (4).GT.EDGE (5)) THEN

$\operatorname{EDGE}(4)=(\operatorname{EDGE}(3)+\operatorname{EDGE}(6)) / 2 . D O$

ENDIF $\operatorname{EDGE}(5)=\operatorname{EDGE}(4)$

DO $504 I=1,7$

$N S(I)=0$

IF (EDGE (I).LT.EDGE $(I+1)$ ) THEN

DSIG = EUALI (EDGE (I), EDGE (I+1), TSAI, NS(I))

IF (DEBUG) WRITE $(1, *)$ CHAR(ICHAR('A') +I-1),

$+$

EDGE (I), EDGE $(I+1)$, DSIG

ENDIF

504 CONTINUE

UAL $=U A L * A L P H A * * 3 * P 32 * A M O /$ (PIE*EP*S3)

IF (DEBUG) WRITE (1,31) NS, UAL

31

FORMAT $(1 X, 7$ I5, E10.3)

RETURN

END 


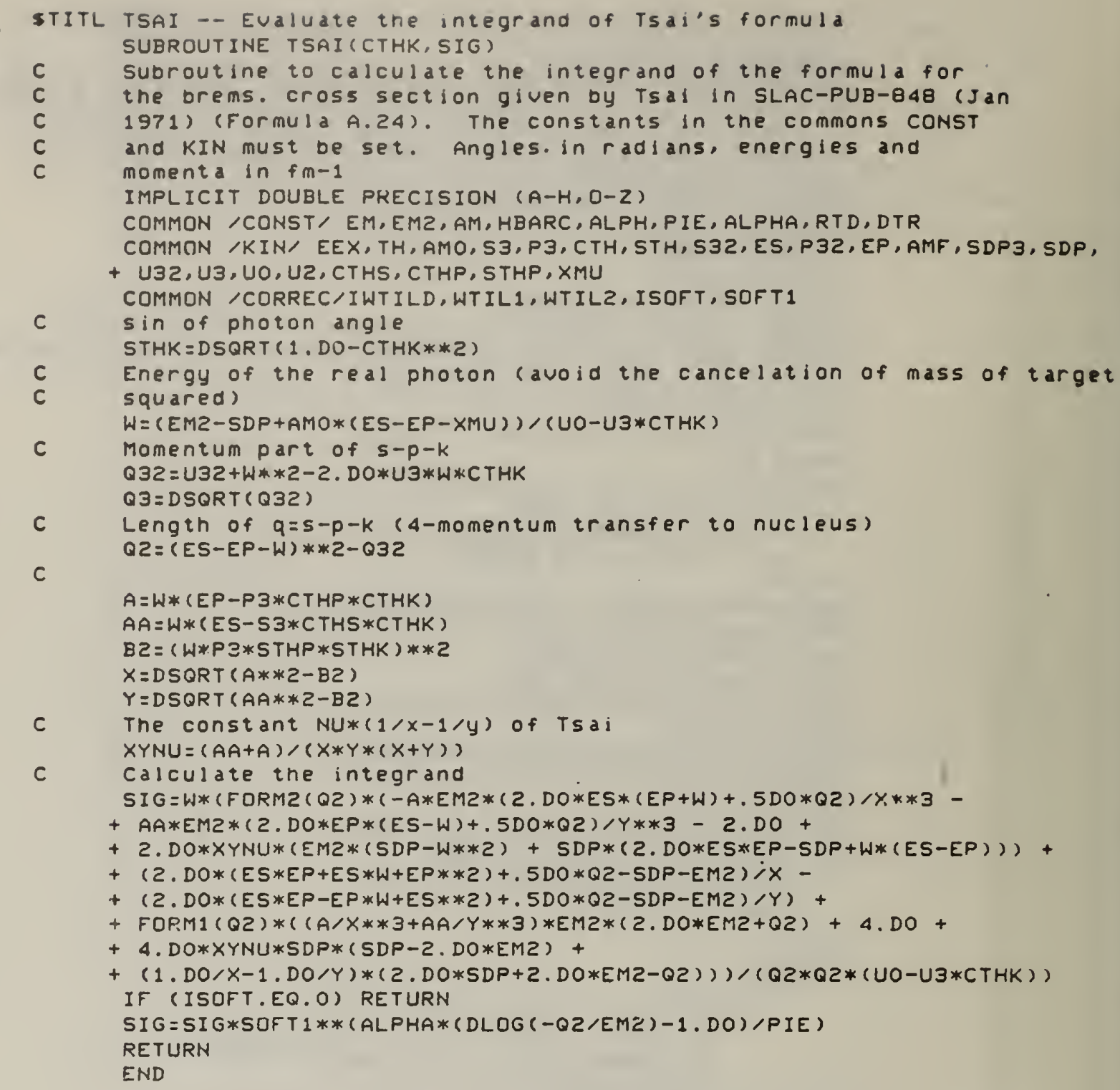




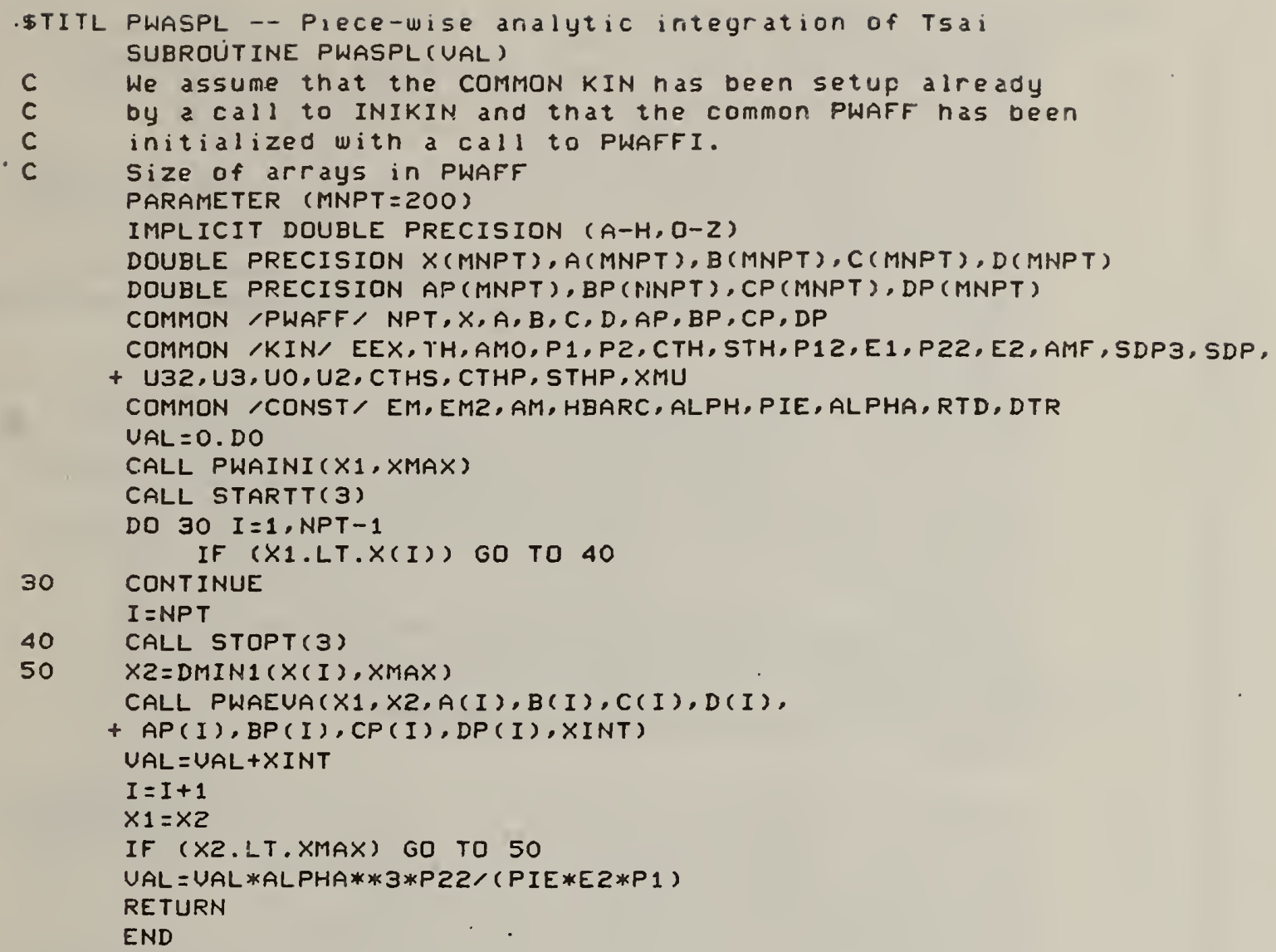


\$TITL PWAINI, PWAEUA -- AnaJytiC TSaI, CUbic form factor SUBROUTINE PWAINI ( $X M I N, X M A X)$

C PWAINI should be called once, after the common KIN has

C been initialized in order to calculate constants which

C do not change when only THETA-K (or q-MU) changes.

IMPLICIT DOUBLE PRECISION $(A-Z)$

COMMON /PWACOM/K1M2,K1M1,K10,K1P1,K1P2,K1P3,K2M2,K2M1,K20,

$+K 2 P 1, K 2 P 2, K 2 P 3, J 1 M 2, J 1 M 1, J 10, J 1 P 1, J 1 P 2, J 2 M 2, J 2 M 1, J 20, J 2 P 1$,

+ JZPZ,L,LAMBDA

COMMON /CONST, EM, EMZ, AM, HBARC, ALPH,PIE, ALPHA, RTD, DTR

COMMON /KIN/ EEX, TH,AMO,P1,P2,CTH,STH,P12,E1,P22,EZ,AMF, SDP3, SDP,

+ U32,U3, UO,U2, CTHS, CTHP, STHP, XMU

LAMBDA $=5 D P-E M 2$

KAPPA $=E 1-E Z-X M U-L A M B D A / A M O$

CALL PWACOE (LAMBDA, KAPPA, P1, E1,P2,E2, 1.D0, A1, B1, C1, ETA1,

+ XMIN, BETO, BET1, BET2, TAU1, RHO1, BETOP, BET1P, BET2P)

CALL PWACOE (LAMBDA, KAPPA, P2, E2, P1, E1, - 1. DO, A2, B2, C2, ETA2,

+ XMAX, DELO, DEL 1, DEL2, TAU2, RHO2, DELOP, DEL1P, DEL2P) GAMMA $=($ (LAMBDA+EMZ)*(2.DO*E $1 * E 2-L A M B D A)+L A M B D A * K A P P A * * 2+$

$+(L A M B D A+E M Z) *(L A M B D A / A M O+X M U) * K A P P A) / L A M B D A * * 2$

$E T A=1 . D O$

TAUP $=-2 . D O *(L A M B D A * * 2-2 . D O * E M 2 *(L A M B D A+E M 2)) / L A M B D A$

GAMMAP $=2 . D O *(L A M B D A * * 2-E M 2 * * 2) / L A M B D A * * 2$

$E T A P=-2 . D O$

RHOP $=(L A M B D A * * 2-2 . D O * E M 2 * * 2) / L A M B D A * * 2$

RETURN

C

PWAEUA calculates the integral of Tsai from XI to XF assuming

that the longitudinal form factor (W2) is a cubic defined

by $A, B, C$, and $D$ and that the transverse form factor (W1)

is a cubic given by $A P, B P, C P$, and $D P$.

ENTRY PWAEUA ( $X I, X F, A, B, C, D, A P, B P, C P, D P, U A L)$

CALL PWAKINCXI, XF, LAMBDA, KAPPA, P1, E1,P2, E2, ETA1, A1, B1, C1,

$\left.+D_{1} I, D 1 F, J 10, J 1 M 1, J 1 P 1, J 1 M 2, J 1 P 2, K 10, K 1 M 1, K 1 P 1, K 1 M 2, K 1 P 2, K 1 P 3\right)$

CALL PWRKINGXI, XF, LAMBDA, KAPPA, P2,E2,P1, E1, ETA2, A2, B2, C2,

$+D 2 I, D 2 F, J 20, J 2 M 1, J 2 P 1, J 2 M 2, J 2 P 2, K 20, K 2 M 1, K 2 P 1, K 2 M 2, K 2 P 2, K 2 P 3$ ) $B E T A=2 . D O * K A P P A * D S Q R T$ (LAMBDA*(LAMBDA+2.DO*EMZ))

ZETA1 $I=(2.0 D O * L A M B D A * A 1-B 1) * X I-2 . D O * L A M B D A * B 1+C 1$

$Z F T A 1 F=(2.0 D 0 * L A M B D A * A 1-B 1) * X F-2 . D O * L A M B D A * B 1+C 1$

ZETAZI $=(2.0 D O * L A M B D A * A Z-B Z) * X I-2 . D O * L A M B D A * B 2+C 2$

ZETAZF $=(2.0 D O * L A M B D A * A Z-B Z) * X F-2 . D O * L A M B D A * B 2+C 2$

$c$
$C$
$C$
$C$ This is the formula as originally written in Maximon's notes $L=-D L O G($ (ZETAZF+BETA*DZF) / (ZETA1F+BETA*D1F)*

$+(Z E T A 1 I+B E T A * D 1 I) /(Z E T A 2 I+B E T A * D 2 I)) / B E T A$

The following version of $L$ is rewritten to avoid cancelation $S 1 I=1 . D O$

EPS $1 I=0$. DO

IF (ZETAII.LT.O.DO) THEN

$S 1 I=-1 . D O$

ENDIF

EPSII $=2 . D O$

$S 2 I=1 . D O$

EPSZI $=0 . D O$

IF (ZETAZI.LT.O.DO) THEN

$S 2 I=-1$. DO

ENDIF

EPS2I $=2$. DO 


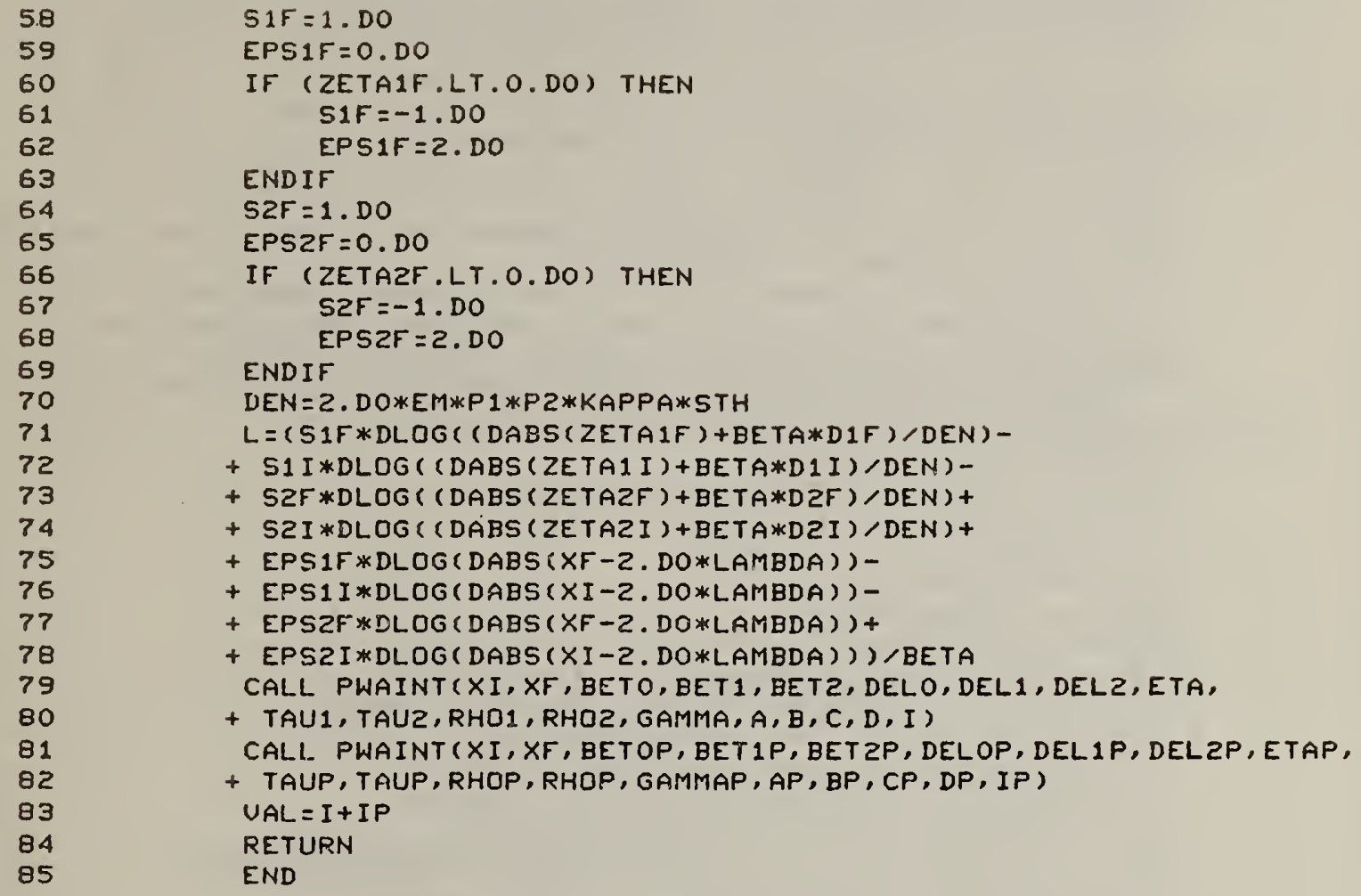




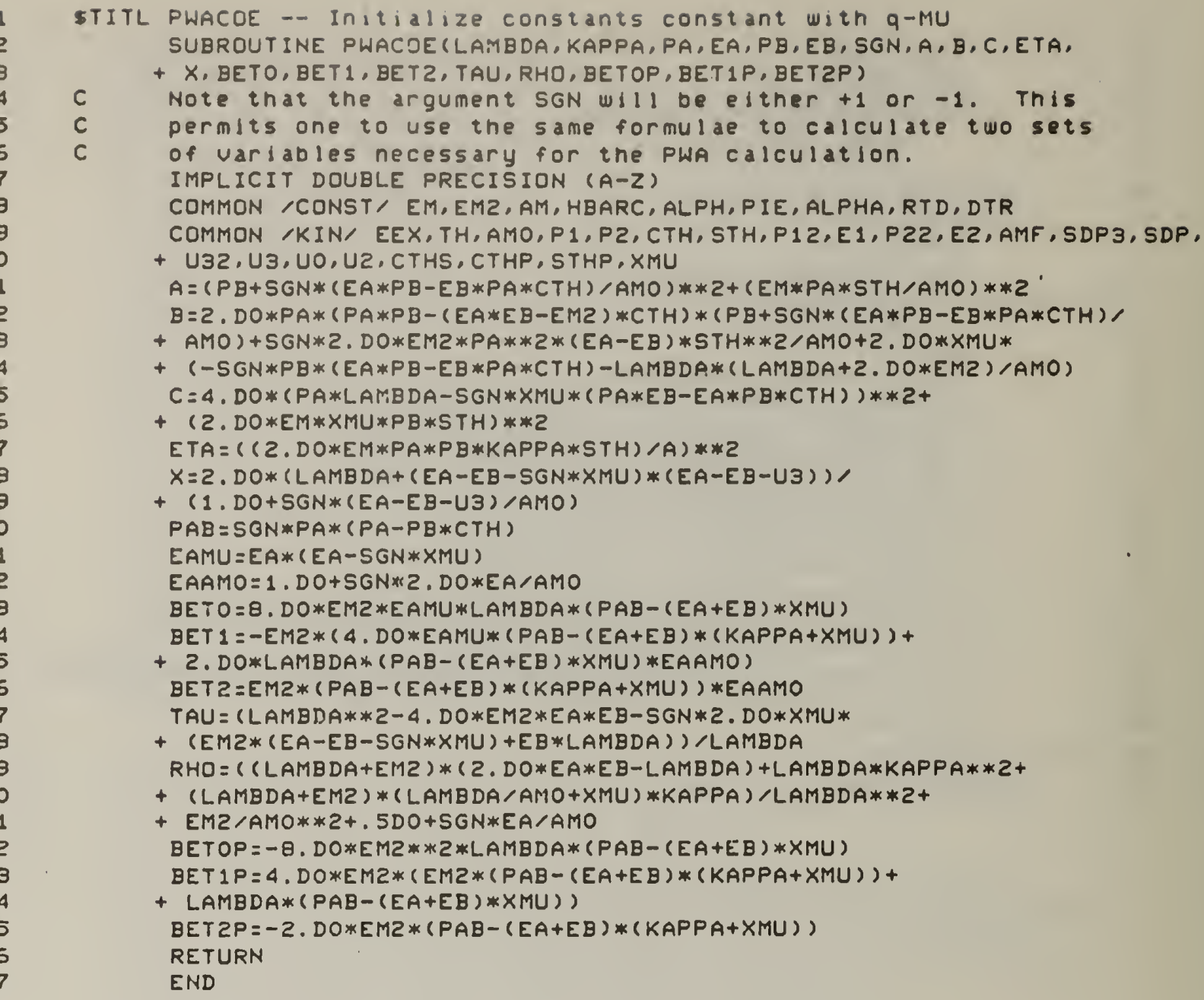


\$TITL PWAKIN - Evaluate constants which change with q-MU SUBRDUTINE PWAKINCXI, XF, LAMBDA, KAPPA, PA, EA, PB, EB, ETA, A, B, C,

$+D I, D F, J 0, J M 1, J P 1, J M 2, J P 2, K O, K M 1, K P 1, K M 2, K P 2, K P 3)$

IMPLICIT DOUBLE PRECISION $(A-Z)$

COMMON /CONST/ EM,EMZ, AM, HBARC, ALPH,PIE, ALPHA, RTD, DTR

COMMON /KIN/ EEX, TH, AMO,P1,P2, CTH, STH,P12, E1,P22, E2, AMF, SDP3, SDP,

$+\mathrm{U} 32, \mathrm{U}, \mathrm{UO}, \mathrm{U2}, \mathrm{CTHS}, \mathrm{CTHP}, \mathrm{STHP}, \mathrm{XMU}$

$C H I=B / A$

$D I=D S Q R T(A *(X I-C H I) * * 2+E T A))$

$D F=D S Q R T(A *(X F-C H I) * * 2+E T A))$

SQTA $=$ DSQRT (A)

SQTC $=D S Q R T(C)$

$C$ The following is the formula as originally uritten

C JO=DLOG ( $A * X F-B+D F * S Q T A) /(A * X I-B+D I * S Q T A)) / S Q T A$

C This is the version as corrected to reduce "cancelation"

$S I=1.00$

If $(A * X I-B \cdot L T \cdot O \cdot D O) \quad S I=-1 . D O$

$S F=1 . D O$

IF $(A * X F-B . L T . O . D O) \quad S F=-1 . D O$

$D E N=2 . D O * E M * P A * P B * K A P P A * S T H$

$\mathrm{JO}=(\mathrm{SF} * \mathrm{DLOG}(\mathrm{DABS}(\mathrm{A} * X F-\mathrm{B})+\mathrm{DF} * S Q T A) / D E N)-$

$+5 I * D L O G((D A B S(A * X I-B)+D I * S Q T A) / D E N)) / S Q T A$

$C$ The following is the formula as originally uritten

C JM1 $=-D L D G(X I / X F *(-B * X F+C+5 Q T C * D F)$,

C $\quad+(-8 * X I+C+5 Q T C * D I)) / 5 Q T C$

C This version is corrected to avoid cancelation

$S I=1.00$

IF (B*XI-C.LT.O.DO) $S I=-1 . D O$

$S F=1 . D O$

IF $(B * X F-C . L T .0 . D O) \quad S F=-1 . D O$

$J M 1=(S F * D L O G((D A B S(B * X F-C)+S Q T C * D F) /(X F * D E N))-$

$+S I * D L D G((D A B S(B * X I-C)+S Q T C * D I) /(X I * D E N))) / S Q T C$ $J P 1=(D F-D I) / A+C H I * J O$

$J M C=(-D F / X F+D I / X I+B * J M 1) / C$

$J P Z=(X F * D F-X I * D I+3 . D O * B * J P 1-C * J O) /(2 . D O * A)$

$K O=((A * X F-B) / D F-(A * X I-B) / D I) /(E T A * A * * 2)$

$K P 1=-((-B * X F+C) / D F-(-B * X I+C) / D I) /(E T A * A * 2)$

$K P Z=(J O+2 . D O * B * K P 1-C * K O) / A$

$K P 3=(X F * 2 / D F-X I * 2 / D I+3 . D O * B * K P 2-2 . D 0 * C * K P 1) / A$

$K M 1=(1 \cdot D O / D F-1 \cdot D O / D I+J M 1+B * K O) / C$

$K M 2=(-1 . D O /(X F * D F)+1 . D O /(X I * D I)+3 . D O * B * K M 1-2 . D O * A * K O) / C$ RETURN

END 


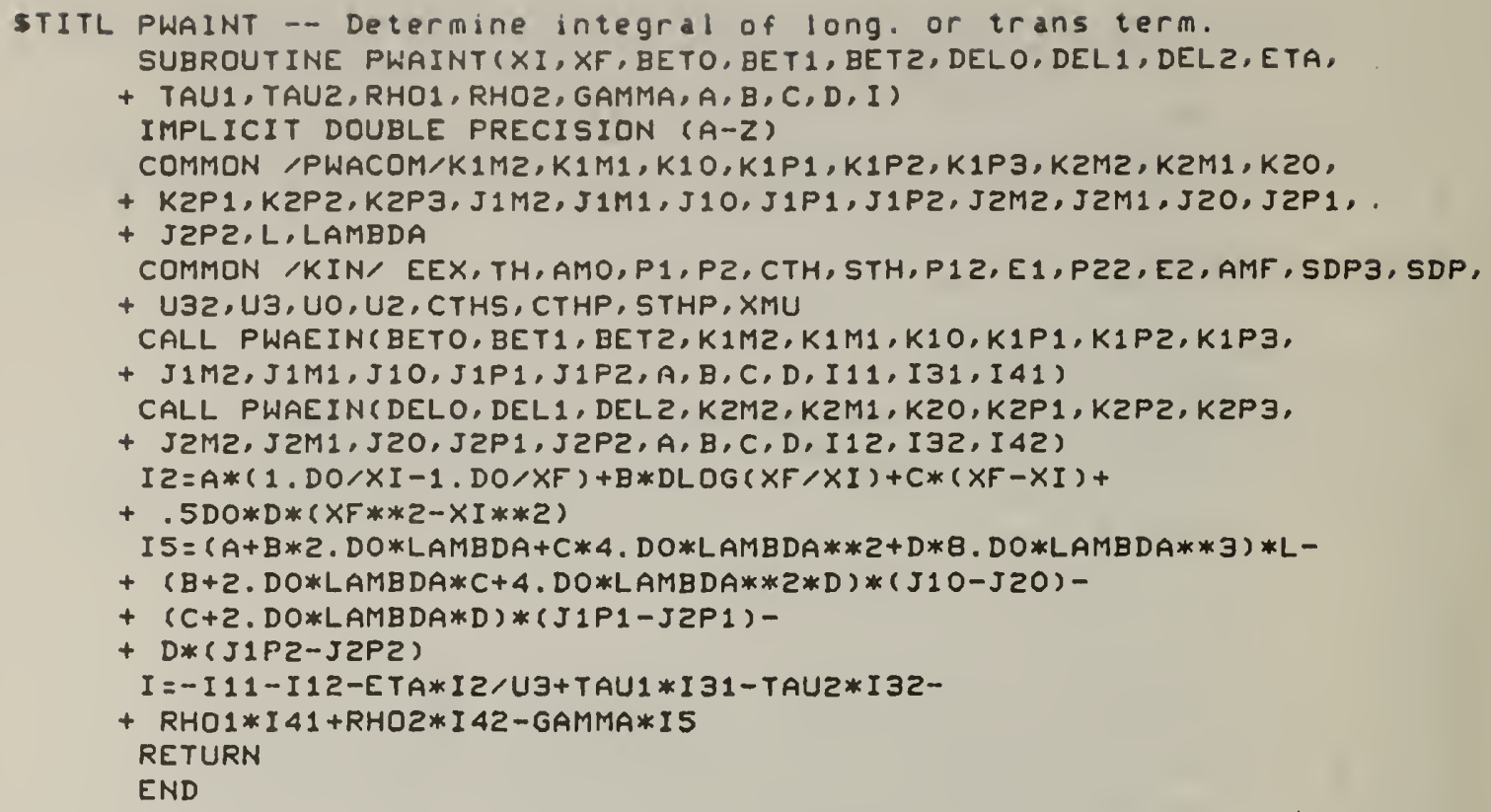

74 


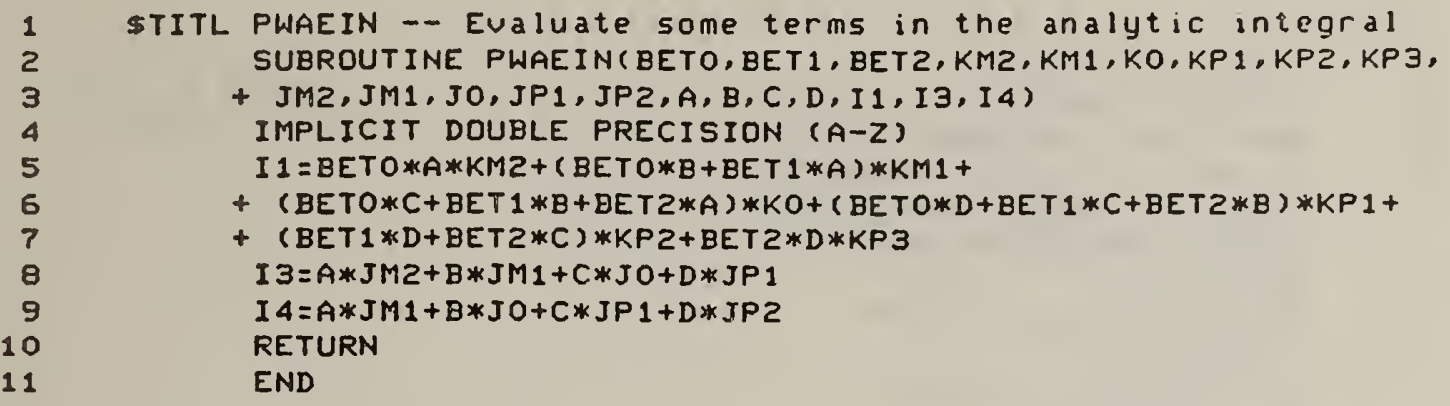




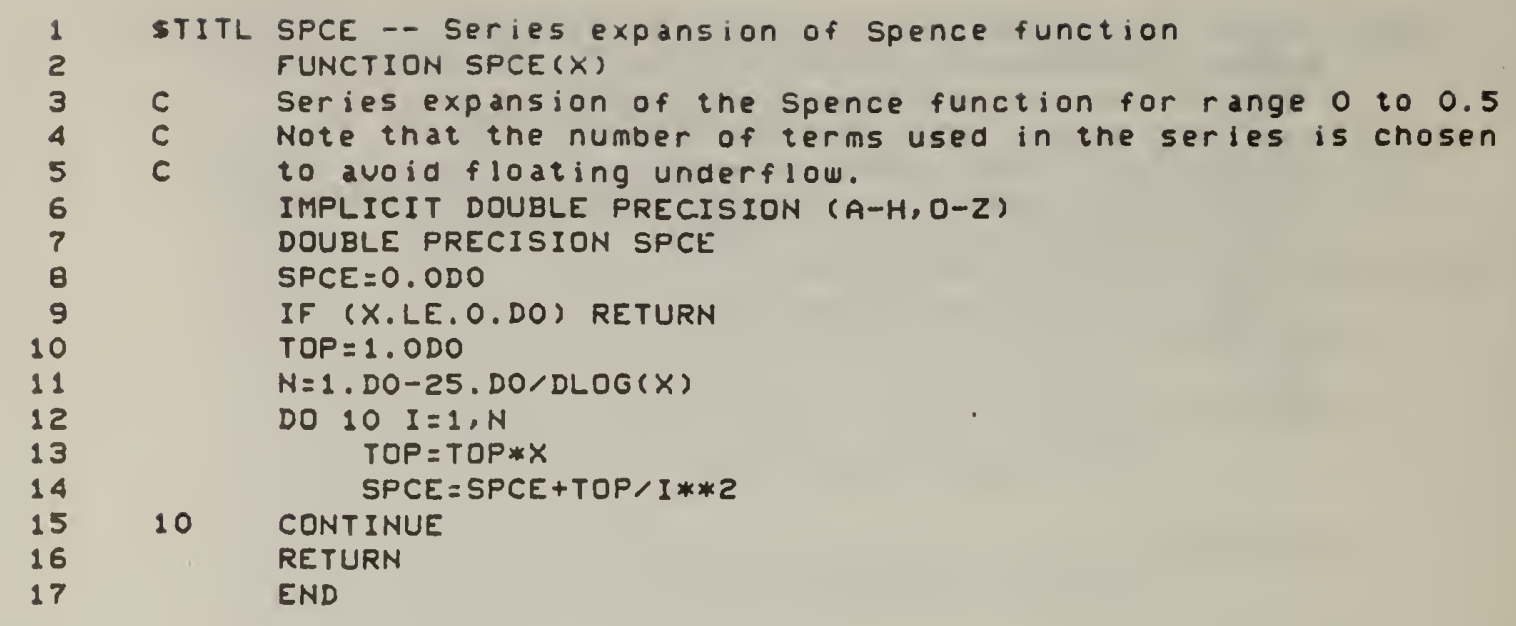




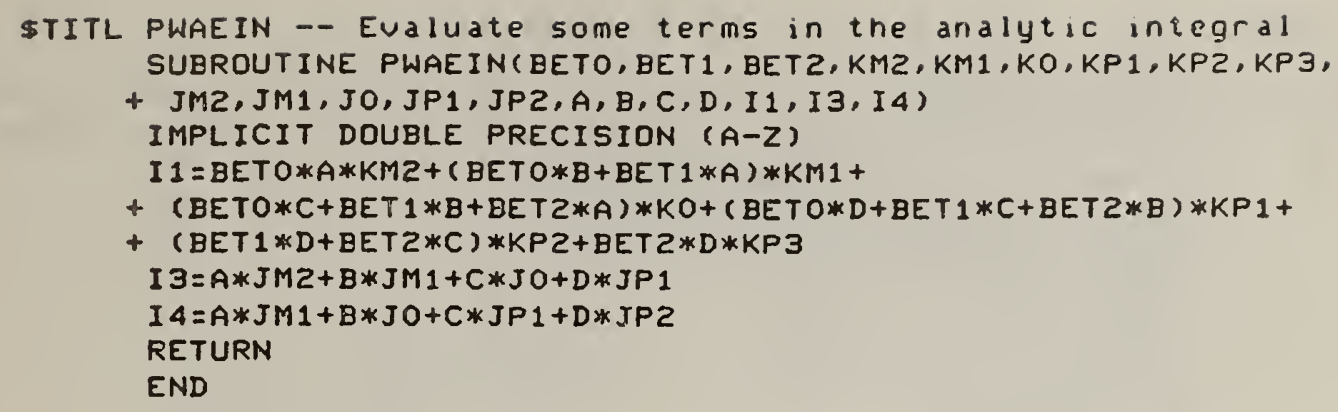




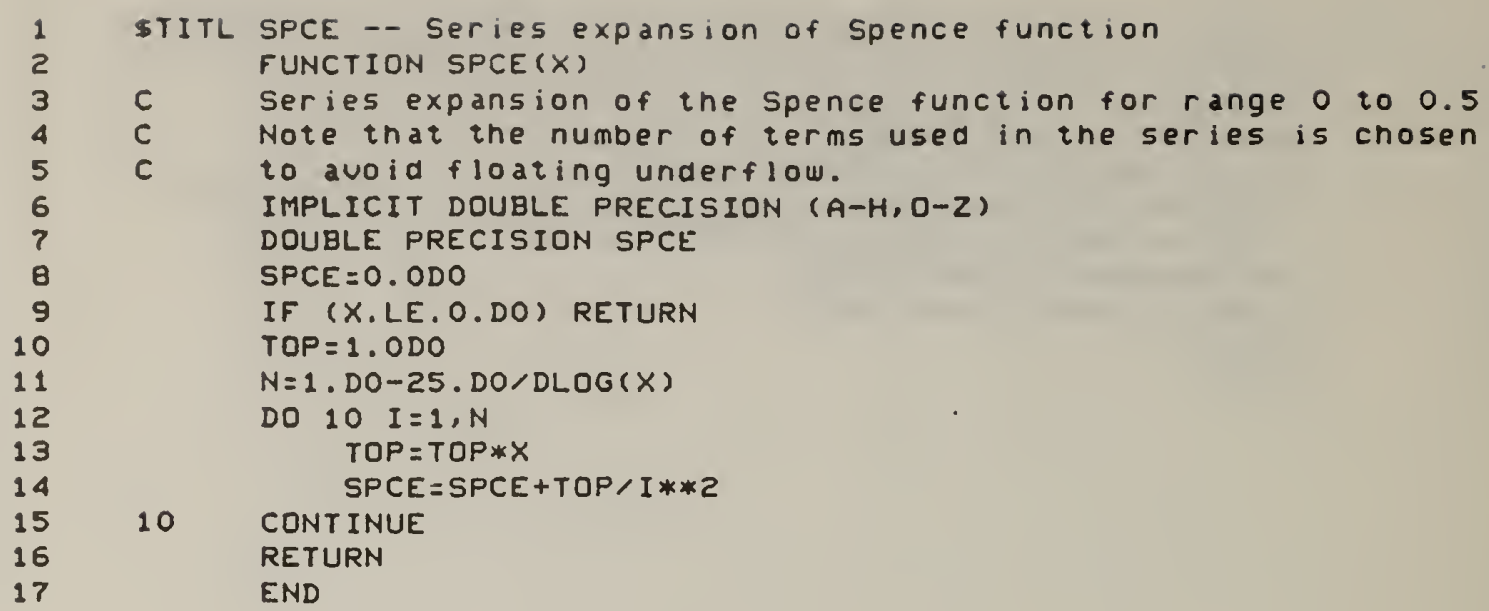




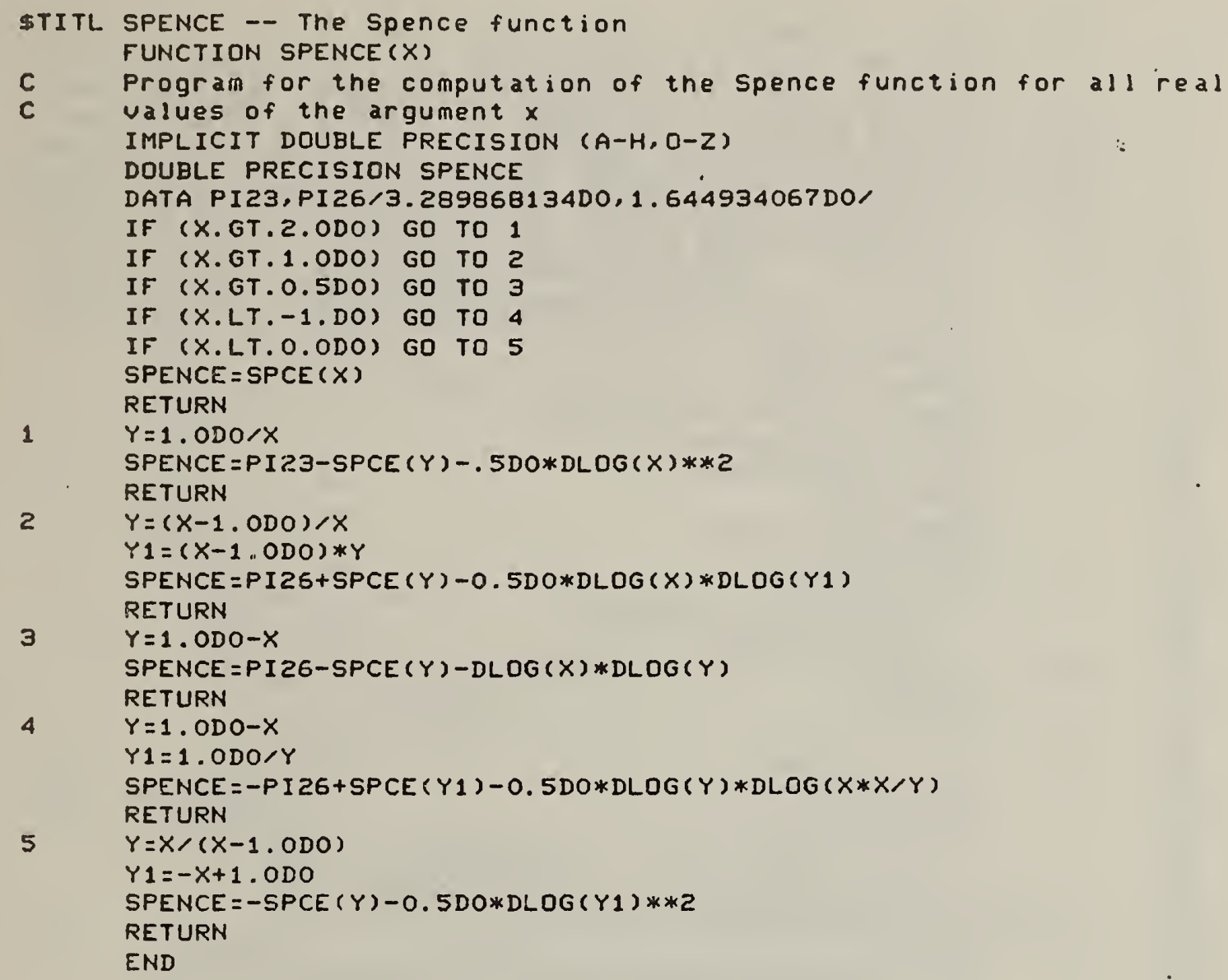


STITL EUALI -- Simpson's rule integration FUNCTION EUALI (A, B, FUNC, NS)

$C$ Function to evaluate the integral of the function FUNC

$C$ between $A$ and $B$. The number of steps $5 t a r t s$ at NSTEP

C as defined in EUALI and then goes up by a factor of two

C until the integral stops changing by more than a factor of EPS. In this version, which uses simpson's rule, the new values needed when NSTEP increases are not all recalculated. Ualues computed during the calculation of the old (inaccurate) value of the integral are re-used. IMPLICIT DOUBLE PRECISION $(A-H, O-Z)$

EXTERNAL FUNC DOUBLE PRECISION EUALI COMMON /EUICOM/ NSTEP, EPS

CALL FUNC (A,EUALA)

CALL F"UNC(B,EUALB)

NS $=$ NSTEP-1

STEPO $=(B-A) / N S T E P$

EUAL $2=0 . D O$

DO $900 \quad I=1, N S$

CALL FUNC $(A+I * S T E P O, U)$

soo

CONT INUE

c NS=NSTE.P

C

Start of $100 p$

STEP $=.5 D O *$ STEPO

EUAL $4=0 . D O$

DO $901 I=1$, NS

CALL FUNC $(A+S T E P+(I-1) * S T E P O, U)$

EUAL4 = EUAL $4+U$

901 CONT INUE

$E U=(E \cup A L A+2 . D O * E \cup A L 2+4 . D O * E \cup A L 4+E \cup A L B) * S T E P O / 6 . D O$ IF (NS.NE.NSTEP) THEN IF (EPS.LE.O.DO) THEN IF (DABS(EU-EUALIO).LE.DABS(EU*EPS)) GO TO 6

$C D$ WRITE $(1, *)$ A,B, DABS (EU-EUALIO), DABS(EU*EPS)

$C D$ ELSE

IF (DABS(EU-EUALIO).LE.EPS) GO TO 6

ENDIF ENDIF

WRITE $(1, *)$ A, B, DABS (EU-EUALIO), EPS

EUALID $=E U$

EUAL2 = EUAL4+EUAL2

$N S=2 * N S$

STEPO $=$ STEP

GO TO 4

$6 \quad$ EUALI $=E U$

RETURN

END 


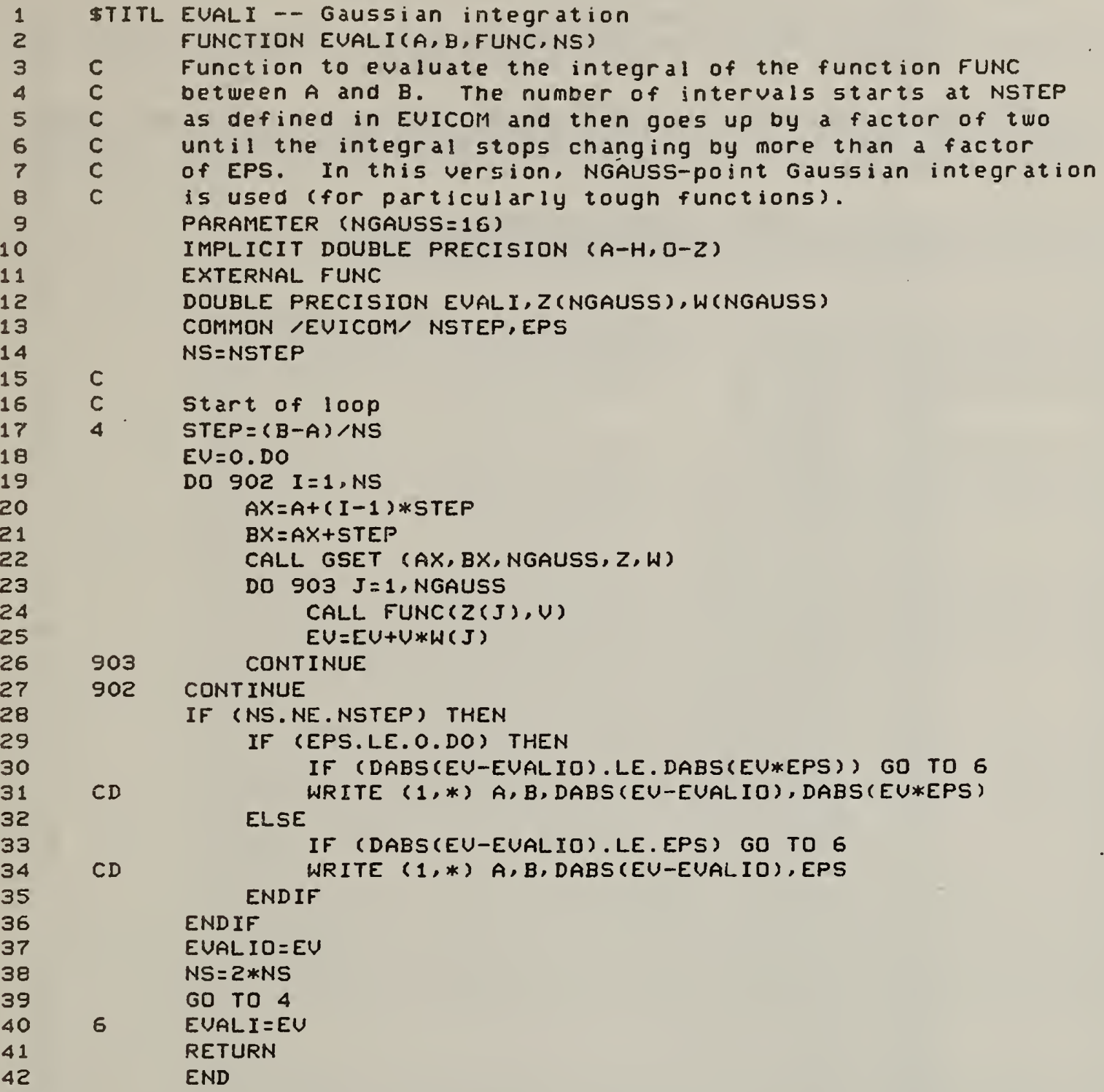


STITL GSET - - N-point GaUss zeros and weights SUBROUTINE GSET (AX,BX,NX,Z,W)

$C \quad N$-point Gauss zeros and weights for the interval ( $A x, B x$ ) are $C$ stored in arrays $z$ and $W$ respectively.

C This version converted from the version in the CERN I ibrary C for the CDC-7600 to a double precision version for a 32-bit C machine.

C

IMPLICIT DOUBLE PRECISION $(A-H, O-Z)$

DOUBLE PRECISION $Z(2), W(2), A(273), x(273)$

C INTEGER KTAB(96)

C Table of initial subscripts for $N=2(1) 16(4) 96$

DATA KTAB(2)/1/

DATA KTAB (3)/2/

DATA KTAB (4)/4/

DATA KTAB (5)/6,

DATA KTAB(6)/9/

DATA KTAB (T)/12/

DATA KTAB $(B) / 16 /$

DATA KTAB(9)/20/

DATA KTAB (10)/25/

DATA KTAB (11)/30/

DATA KTAB (12)/36/

DATA KTAB (13)/42/

DATA KTAB (14)/49/

DATA KTAB( 15$) / 56 /$

DATA KTAB (16)/64/

DATA KTAB (20)/72/

DATA KTAB(24)/82/

DATA KTAB (28)/82/

DATA KTAB (32)/94/

DATA KTAB(36)/94/

DATA KTAB $(40) / 110 /$

DATA KTAB( 44$) / 110 /$

DATA KTAB $(48) / 130 /$

DATA KTAB (52)/130/

DATA KTAB(56)/130/

DATA KTAB(60)/130/

DATA KTAB $(64) / 154 /$

DATA KTAB(68)/154/

DATA KTAB (72)/154/

DATA KTAB (76)/154/

DATA KTAB (80)/186/

DATA KTAB $(84) / 186 /$

DATA KTAB (88)/186/

DATA KTAB(92)/186/

C

DATA KTAB(96)/226/

C Table of abscissae ( $X)$ and weights (A) for interval $(-1,+1)$.

$\mathrm{C}$

$N=2$

DATA $X(1) / 0.577350269189626 D 0, A(1) / 1.000000000000000 D 0$

C

$N=3$

DATA $X(2) / 0.774596669241483 D 0 / A(2) / 0.555555555555556 D 0$

DATA $X(3) / 0.000000000000000 D 0, A(3) / 0.888888888888889 D 0$ 


\begin{tabular}{|c|c|c|c|c|c|}
\hline 58 & C & $N=4$ & & & \\
\hline 59 & & DATA & $x(4) / 0.86113631159405300$ & 1, & $A(4) / 0.347854845137454 D 0$ \\
\hline 60 & & DATA & $x(5) / 0.33998104358485600$ & , & $A(5) / 0.652145154862546 D 0$ \\
\hline 61 & C & $N=5$ & & & \\
\hline 62 & & DATA & $x(6) / 0.90617984593865400$ & , & $A(6) / 0.236926885056189 D 0$ \\
\hline 63 & & DATA & $x(7) / 0.53846931010568300$ & , & $A(7) / 0.47862867049936600$ \\
\hline 64 & & DATA & $x(8) / 0.00000000000000000$ & l, & $A(8) / 0.56888888888888900$ \\
\hline 65 & C & $N=6$ & & & \\
\hline 66 & & DATA & $x(9) / 0.93246951420315200$ & 1, & $A(9) / 0.17132449237917000$ \\
\hline 67 & & DATA & $x(10) / 0.66120938646626500$ & 1, & $A(10) / 0.36076157304813900$ \\
\hline 68 & & DATA & $x(11) / 0.23861918608319700$ & , & $A(11) / 0.467913934572691 \mathrm{DO}$ \\
\hline 69 & C & $N=7$ & & & \\
\hline 70 & & DATA & $x(12) / 0.94910791234275900$ & , & $A(12) / 0.12948496616887000$ \\
\hline 71 & & DATA & $x(13) / 0.741531185599394 \mathrm{DO}$ & , & $A(13) / 0.279705391489277 D 0$ \\
\hline 72 & & DATA & $x(14) / 0.40584515137739700$ & 1, & $A(14) / 0.38183005050511900$ \\
\hline 73 & & DATA & $x(15) / 0.00000000000000000$ & 1, & $A(15) / 0.417959183673469 D 0$ \\
\hline 74 & C & $N=8$ & & & \\
\hline 75 & & DATA & .96028985649753600 & 1, & 10.10122853629037600 \\
\hline 76 & & DATA & $x(17) / 0.79666647741362700$ & 1, & $A(17) / 0.222381034453374 D 0$ \\
\hline 77 & & DATA & $x(18) / 0.52553240991632900$ & , & $A(18) / 0.313706645877887 \mathrm{DO}$ \\
\hline 78 & & DATA & $x(19) / 0.18343464249565000$ & 1, & $A(19) / 0.36268378337836200$ \\
\hline 79 & C & $N=9$ & & & \\
\hline 80 & & DATA & $x(20) / 0.96816023950762600$ & 1, & $A(20) / 0.081274388361574 D 0$ \\
\hline 81 & & DATA & $x(21) / 0.83603110732663600$ & , & $A(21) / 0.180648160694857 D 0$ \\
\hline 82 & & DATA & $x(22) / 0.61337143270059000$ & 1, & $A(22) / 0.26061069640293500$ \\
\hline 83 & & DATA & $x(23) / 0.32425342340380900$ & , & $A(23) / 0.31234707704000300$ \\
\hline 84 & & DATA & $x(24) / 0.00000000000000000$ & , & $A(24) / 0.33023335500126000$ \\
\hline 85 & C & $N=10$ & & & \\
\hline 86 & & DATA & $x(25) / 0.973906528517172 D 0$ & ， & $A(25) / 0.06667134430868800$ \\
\hline 87 & & DATA & $x(26) / 0.86506335668898500$ & 1, & $A(26) / 0.149451349150581 D 0$ \\
\hline 88 & & DATA & $x(27) / 0.67940956829902400$ & , & $A(27) / 0.219086362515982 D 0$ \\
\hline 89 & & DATA & $x(28) / 0.433395394129247 \mathrm{DO}$ & , & $A(28) / 0.269266719309996 D 0$ \\
\hline 90 & & DATA & $x(29) / 0.14887433898163100$ & ， & $A(29) / 0.295524224714753 D 0$ \\
\hline 91 & c & $N=11$ & & & \\
\hline 92 & & DATA & $x(30) / 0.978228658146057 D 0$ & $\prime$, & $A(30) / 0.055668567116174 D 0$ \\
\hline 93 & & DATA & $x(31) / 0.88706259976809500$ & l, & $A(31) / 0.125580369464905 D 0$ \\
\hline 94 & & DRTA & .730 & l: & $0210927734 \mathrm{DO}$ \\
\hline 95 & & DATA & $x(33) / 0.51909612920681200$ & ， & $A(33) / 0.233193764591990 \mathrm{DO}$ \\
\hline 96 & & DATA & $x(34) / 0.26954315595234500$ & ， & $A(34) / 0.262804544510247 D 0$ \\
\hline 97 & & DATA & $x(35) / 0.00000000000000000$ & 1, & $A(35) / 0.27292508677790100$ \\
\hline 98 & c & $N=12$ & & & \\
\hline 99 & & DATA & 0.98156 & , & $A(36) / 0.047175$ \\
\hline 100 & & DATA & $\times(37) / 0.904$ & 八, & $A(37) / 0.10693932599531800$ \\
\hline 101 & & DATA & $x(38) / 0.76990267419430500$ & , & $A(38) / 0.16007832854334600$ \\
\hline 102 & & DATA & $x(39) / 0.58731795428661700$ & , & $A(39) / 0.20316742672306600$ \\
\hline 103 & & DATA & $x(40) / 0.367831498998180 D 0$ & , & $A(40) / 0.233492536538355 D 0$ \\
\hline 104 & & DATA & $x(41) / 0.12523340851146900$ & l, & $A(41) / 0.24914704581340300$ \\
\hline 105 & c & $N=13$ & & & \\
\hline 106 & & DATA & $x(42) / 0.984183054718588 D 0$ & l, & $A(42) / 0.040484004765316 D 0$ \\
\hline 107 & & DATA & $x(43) / 0.917598399222978 D 0$ & 1, & $A(43) / 0.092121499837728 D 0$ \\
\hline 108 & & DATA & $x(44) / 0.80157809073331000$ & , & $A(44) / 0.138873510219787 D 0$ \\
\hline 109 & & DATA & $x(45) / 0.642349339440340 D 0$ & 1, & $A(45) / 0.17814598076194600$ \\
\hline 110 & & DATA & $x(46) / 0.448492751036447 D 0$ & 1, & $A(46) / 0.20781604753688900$ \\
\hline 111 & & DATA & $x(47) / 0.230458315955135 \mathrm{DO}$ & , & $A(47) / 0.226283180262897 D 0$ \\
\hline 112 & & DATA & $x(48) / 0.00000000000000000$ & l, & $A(48) / 0.232551553230874 D 0$ \\
\hline 113 & C & $N=14$ & & & \\
\hline 114 & & DATA & $x(49) / 0.98628380869681200$ & l, & $A(49) / 0.035119460331752 D 0$ \\
\hline
\end{tabular}




\begin{tabular}{|c|c|c|c|c|c|c|}
\hline 115 & & DATA & $x(50) / 0.928434883663574 D 0$ & , & $A(50)=0.08015808715976000$ & , \\
\hline 116 & & DATA. & $x(51) / 0.82720131506976500$ & , & $A(51) / 0.121518570687903 D 0$ & , \\
\hline 117 & & DATA & $x(52) / 0.68729290481168500$ & , & $A(52) / 0.157203167158194 D 0$ & > \\
\hline 118 & & DATA & $x(53) / 0.515248636358154 D 0$ & , & $A(53) / 0.185538397477938 D 0$ & , \\
\hline 119 & & DATA & $x(54) / 0.31911236892789000$ & $\mu$ & $A(54) / 0.205198463721296 D 0$ & , \\
\hline 120 & & DATA & $x(55)<0.10805494870734400$ & 1, & $A(55) / 0.215263853463158 D 0$ & ノ \\
\hline 121 & C & $N=15$ & & & & \\
\hline 122 & & DATA & $x(56) / 0.98799251802048500$ & 1, & $A(56) / 0.030753241996117 D 0$ & < \\
\hline 123 & & DATA & $x(57) / 0.93727339240070600$ & 1, & $A(57) / 0.07036604748810800$ & , \\
\hline 124 & & DATA & $x(58) / 0.84820658341042700$ & 1, & $A(58) / 0.107159220467172 D 0$ & , \\
\hline 125 & & DATA & $x(59) / 0.72441773136017000$ & 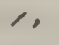 & $A(59) / 0.139570677926154 D 0$ & , \\
\hline 126 & & DATA & $x(60) / 0.57097217260853900$ & 八, & $A(60) / 0.166269205816994 D 0$ & , \\
\hline 127 & & DATA & $x(61) / 0.39415134707756300$ & 1, & $A(61) / 0.186161000015562 D 0$ & 子 \\
\hline 128 & & DATA & $x(62) / 0.20119409399743500$ & , & $A(62) / 0.198431485327111 D 0$ & , \\
\hline 129 & & DATA & $x(63) / 0.00000000000000000$ & 1, & $A(63) / 0.202578241925561 D 0$ & , \\
\hline 130 & C & $N=16$ & & & & \\
\hline 131 & & DATA & $x(64) / 0.98940093499165000$ & 八 & $A(64) / 0.027152459411754 D 0$ & , \\
\hline 132 & & DATA & $x(65) / 0.94457502307323300$ & , & $A(65)>0.062253523938648 D 0$ & , \\
\hline 133 & & DATA & $x(66) / 0.865631202387832 D 0$ & , & $A(66) / 0.09515851168249300$ & , \\
\hline 134 & & DATA & $x(67) / 0.75540440835500300$ & 八, & $A(67) / 0.124628971255534 D 0$ & , \\
\hline 135 & & DATA & $x(68) / 0.617876244402644 D 0$ & 八, & $A(68) / 0.149595988816577 D 0$ & ' \\
\hline 136 & & DATA & $x(69) / 0.458016777657227 D 0$ & 1, & $A(69) / 0.16915651939500300$ & > \\
\hline 137 & & DATA & $x(70) / 0.28160355077925900$ & , & $A(70) / 0.182603415044924 D 0$ & , \\
\hline 138 & & DATA & $x(71) / 0.09501250983763700$ & 1, & $A(71) / 0.18945061045506900$ & , \\
\hline 139 & c & $N=20$ & & & & \\
\hline 140 & & DATA & $x(72) / 0.993128599185094 \mathrm{DO}$ & 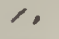 & $A(72) / 0.017614007139152 D 0$ & ' \\
\hline 141 & & DATA & $x(73) / 0.96397192727791300$ & 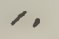 & $A(73) / 0.040601429800386 D 0$ & r \\
\hline 142 & & DATA & $x(74) / 0.91223442825132500$ & 1, & $A(74) / 0.06267204833410900$ & , \\
\hline 143 & & DATA & $x(75) / 0.83911697182221800$ & , & $A(75) / 0.083276741576704 D 0$ & , \\
\hline 144 & & DATA & $x(76) / 0.74633190646015000$ & , & $A(76) / 0.101930119817240 D 0$ & , \\
\hline 145 & & DATA & $x(77) / 0.63605368072651500$ & , & $A(77) / 0.118194531961518 D 0$ & , \\
\hline 146 & & DATA & $x(79) / 0.51086700195082700$ & $\prime$ & $A(78) / 0.131688638449176 D 0$ & ' \\
\hline 147 & & DATA & $x(79) / 0.37370608871541900$ & , & $A(79) / 0.142096109318382 D 0$ & , \\
\hline 148 & & DATA & $x(80) / 0.22778585114164500$ & 1. & $A(80) / 0.149172986472603 D 0$ & , \\
\hline 149 & & DATA & $x(81) / 0.076526521133497 \mathrm{DO}$ & $\prime$, & $A(81) / 0.152753387130725 D 0$ & ' \\
\hline 150 & C & $N=24$ & & & & \\
\hline 151 & & DATA & $x(82) / 0.995187219997021 D 0$ & l, & $A(82) / 0.012341229799987 D 0$ & ' \\
\hline 152 & & DATA & $x(83) / 0.97472855597130900$ & $\prime$, & $A(83) / 0.028531388628933 D 0$ & ' \\
\hline 153 & & DATA & $x(84) / 0.938274552002732 D 0$ & 1, & $A(84) / 0.04427743881741900$ & , \\
\hline 154 & & DATA & $x(85) / 0.88641552700440100$ & 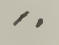 & $A(85) / 0.059298584915436 D 0$ & ' \\
\hline 155 & & DATA & $x(86) / 0.82000198597390200$ & , & $A(86) / 0.073346481411080 D 0$ & r \\
\hline 156 & & DATA & $x(87) / 0.74012419157855400$ & ， & $A(87) / 0.08619016153195300$ & ' \\
\hline 157 & & DATA & $x(88) / 0.64809365193697500$ & $\prime$ & $A(88) / 0.097618652104113 D 0$ & ' \\
\hline 158 & & DATA & $x(89) / 0.54542147138883900$ & 八, & $A(89) / 0.10744427011596500$ & , \\
\hline 159 & & DATA & $x(90) / 0.43379350762604500$ & , & $A(90) / 0.115505668053725 D 0$ & , \\
\hline 160 & & DATA & $x(91) / 0.31504267969616300$ & $\prime$, & $A(91) / 0.121670472927803 D 0$ & ' \\
\hline 161 & & DATA & $x(92) / 0.19111886747361600$ & , & $A(92) / 0.125837456346828 D 0$ & , \\
\hline 162 & & $\begin{array}{l}\text { DATA } \\
N-3=?\end{array}$ & $x(93) / 0.06405689286260500$ & 八, & $A(93) / 0.127938195346752 D 0$ & ' \\
\hline 163 & c & $N=32$ & & & & \\
\hline 164 & & DATA & $x(94) / 0.997263861849481 D 0$ & 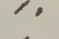 & $A(94) / 0.007018610009470 D 0$ & ' \\
\hline 165 & & DATA & $x(95) / 0.985611511545268 D 0$ & 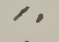 & $A(95) / 0.016274394730905 D 0$ & ' \\
\hline 166 & & DATA & $x(96) / 0.96476225558750600$ & 八 & $A(96) / 0.025392065309262 D 0$ & ' \\
\hline 167 & & DATA & $x(97) / 0.93490607593773900$ & 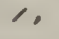 & $A(97) / 0.034273862913021 \mathrm{DO}$ & ' \\
\hline 168 & & DATA & $x(98) / 0.896321155766052 D 0$ & 八, & $A(98) / 0.042835898022226 D 0$ & , \\
\hline 169 & & DATA & $x(99) / 0.84936761373256900$ & 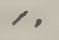 & $A(99) / 0.050998059262376 D 0$ & 八 \\
\hline 170 & & DATA & $x(100) / 0.79448379596794200$ & , & $A(100) / 0.05868409347853500$ & \\
\hline 171 & & DATA & $x(101) / 0.73218211874028900$ & , & $A(101) / 0.065822222776361 D 0$ & \\
\hline
\end{tabular}


DATA $X(102) / 0.663044266930215 \mathrm{DO} /$, DATA $X(103) / 0.587715757240762 \mathrm{DO} /$, DATA $\times(104) / 0.506899908932229 D 0 /$, DATA $X(105) / 0.421351276130635 D 0 /$, DATA $X(106) / 0.331868602282127 \mathrm{DO} /$, DATA $\times(107) / 0.23928736225213700 /$, DATA $X(108) / 0.144471961582796 \mathrm{DO} /$, DATA $X(109) / 0.048307665687738 D 0 /$, $N=40$

DATA $X(110) / 0.998237709710559 D 0 /$, DATA $X(111) / 0.990726238699457 \mathrm{DO} /$, DATA $X(112) / 0.977259949983774 D 0 /$, DATA $X(113) / 0.957916819213791 \mathrm{DO} /$, DATA $X(114) / 0.932812808278676 \mathrm{DO} /$, DATA $X(115) / 0.902098806968874 D 0 /$, DATA $X(116) / 0.865959503212259 D 0 /$, DATA $\times(117) / 0.824612230833311 \mathrm{DO} /$, DATA $X(118) / 0.778305651426519 D 0 /$, DATA $X(1.19) / 0.727318255189927 \mathrm{DO} /$, DATA $\times(120) / 0.671956684614179 D 0 /$, DATA $\times(121) / 0.612553889667980 D 0 /$, DATA $X(122) / 0.54946712509512900 /$, DATA $X(123) / 0.483075801686178 D 0 /$, DATA $X(124) / 0.41377920437160500 /$, DATA $X(125) / 0.341994090825758 D 0 /$, DATA $\times(126) / 0.268152185007253 D 0 /$, DATA $X(127) / 0.19269758070137100 /$, DATA $x(128) / 0.116084070675255 \mathrm{DO} /$, DATA $X(129) / 0.038772417506050 D 0 /$, $N=4 B$

DATA $X(130) / 0.998771007252426 \mathrm{DO} /$, DATA $X(131) / 0.993530172266350 D 0 /$, DATA $\times(132) / 0.984124583722826 \mathrm{DO} /$, DATA $\times(133) / 0.970591592546247 \mathrm{DO} /$ DATA $\times(134) / 0.952987703160430 \mathrm{DO} /$, DATA $x(135) / 0.931386690706554 \mathrm{DO} /$ DATA $X(136) / 0.905879136715569 \mathrm{DO} /$, DATA $\times(137) / 0.876572020274247 \mathrm{DO} /$, DATA $X(138) / 0.843588261624393 \mathrm{DO} /$, DATA $X(139) / 0.807066204029442 D 0 /$, DATA $X(140) / 0.767159032515740 D 0 /$, DATA $\times(141) / 0.724034130923814 D 0 /$, DATA $X(142) / 0.677872379632663 D 0 /$, DATA $x(143) / 0.628867396776513 \mathrm{DO} /$, DATA $X(144) / 0.577224726083972 D 0 /$, DATA $\times(145) / 0.523160974722233 D 0 /$, DATA $x(146) / 0.466902904750958 D 0 /$, DATA $X(147) / 0.408686481990716 \mathrm{DO} /$ DATA $\times(148) / 0.348755886292160 D 0 /$, DATA $X(149) / 0.28736248735545500 /$, DATA $X(150) / 0.224763790394689 D 0 /$, DATA $x(151) / 0.161222356068891 \mathrm{DO} /$, DATA $x(152) / 0.097004699209462 \mathrm{DO} /$, DATA $X(153) / 0.0323801$ ? $096286900 /$, $N=64$

DATA $X(154) / 0.999305041735772 D 0 /$, DATA $X(155) / 0.996340116771955 \mathrm{DO} /$,
$A(102) / 0.072345794108848 D 0 /$ $A(103) / 0.07819389578707040 /$ $A(104) / 0.083311924226946 D 0 /$ $A(105) / 0.087652093004403 D 0 /$ $A(106) / 0.091173878695763 D 0 /$ $A(107) / 0.093844399080804 D 0 /$ $A(108) / 0.095638720079274 D O /$ $A(109) / 0.096540088514727 D O$, $A(110) / 0.004521277098533 D 0 /$ $A(111) / 0.010498284531152 \mathrm{DO} /$ $A(112) / 0.016421058381907 D O /$ $A(113) / 0.022245849194166 \mathrm{DO} /$ $A(114) / 0.027937006980023 D 0 /$ $A(115) / 0.033460195282547 D O /$ $A(116) / 0.038782167974472 \mathrm{DO} /$ $A(117) / 0.043870908185673 D 0 /$ $A(118) / 0.048695807635072 D 0 /$ $A(119) / 0.05322784698393600 /$ $A(120) / 0.057439769099391 \mathrm{DO} /$ $A(121) / 0.061306242492928 D 0 /$ $A(122) / 0.064804013456601 \mathrm{DO} /$ $A(123) / 0.067912045815233 D 0 /$ $A(124) / 0.070611647391286 \mathrm{DO} /$ $A(125) / 0.072886582395804 D O /$ $A(126) / 0.074723169057968 D 0 /$ $A(127) / 0.076110361900626 \mathrm{DO} /$ $A(128) / 0.077039818164247 \mathrm{DO} /$ $A(129) / 0.077505947978424 D 0 /$ $A(130) / 0.003153346052305 \mathrm{DO} /$ $A(131) / 0.007327553901276 \mathrm{DO} /$ $A(132) / 0.011477234579234 D O /$ $A(133) / 0.015579315722943 D 0 /$ $A(134) / 0.019616160457355 \mathrm{DO} /$ $A(135) / 0.023570760839324 D O /$ $A(136) / 0.027426509708356 D 0 /$ $A(137) / 0.031167227832798 D 0 /$ $A(138) / 0.034777222564770 D O /$ $A(139) / 0.038241351065830 D 0 /$ $A(140) / 0.041545082543464 D O /$ $A(141) / 0.044674560856694 \mathrm{DO} /$ $A(142) / 0.047616658492490 D 0 /$ $A(143) / 0.050359035553854 \mathrm{DO} /$ $A(144) / 0.052890189485193 D 0 /$ $A(145) / 0.055199503699984 D O /$ $A(146) / 0.057277292100403 D O /$ $A(147) / 0.059114839698395 D O /$ $A(148) / 0.060704439165893 D O /$ $A(149) / 0.062039423159892 D O /$ $A(150) / 0.063114192286254 D 0 /$ $A(151) / 0.063924238584648 D 0 /$ $A(152) / 0.054466164435950 \mathrm{DO} /$ $A(153) / 0.064737696812683 D 0 /$ $A(154) / 0.001783280721696 \mathrm{DO} /$ $A(155) / 0.004147033260562 \mathrm{DO} /$ 
229

230

231

232

233

234

235

236

237

238

239

240

241

242

243

244

245

246

247

248

249

250

251

252

253

254

255

256

257

258

259

260

261

262

263

264

265

266

267

268

259

270

271

272

273

274

275

276

277

278

279

280

281

282

283

284

285
DATA $\times(156) / 0.991013371476744 D 0 /$, DATA $X(157) / 0.983336253884625 D 0 /$, DATA $X(158) / 0.973326827789910 D 0 /$, DATA $\times(159) / 0.961008799652053 D 0 \%$ DATA $X(160) / 0.946411374858402 D 0 /$, DATA $X(161) / 0.929569172131939 D 0 /$, DATA $x(162) / 0.910522137078502 D 0 /$, DATA $X(163) / 0.889315445995114 \mathrm{DO} /$, DATA $X(164) / 0.865999398154092 \mathrm{DO} /$, DATA $X(165) / 0.840629296252580 D 0 \%$, DATA $X(166) / 0.813265315122797 D 0 /$, DATA $x(167) / 0.783972358943341 \mathrm{DO} /$ DATA $X(168) / 0.752819907260531 \mathrm{DO} /$, DATA $X(169) / 0.719881850171610 D 0 /$, DATA $X(170) / 0.685236313054233 D 0 /$, DATA $X(171) / 0.64896547125465700 /$, DATA $x(172) / 0.611155355172393 D 0 /$, DATA $X(173) / 0.571895646202634 D 0 /$, DATA $X(174) / 0.531279464019894 D 0 /$, DATA $\times(175) / 0.489403145707052 D 0 /$, DATA $X(176) / 0.446366017253464 \mathrm{DO} /$, DATA $X(177) / 0.402270157963991 \mathrm{DO} /$, DATA $X(178) / 0.357220158337668 D 0 /$, DATA $X(179) / 0.311322871990210 D 0 /$, DATA $X(180) / 0.264687162208767 D 0 /$, DATA $X(181) / 0.217423643740007 D 0 /$, DATA $X(182) / 0.169644420423992 D 0 /$, DATA $\times(183) / 0.121462819296120 D 0 /$, DATA $X(184) / 0.07299312178779900 /$, DATA $X(185) / 0.024350292663424 D 0 /$, $N=80$

DATA $X(186) / 0.999553822651630 D 0 /$, DATA $X(187) / 0.997645864398237 D 0 /$, DATA $\times(168) / 0.994227540965688 D 0 /$, DATA $\times(189) / 0.989291302499755 D 0 /$, DATA $\times(190) / 0.982848572738629 D 0 /$, DATA $\times(191) / 0.974909140585727 D 0 /$, DATA $\times(192) / 0.965485089043799 D 0 /$, DATA $\times(193) / 0.954590766343634 \mathrm{DO} /$, DATA $\times(194) / 0.942242761309872 D 0 /$ DATA $X(195) / 0.928459877172445 D 0 /$, DATA $\times(196) / 0.913263102571757 D 0 /$, DATA $\times(197) / 0.896675579438770 D 0 /$, DATA $X(198) / 0.87872256767821300 /$, DATA $X(199) / 0.859431406663111 \mathrm{DO} /$ DATA $\times(200) / 0.838831473580255 D 0 /$, DATA $X(201) / 0.816954138681463 D 0 /$, DATA $\times(202) / 0.793832717504605 D 0 /$, DATA $X(203) / 0.769502420135041 \mathrm{DO} /$ DATA $\times(204) / 0.744000297583597 D 0 /$, DATA $\times(205) / 0.717365185362099 D 0 /$, DATA $X(206) / 0.689637544342027 D 0 /$ DATA $\times(207) / 0.66085989898611900 /$, DATA $\times(208) / 0.631075773046871 \mathrm{DO} /$, DATA $X(209) / 0.600330622829751 \mathrm{DO} /$, DATA $X(210) / 0.568671268122709 D 0 /$, DATA $\times(211) / 0.536145920897131 \mathrm{DO} /$
$A(156) / 0.006504457968978 D 0 /$ $A(157) / 0.008846759826363 D 0 /$ $A(158) / 0.011168139460131 \mathrm{DO} /$ $A(159) / 0.013463047896718 D 0 /$ $A(160) / 0.015726030476024 D 0 /$ $A(161) / 0.017951715775697 \mathrm{DO} /$ $A(162) / 0.020134823153530 D 0 /$ $A(163) / 0.02227017380838300 /$ $A(164) / 0.024352702568710 D 0 /$ $A(165) / 0.026377469715054 D 0 /$ $A(166) / 0.028339672614259 D 0 /$ $A(167) / 0.030234657072402 D 0 /$ $A(168) / 0.032057928354851 \mathrm{DO} /$ $A(169) / 0.033805161837141 \mathrm{DO} /$ $A(170) / 0.035472213256882 D O$ $A(171) / 0.037055128540240 D 0 /$ $A(172) / 0.038550153178615 D 0 /$ $A(173) / 0.039953741132720 D 0$ $A(174) / 0.041262563242623 D 0 /$ $A(175) / 0.042473515123653 D 0 /$ $A(176) / 0.043583724529323 D 0 /$ $A(177) / 0.044590558163756 \mathrm{DO} /$ $A(178) / 0.045491627927418 D 0 /$ $A(179) / 0.046284796581314 \mathrm{DO} /$ $A(180) / 0.046968182816210 D D /$ $A(181) / 0.047540165714830 D 0 /$ $A(182) / 0.047999388596458 D 0 /$ $A(183) / 0.048344762234802 \mathrm{DO} /$ $A(184) / 0.048575467441503 D 0 /$ $A(185) / 0.048690957009139 D 0 /$ $A(186) / 0.001144950003186 \mathrm{DO} /$ $A(187) / 0.002663533589512 D 0 /$ $A(188) / 0.004180313124694 \mathrm{DO} /$ $A(189) / 0.005690922451403 D 0 /$ $A(190) / 0.007192904768117 \mathrm{DO}$ $A(191) / 0.008683945269260 D 0 /$ $A(192) / 0.010161766041103 D 0 /$ $A(193) / 0.011624114120797 D 0 /$ $A(194) / 0.013068761592401 D 0 /$ $A(195) / 0.014493508040509 D 0 /$ $A(196) / 0.015896183583725 D O /$ $A(197) / 0.017274652056269 \mathrm{DO} /$ $A(198) / 0.018626814208299 D 0 /$ $A(199) / 0.019950610878141 D 0 /$ $A(200) / 0.021244026115782 D O /$ $A(201) / 0.022505090246332 D 0 /$ $A(202) / 0.023731882865930 D 0 /$ $A(203) / 0.024922535764115 \mathrm{DO}$ $A(204) / 0.026075235767565 D 0 /$ $A(205) 10.027188227500486 D 0 /$ $A(206) / 0.028259816057276 D 0 /$ $A(207) / 0.029288369583267 D 0 /$ $A(208) / 0.030272321759557 D 0$ $A(209) / 0.031210174188114 \mathrm{DO} /$ $A(210) / 0.032100498673487 D 0 /$ $A(211) / 0.032941939397645 D 0 /$ 
286

287

288

289

290

291

292

293

294

295

296

297

298

299

300

301

302

303

304

305

306

307

308

309

310

311

312

313

314

315

316

317

318

319

320

321

322

323

324

325

326

327

328

329

330

331

332

333

334

335

336

337

338

339

340

341

342
DATA $X(212) / 0.502804111888784 \mathrm{DO} /$, DATA $\times(213) / 0.468696615170544 D 0 /$, DATA $X(214) / 0.43387537083175600 /$, DATA $X(215) / 0.39839340588196900 /$, DATA $X(216) / 0.362304753499487 D 0 /$, DATA $X(217) / 0.32566437074770100 /$, DATA $X(218) / 0.288528054884511 \mathrm{DO} /$, DATA $X(219) / 0.250952358392272 D 0 /$, DATA $X(220) / 0.21299450285766600 /$, DATA $X(221) / 0.174712291832646 D 0 /$, DATA $\times(222) / 0.136164022809143 D 0 /$, DATA $X(223) / 0.097408398441584 \mathrm{DO} /$, DATA $\times(224) / 0.058504437152420 D 0 /$, DATA $X(225) / 0.01951138325679300 /$, $N=96$

DATA $\times(226) / 0.999689503883230 \mathrm{DO} /$, DATA $X(227) / 0.998364375863181 \mathrm{DO} /$, DATA $X(228) / 0.995981842987209 D 0 /$, DATA $X(229) / 0.992543900323762 D 0 /$, DATA $X(230) / 0.988054126329623 D 0 /$, DATA $X(231) / 0.98251 .7263563014 \mathrm{DO} /$, DATA $\times(232) / 0.975939174585136 \mathrm{DO} /$, DATA $\times(233) / 0.968326828463264 \mathrm{DO} /$, DATA $X(234) / 0.959688291448742 D 0 /$ DATA $X(235) / 0.950032717784437 D 0 /$, DATA $X(236) / 0.93937033975275500 \%$ DATA $\times(237) / 0.927712456722308 D 0 / 。$ DATA $X(238) / 0.915071423120898 D 0 /$, DATA $X(239) / 0.901460635315852 \mathrm{DO} /$, DATA $X(240) / 0.88689451740242000 /$, DATA $\times(241) / 0.871388505909296 \mathrm{DO} /$, DATA $X(242) / 0.854959033434601 \mathrm{DO} /$, DATA $X(243) / 0.837623511228187 \mathrm{DO} /$, DATA $\times(244) / 0.819400310737931 \mathrm{DO} /$, DATA $\times(245) / 0.800308744139140 D 0 /$ DATA $\times(246) / 0.780369043867433 D 0 /$, DATA $X(247) / 0.759602341176647 D 0 \%$, DATA $X(248) / 0.738030643744400 D 0 /$, DATA $X(249) / 0.715676812348967 D 0 /$, DATA $X(250) / 0.692564536642171 \mathrm{DO} /$, DATA $X(251) / 0.668718310043916 \mathrm{DO} /$, DATA $X(252) / 0.644163403784567 \mathrm{DO} /$ DATA $X(253) / 0.618925840125468 D 0 /$, DATA $X(254) / 0.593032364777572 \mathrm{DO} /$ DATA $\times(255) / 0.566510418561397 \mathrm{DO} /$, DATA $X(256) / 0.539388108324357 \mathrm{DO} /$, DATA $X(257) / 0.511694177154667 \mathrm{DO} /$ DATA $X(258) / 0.483457973920596 \mathrm{DO} /$ DATA $X(259) / 0.454709422167743 D 0 /$, DATA $X(260) / 0.425478988407300 D 0 /$, DATA $X(261) / 0.395797649828908 D O /$, DATA $X(262) / 0.365696861472313 \mathrm{DO} /$, DATA $\times(263) / 0.335208522892625 \mathrm{DO} /$, DATA $X(264) / 0.304364944354496 D 0 /$, DATA $\times(265) / 0.273198812591049 D 0 /$, DATA $X(266) / 0.241743156163840 D 0 /$ DATA $x(267) / 0.210031310460567 \mathrm{DO} /$,
$A(212) / 0.033733214984611 \mathrm{DO} /$ $A(213) / 0.034473120451753 D 0 /$ $A(214) / 0.035160529044747 D 0$, $A(215) / 0.035794393953416 D 0 /$ $A(216) / 0.036373749905835 D 0 /$ $A(217) / 0.036897714638276 D O /$ $A(218) / 0.037365490238730 D 0 /$ $A(219) / 0.037776364362001 \mathrm{DO} /$ $A(220) / 0.038129711314477 D O /$ $A(221) / 0.038424993006959 D 0 /$ $A(222) / 0.038661759774076 \mathrm{DO} /$ $A(223) / 0.038839651059051 \mathrm{DO} /$ $A(224) / 0.03895839596276900 /$ $A(225) / 0.039017813656306 \mathrm{DO} /$

$A(226) / 0.000796792065552 D O /$ $A(227) / 0.001853960788946 \mathrm{DO}$, $A(228) / 0.002910731817934 D O$, $A(229) / 0.003964554338444 D O /$ $A(230) / 0.005014202742927 D 0 /$ $A(231) / 0.006058545504235 D 0 /$ $A(232) / 0.007096470791153 \mathrm{DO} /$ $A(233) / 0.008126876925698 D 0 /$ $A(234) / 0.009148671230783 D 0 /$ $A(235) / 0.010160770535008 D 0 /$ $A(236) / 0.011162102099838 D 0 /$ $A(237) / 0.012151604671088 D 0 /$ $A(238) / 0.013128229566961 \mathrm{DO} /$ $A(239) / 0.014090941772314 \mathrm{DO} /$ $A(240) / 0.015038721026994 \mathrm{DO} /$ $A(241) / 0.015970562902562 D 0 /$ $A(242) / 0.016885479864245 D 0$, $A(243) / 0.017782502316045 D O /$ $A(244) / 0.018660679627411 \mathrm{DO} /$ $A(245) / 0.019519081140145 \mathrm{DO} /$ $A(246) / 0.020356797154333 D 0 /$ $A(247) / 0.021172939892191 \mathrm{DO} /$ $A(248) / 0.021966644438744 \mathrm{DO} /$ $A(249) / 0.022737069658329 D 0 /$ $A(250) / 0.023483399085926 \mathrm{DO} /$ $A(251) / 0.024204841792364 \mathrm{DO} /$ $A(252) / 0.024900633222483 D 0 /$ $A(253) / 0.02557003600534900$ $A(254) / 0.026212340735672 D 0 /$ $A(255) / 0.026826866725591 \mathrm{DO} /$ $A(256) / 0.02741296272602900 /$ $A(257) / 0.027970007616848 D 0 /$ $A(258) / 0.028497411055085 D 0 /$ $A(259) / 0.028994614150555 D 0 /$ $A(260) / 0.029461089958167 \mathrm{DO} /$ $A(261) / 0.029896344136328 D 0 /$ $A(262) / 0.030299915420827 D 0 /$ $A(263) / 0.0306713761236690 D /$ $A(264) / 0.031010332586313 D 0 /$ $A(265) / 0.031316425596861 \mathrm{DO} /$ $A(266) / 0.031589330770727 \mathrm{DO}$ $A(267) / 0.031828758894411 \mathrm{DO} /$ 


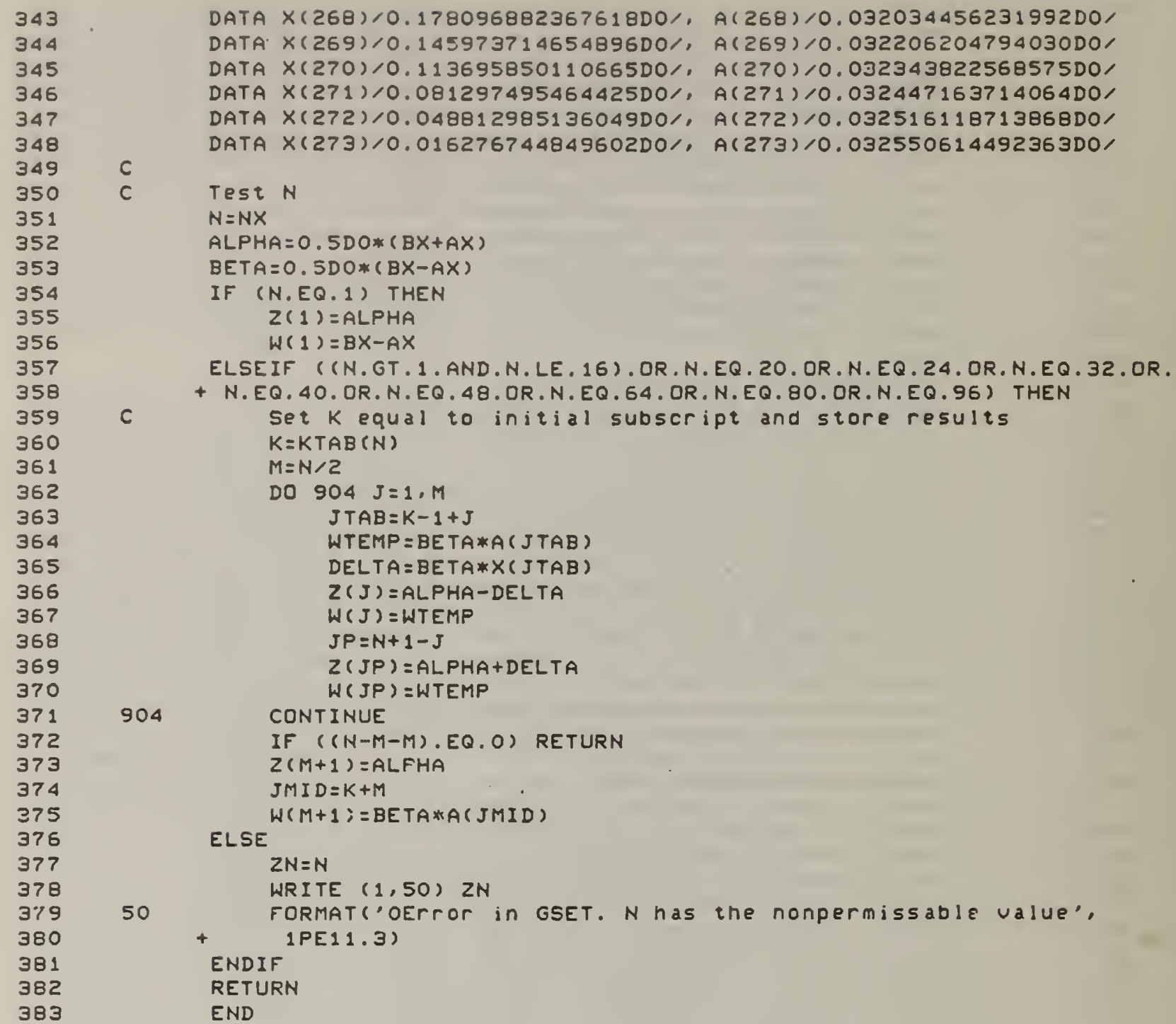




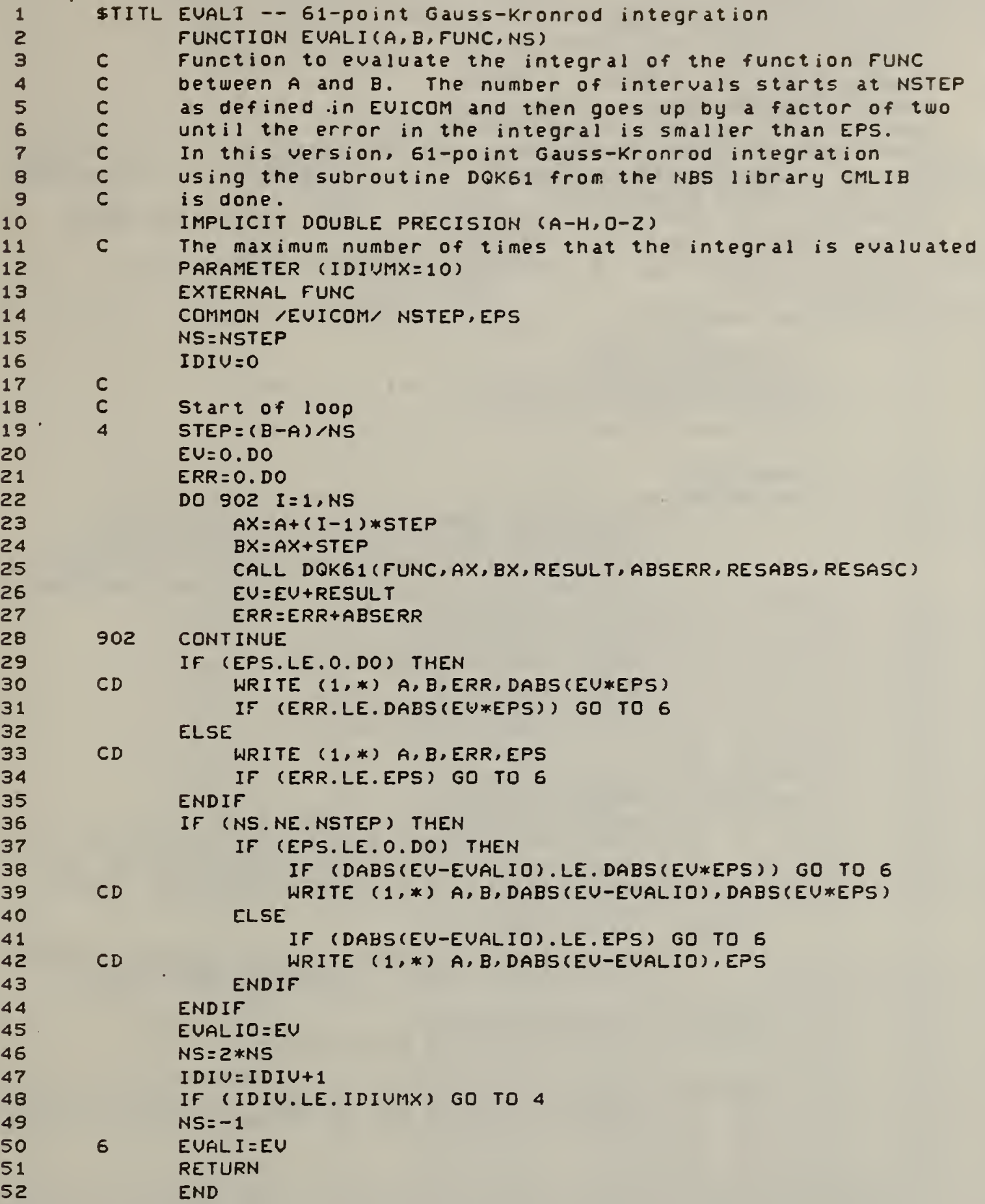




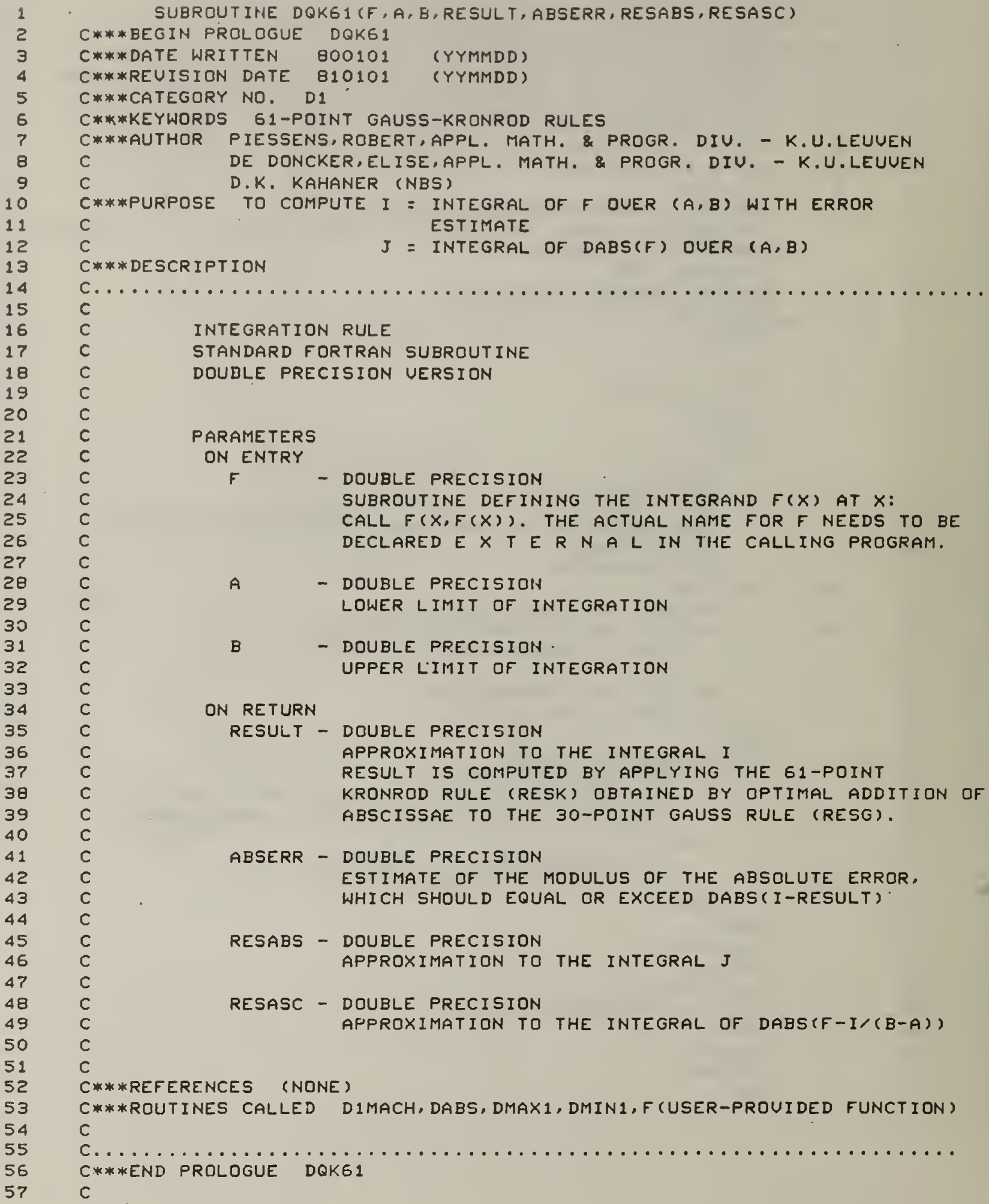




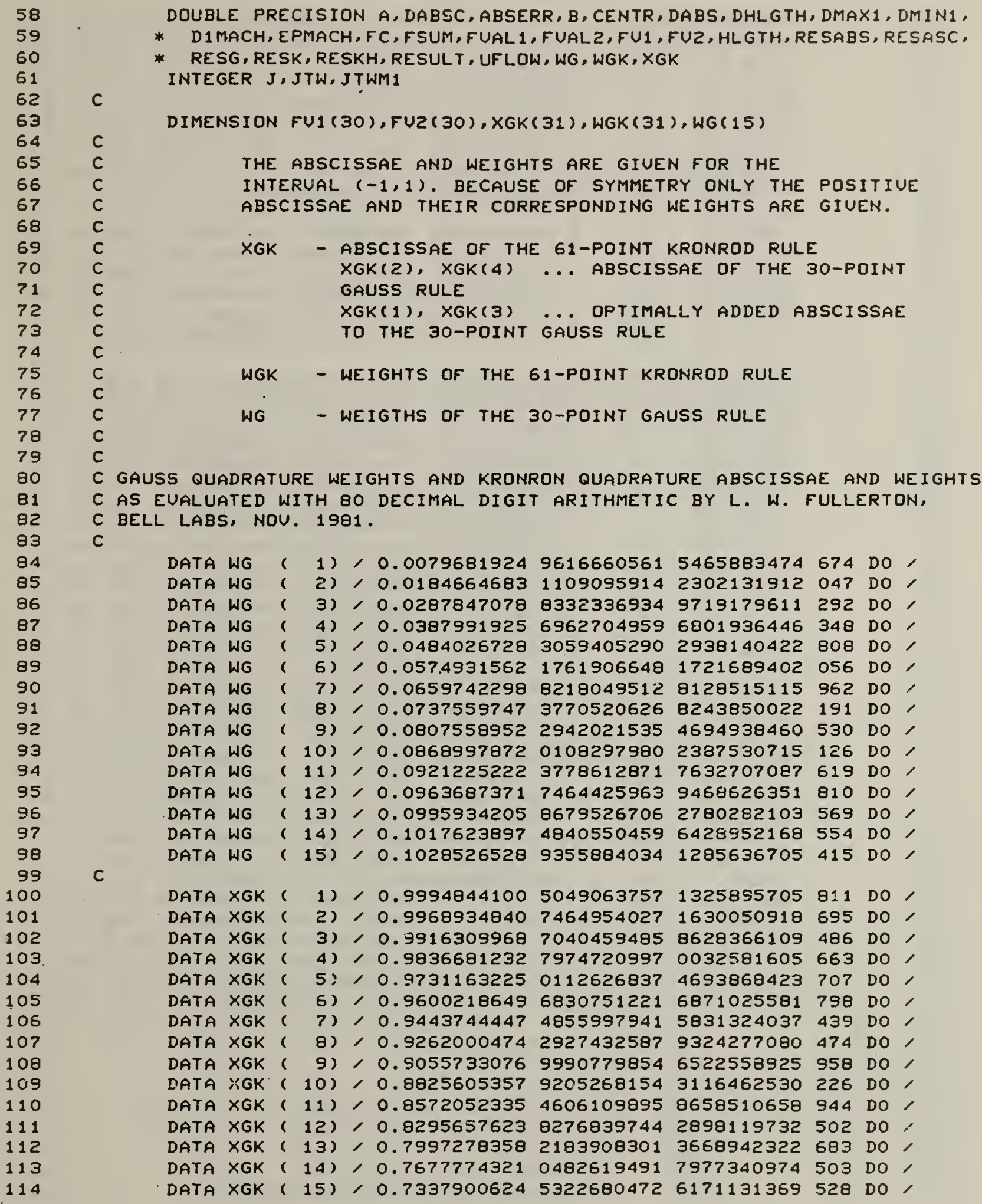


$c$

DATA XGK (16) , 0.697850494793315796932292388026 640 DO, DATA XGK ( 17) , 0.660061064126626961370053668149271 DO, DATA XGK (18) / 0.620526182989242861140477556431189 DO/ DATA XGK ( 19) / 0.579345235826361691756024932172540 DO DATA XGK ( 20) ; 0.53662414814201989926 4169793311 073 DO DATA XGK ( 21) , 0.4924804678 61778574993693061207 709 DO DATA XGK ( 22) / 0.447033769538089176780609900322854 DO DATA XGK ( 23) / 0.400401254830394392535476211542661 DO DATA XGK ( 24) , 0.35270472553087811347 1037207089 374 DO DATA XGK ( 25) , 0.3040732022 7362507737 2677107199 257 DO DATA XGK ( 26) , 0.254636926167889846439805129817805 DO/ DATA XGK (27) , 0.204525116682309891438957671002 025 DO/ DATA XGK (28) , 0.1538699136 0858354696 3794672743 256 DO DATA XGK ( 29), 0.1028069379 66737030147096751318001 DO/ DATA XGK ( 30$), 0.051471842555317695833025213166723$ DO , DATA XGK ( 31), 0.000000000000000000000000000000000 DO ,

DATA WGK ( 1) / 0.00138901369867700762 4551591226760 DO / DATA WGK (2) / 0.003890461127099884051267201844516 DO, DATA WGK ( 3), 0.006630703915931292173319826369750 DO, DATA WGK ( 4) / 0.0092732796 59517763428441146892024 DO , DATA WGK ( 5$) / 0.011823015253496341742232898853251$ DO / DATA WGK ( 6) , 0.0143697295 07045804812451432443580 DO, DATA WGK ( 7) / 0.016920889189053272627572289420322 DO, DATA WGK ( 8) $/ 0.019414141193942381173408951050128$ DO, DATA WGK ( 9) , 0.0218280358 21609192297167485738339 DO, DATA WGK (10), 0.024191162078080601365686370725 232 DO, DATA WGK (11), 0.0265099548 82333101610601709335075 DO , DATA WGK (12) / 0.028754048765041292843978785354334 DO, DATA WGK (13) , 0.0309072575 62387762472884252943092 DO, DATA WGK ( 14) , 0.0329814470 57483726031814191016854 DO, DATA WGK ( 15), 0.0349793380 28060024137499670731468 DO, DATA WGK (16) / 0.03688236465182122922 3911065617136 DO, DATA WGK (17), 0.0386789456 2472759295 0348651532281 DO, DATA WGK (18) / 0.040374538951535959111995279752468 DO , DATA WGK (19) / 0.041969810215164246147147541285970 DO, DATA WGK ( 20), 0.0434525397 01356069316831728117 073 DO, DATA WGK ( 21), 0.044814800133162663192355551616723 DO , DATA WGK ( 22) , 0.0460592382 71006988116271735559374 DO, DATA WGK ( 23), 0.0471855465 69299153945261478181 099 DO, DATA WGK ( 24), 0.048185861757087129140779492298305 DO, DATA WGK ( 25) / 0.049055434555029778887528165367238 DO, DATA WGK ( 26), 0.049795683427074206357811569379942 DO, IATA WGK ( 27) , 0.050405921402782346840893085653585 DO , DATA WGK ( 28) , 0.0508817958 98749606492297473049805 DO, DATA WGK ( 29) , 0.051221547849258772170656282604944 DO, DATA WGK ( 30), 0.0514261285 3745902593 3862879215781 DO, DATA WGK ( 31 ), 0.051494729429451567558340433647099 DO, LIST OF MAJOR UARIABLES

CENTR - MID POINT OF THE INTERUAL HLGTH - HALF-LENGTH OF THE INTERUAL DABSC - ABSCISSA FUAL* - FUNCTION UALUE RESG - RESULT OF THE 30-POINT GAUSS RULE 


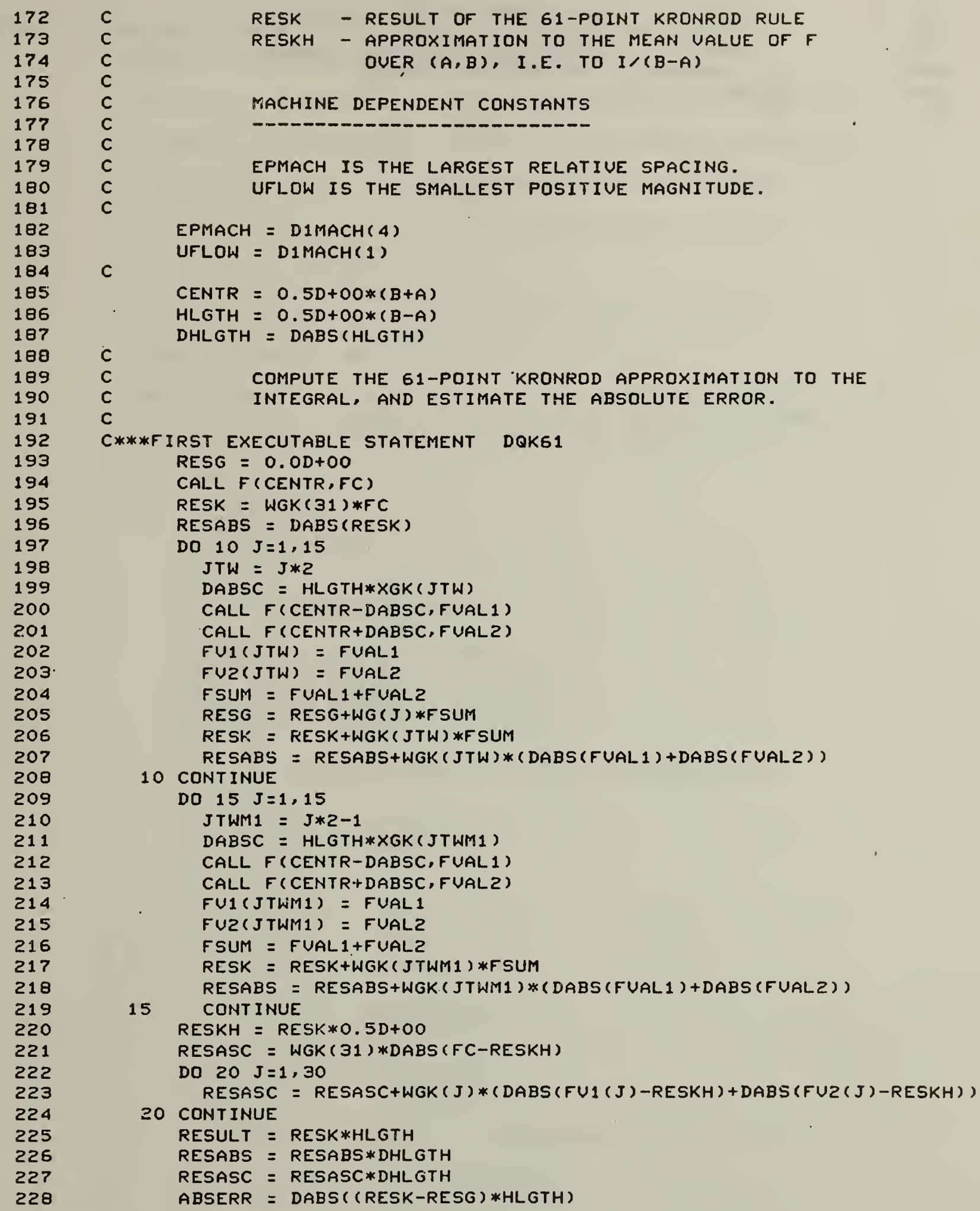

EPMACH IS THE LARGEST RELATIUE SPACING.

UFLOW IS THE SMALLEST POSITIUE MAGNITUDE.

c

c

$$
\text { EPMACH }=\text { D1MACH (4) }
$$

CENTR $=0.5 D+00 *(B+A)$

HLGTH $=0.5 D+00 *(B-A)$

DHLGTH $=$ DABS (HLGTH)

c

c

COMPUTE THE 61-POINT KRONROD APPROXIMATION TO THE INTEGRAL, AND ESTIMATE THE ABSOLUTE ERROR.

\section{C***FIRST EXECUTABLE STATEMENT DQKG1}

RESG $=0.0 D+O 0$

CALL F (CENTR,FC)

RESK = WGK (31)*FC

RESABS $=$ DABS (RESK)

DO $10 \mathrm{~J}=1,15$

$J T W=J * 2$

DABSC $=$ HLGTH*XGK (JTW)

CALL F (CENTR-DABSC, FUAL 1)

CALL $F(C E N T R+D A B S C, F \cup A L 2)$

FU1 (JTW) = FUAL1

$F \cup Z(J T W)=F U A L Z$

FSUM = FUAL1 +FUAL2

RESG $=$ RESG+WG ( $J$ ) *FSUM

RESK $=$ RESK+WGK $(J T W) * F S U M$

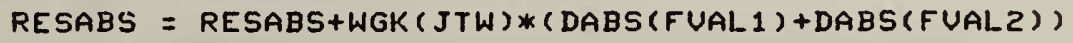

10 CONTINUE

DO $15 \mathrm{~J}=1,15$

$J$ TWM1 $=J * 2-1$

DABSC $=$ HLGTH*XGK (JTWM1)

CALL F (CENTR-DABSC,FUAL 1)

CALL F (CENTR+DABSC,FUAL2)

FUI (JTWM1) = FUAL 1

FUZ (JTWM1) = FUAL2

FSUM = FUAL1 +FUAL2

RESK $=$ RESK+WGK (JTWM1)*FSUM

RESABS = RESABS+WGK(JTWM1)*(DABS(FUAL1)+DABS (FUALZ) $)$

15 CONT INUE

RESKH $=$ RESK*0.5D+00

RESASC $=$ WGK ( 31$) * D A B S(F C-R E S K H)$

DO $20 \mathrm{~J}=1,30$

RESASC $=\operatorname{RESASC}+W G K(J) *(D A B S(F \cup 1(J)-R E S K H)+\operatorname{DABS}(F \cup Z(J)-R E S K H))$

20 CONTINUE

RESULT = RESK*HLGTH

RESABS = RESABS*DHLGTH

RESASC = RESASC $*$ DHLGTH

ABSERR $=$ DABS ( (RESK-RESG)*HLGTH) 
IF (RESASC. NE. O. OD+OO. AND. ABSERR. NE, O.OD+OO)

* abserR = RESASC*DMIN1(0.1 D+01.(0.2D+03*ABSERR/RESASC)* 1.5D+00) IF (RESABS. GT.UFLOW/ $(0.5 D+02 * E P M A C H))$ ABSERR = DMAX1

* ( (EPMACH*0.5D+02)*RESABS, ABSERR)

RETURN

END 
DOUBLE PRECISION FUNCTION DIMACH(I)

DOUBLE-PRECISION MACHINE CONSTANTS

D1MACH( 1) = B**(EMIN-1), THE SMALLEST POSITIUE MAGNITUDE.

DIMACH( 2) = B**EMAX*(1-B**(-T)), THE LARGEST MAGNITUDE.

DIMACH ( 3) = B**(-T), THE SMALLEST RELATIUE SPACING.

DIMACH $(4)=8 * *(1-T)$, THE LARGEST RELATIUE SPACING.

DIMACH ( 5) = LOG1O(B)

TO ALTER THIS FUNCTION FOR A PARTICULAR ENUIRONMENT, THE DESIRED SET OF DATA STATEMENTS SHOULD BE ACTIUATED BY REMOUING THE C FROM COLUMN 1.

WHERE POSSIBLE, OCTAL OR HEXADECIMAL CONSTANTS HAUE BEEN USED TO SPECIFY THE CONSTANTS EXACTLY WHICH HAS IN SOME CASES REQUIRED THE USE OF EQUIUALENT INTEGER ARRAYS.

INTEGER SMALL(4)

INTEGER LARGE(4)

INTEGER RIGHT( 4 )

INTEGER DIUER ( 4 )

INTEGER LOG1O(4)

DOUBLE PRECISION DMACH(5)

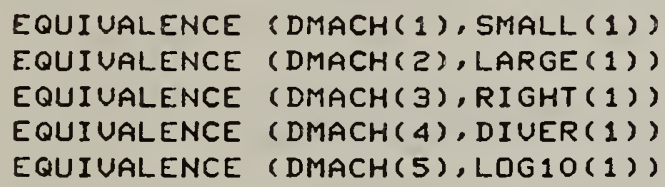

c

MACHINE CONSTANTS FOR THE BURROUGHS 1700 SYSTEM.

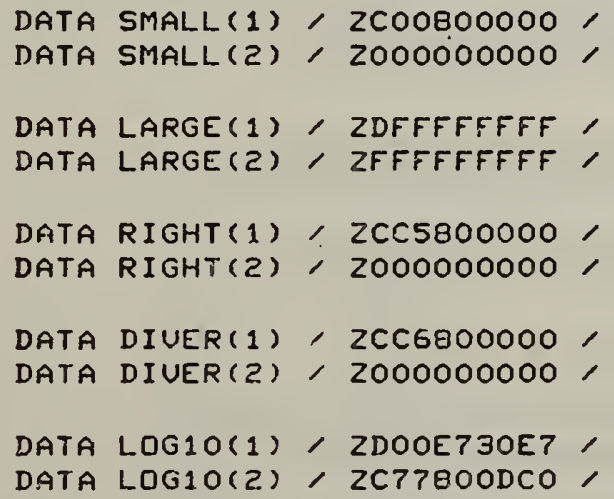




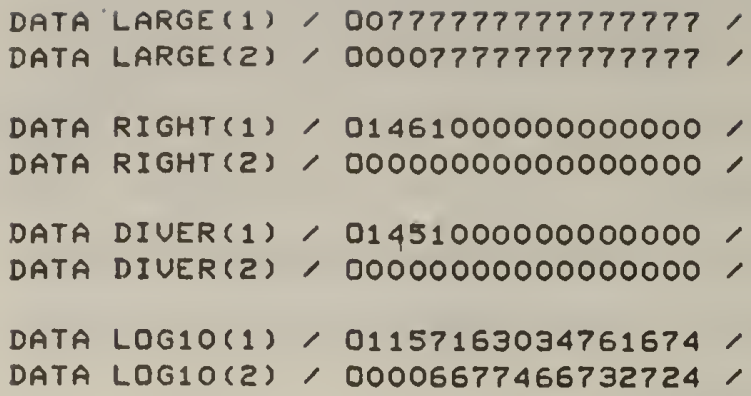




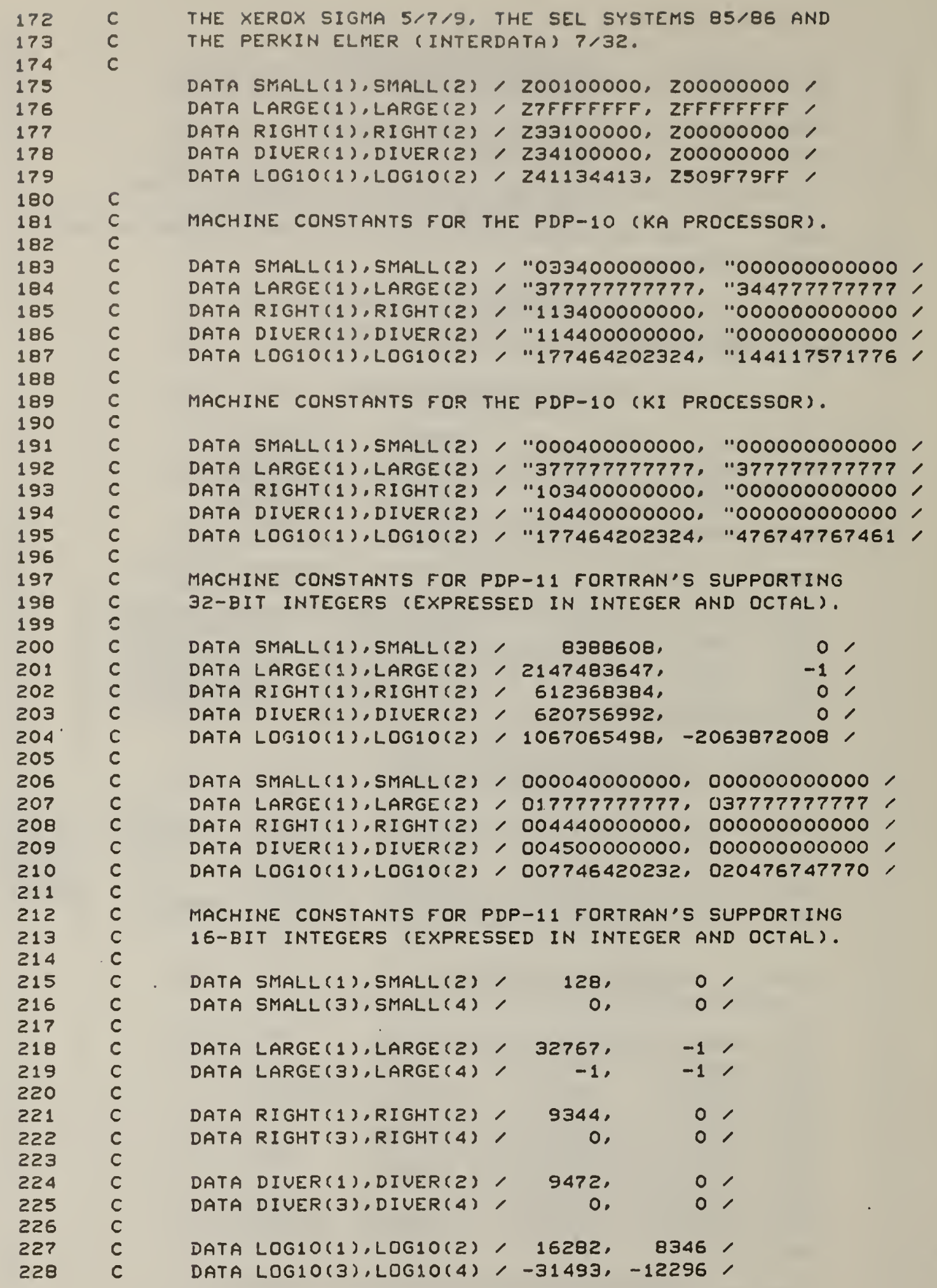




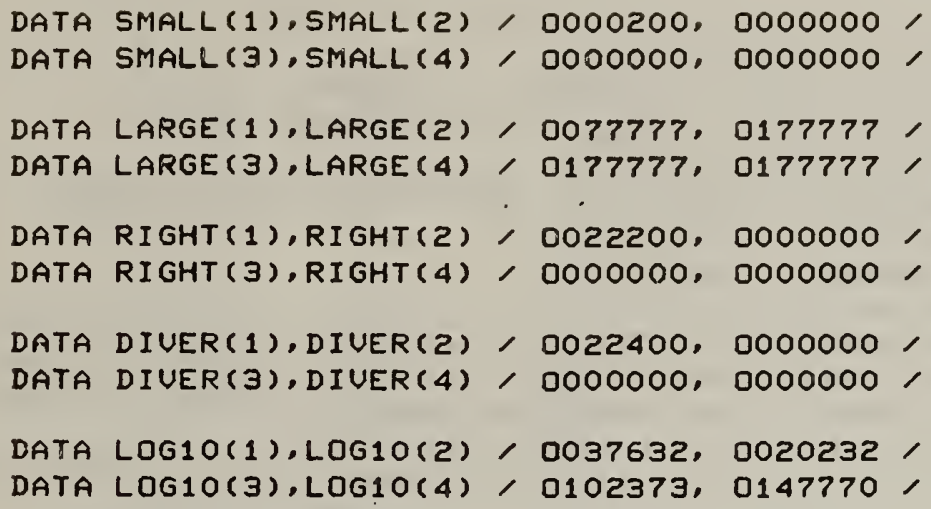

c

END 
STITL PWAFFI -- Initialize form-factor table for C-12 SUBROUTINE PWAFFI

IMPLICIT DOUBLE PRECISION $(A-H, O-Z)$

C The size of the arrays in the common PWAFF PARAMETER (MNPT $=200$ )

$C$ The number of points in the interpolation table PARAMETER (NTBM $1=179$ )

C The number of points + 1

PARAMETER (NTB $=$ NTBM $1+1$ )

DOUBLE PRECISION XC1 $(100), X C 2(79), F C 1(100), F C 2(79), X(M N P T)$ DOUBLE PRECISION F (NTBM1), DF (NTBM1), A(MNPT), B (MNPT), C (MNPT) DOUBLE PRECISION D(MNPT), AP (MNPT), BP (MNPT), CP (MNPT), DP (MNPT) DOUBLE PRECISION XC(NTBMI), FC(NTBMI)

COMMON /CONST/ EM, EMZ, AM, HBARC, ALPH,PIE, ALPHA, RTD, DTR COMMON /PWAFF, NPT, $X, A, B, C, D, A P, B P, C P, D P$ COMMON /CORREC/IWTILD, WTIL1, WTIL2, ISOFT, SOFT 1 EQUIUALENCE (FC(1),FC1 (1)), (FC(101), FC2 (1)) EQUIUALENCE $(X C(1), X C 1(1)),(X C(101), X C 2(1))$

C

Table of $q$ and corresponding form factor from DWBA code. In principle this table could be read in rather than defined in dat a statements.

DATA XC1/5.3068D-02,1.0613D-01,1.5918D-01,2.1222D-01,2.6523D-01,

$+3.1822 D-01,3.7118 D-01,4.2410 D-01,4.7697 D-01,5.2980 D-01$,

$+5.8258 D-01,6.3530 D-01,6.8795 D-01,7.4054 D-01,7.9305 D-01$,

$+8.4549 D-01,8.9784 D-01,9.5010 D-01,1.0023 D+00,1.0543 D+00$,

$+1.1063 D+00,1.1582 D+00,1.2099 D+00,1.2615 D+00,1.3130 D+00$.

$+1.3644 D+00.1 .4156 D+00,1.4667 D+00.1 .5177 D+00.1 .5685 D+00$.

$+1.6191 D+00.1 .6696 D+00.1 .7199 D+00.1 .7701 D+00.1 .8201 D+00$.

$+1.8699 D+00,1.9195 D+00,1.9690 D+00,2.0182 D+00,2.0673 D+00$,

$+2.1162 D+00,2.1648 D+00,2.2133 D+00,2.2615 D+00.2 .3096 D+00$,

$+2.3574 D+00.2 .4050 D+00.2 .4524 D+00.2 .4995 D+00.2 .5464 D+00$,

$+2.5931 D+00.2 .6395 D+00.2 .6856 D+00,2.7316 D+00,2.7772 D+00$,

$+2.8226 D+00.2 .8678 D+00,2.9127 D+00.2 .9573 D+00.3 .0017 D+00$,

$+3.0457 D+00,3.0895 D+00.3 .1331 D+00.3 .1763 D+00.3 .2193 D+00$,

$+3.2619 D+00,3.3043 D+00,3.3464 D+00,3.3881 D+00,3.4296 D+00$,

$+3.4708 D+00,3.5116 D+00.3 .5522 D+00.3 .5924 D+00,3.6324 D+00$,

$+3.6720 D+00,3.7113 D+00,3.7502 D+00,3.7889 D+00,3.8272 D+00$,

$+3.8652 D+00,3.9029 D+00,3.9402 D+00,3.9772 D+00,4.0138 D+00$,

$+4.0501 D+00,4.08 E 1 D+00,4.1218 D+00.4 .1570 D+00,4.1920 D+00$,

$+4.2266 D+00,4.2608 D+00,4.2947 D+00,4.3282 D+00,4.3614 D+00$,

$+4.3942 D+00.4 .4267 D+00.4 .4588 D+00.4 .4906 D+00.4 .5219 D+00$,

DATA $X C 2 / 4.5529 D+00,4.5836 D+00,4.6139 D+00,4.6438 D+00,4.6733 D+00$,

$+4.7025 D+00,4.7313 D+00,4.7598 D+00,4.7878 D+00,4.8155 D+00$,

$+4.8428 D+00.4 .8698 D+00.4 .8963 D+00,4.9225 D+00,4.9483 D+00$.

$+4.9737 D+00,4.9987 D+00.5 .0234 D+00.5 .0477 D+00.5 .0715 D+00$,

$+5.0950 D+00,5.1182 D+00,5.1409 D+00.5 .1632 D+00,5.1852 D+00$,

$+5.2068 D+00,5.2280 D+00.5 .2488 D+00,5.2692 D+00.5 .2892 D+00$,

$+5.3088 D+00,5.3280 D+00,5.3469 D+00,5.3653 D+00,5.3834 D+00$,

$+5.4011 D+00,5.4183 D+00.5 .4352 D+00.5 .4517 D+00.5 .4678 D+00$,

$+5.4835 D+00,5.4988 D+00.5 .5137 D+00.5 .5283 D+00.5 .5424 D+00$,

$+5.5561 D+00,5.5695 D+00,5.5824 D+00,5.5949 D+00,5.6071 D+00$,

$+5.6188 D+00,5.6302 D+00,5.6411 D+00,5.6517 D+00,5.6618 D+00$,

$+5.6716 D+00,5.681 O D+00.5 .6899 D+00.5 .6985 D+00,5.7067 D+00$,

$+5.7145 D+00,5.7218 D+00,5.7288 D+00,5.7354 D+00,5.7416 D+00$,

$+5.7474 D+00,5.7527 D+00,5.7577 D+00,5.7623 D+00,5.7665 D+00$,

$+5.7703 D+00,5.7737 D+00,5.7767 D+00,5.7793 D+00,5.7815 D+00$, 
$+5.7833 D+00,5.7847 D+00,5.7857 D+00,5.7862 D+00 /$ DATA FC1/1.0000D+00,9.8087D-01,9.5239D-01,9.1556D-01,8.6975D-01,

$+8.1646 \mathrm{D}-01,7.5736 \mathrm{D}-01,6.9420 \mathrm{D}-01,6.2873 \mathrm{D}-01,5.6261 \mathrm{D}-01$,

$+4.9734 \mathrm{D}-01,4.3427 \mathrm{D}-01,3.7450 \mathrm{D}-01,3.1890 \mathrm{D}-01,2.6807 \mathrm{D}-01$,

$+2.2240 D-01,1.8203 D-01.1 .4692 D-01,1.1688 D-01.9 .1572 D-02$,

$+7.0602 \mathrm{D}-02,5.3507 \mathrm{D}-02,3.9805 \mathrm{D}-02,2.9015 \mathrm{D}-02,2.0675 \mathrm{D}-02$,

$+1.4357 \mathrm{D}-02,9.6756 \mathrm{D}-03,6.2920 \mathrm{D}-03,3.9161 \mathrm{D}-03,2.3048 \mathrm{D}-03$,

$+1.2588 D-03,6.1895 D-04,2.6065 D-04,8.9112 D-05,3.4238 D-05$,

$+4.5899 D-05,8.9708 D-05,1.4332 D-04,1.9333 D-04,2.3265 D-04$,

$+2.5852 D-04,2.7093 D-04,2.7143 D-04,2.6234 D-04,2.4618 D-04$,

$+2.2533 D-04,2.0186 \mathrm{D}-04,1.7744 \mathrm{D}-04,1.5334 \mathrm{D}-04,1.3047 \mathrm{D}-04$,

$+1.0941 D-04,9.0497 D-05,7.3864 D-05,5.9512 D-05,4.7338 D-05$,

$+3.7177 D-05,2.8825 D-05.2 .2064 D-05,1.6672 D-05,1.2435 D-05$,

$+9.1554 D-06,6.6544 D-06,4.7753 D-06,3.3842 D-06,2.3691 D-06$,

$+1.6387 \mathrm{D}-06,1.1202 \mathrm{D}-06,7.5662 \mathrm{D}-07,5.0471 \mathrm{D}-07,3.3205 \mathrm{D}-07$,

$+2.1493 \mathrm{D}-07,1.3630 \mathrm{D}-07,8.4139 \mathrm{D}-08,5.0045 \mathrm{D}-08,2.8251 \mathrm{D}-08$,

$+1.4815 \mathrm{D}-08,7.0487 \mathrm{D}-09,3.1135 \mathrm{D}-09,1.7327 \mathrm{D}-09,2.0021 \mathrm{D}-09$,

$+3.2652 \mathrm{D}-09,5.0351 \mathrm{D}-09,6.9478 \mathrm{D}-09,8.7363 \mathrm{D}-09,1.0215 \mathrm{D}-08$,

$+1.1272 \mathrm{D}-08,1.1855 \mathrm{D}-0 \mathrm{~B}, 1.1969 \mathrm{D}-0 \mathrm{~B}, 1.1660 \mathrm{D}-08,1.1001 \mathrm{D}-08$,

$+1.0085 D-08,9.0060 D-09,7.8529 D-09,6.7016 D-09,5.6109 D-09$,

$+4.6210 \mathrm{D}-09,3.7547 \mathrm{D}-09,3.0203 \mathrm{D}-09,2.4145 \mathrm{D}-09,1.9268 \mathrm{D}-09 /$ DATA FC2/1.5422D-09,1.2443D-09,1.0168D-09,8.4509D-10,7.1668D-10, $+6.2124 D-10,5.5047 D-10.4 .9785 D-10.4 .5831 D-10,4.2782 D-10$,

$+4.0331 \mathrm{D}-10,3.8236 \mathrm{D}-10,3.6319 \mathrm{D}-10,3.4448 \mathrm{D}-10,3.2538 \mathrm{D}-10$,

$+3.0541 \mathrm{D}-10,2.8440 \mathrm{D}-10,2.6246 \mathrm{D}-10,2.3986 \mathrm{D}-10,2.1698 \mathrm{D}-10$,

$+1.9427 \mathrm{D}-10,1.7217 \mathrm{D}-10,1.5108 \mathrm{D}-10,1.3133 \mathrm{D}-10,1.1315 \mathrm{D}-10$,

$+9.6708 D-11,8.2061 D-11.6 .9205 D-11,5.8070 D-11,4.8544 D-11$,

$+4.0484 D-11.3 .3732 D-11,2.8123 D-11,2.3498 D-11,1.9707 D-11$,

$+1.6614 \mathrm{D}-11.1 .4098 \mathrm{D}-11.1 .2055 \mathrm{D}-11.1 .0398 \mathrm{D}-11.9 .0514 \mathrm{D}-12$,

$+7.9547 D-12,7.0577 D-12,6.3195 D-12,5.7071 D-12,5.1943 D-12$,

$+4.7601 D-12,4.3877 D-12,4.0639 D-12,3.7782 D-12.3 .5222 D-12$,

$+3.2896 \mathrm{D}-12,3.0756 \mathrm{D}-12,2.8765 \mathrm{D}-12,2.6900 \mathrm{D}-12,2.5143 \mathrm{D}-12$,

$+2.3433 D-12,2.1911 D-12,2.0422 D-12,1.9009 D-12,1.7673 D-12$,

$+1.6412 D-12,1.5229 D-12,1.4126 D-12,1.3105 D-12,1.2164 D-12$,

$+1.1302 D-12,1.0513 D-12,9.7921 D-13,9.1362 D-13,8.5462 D-13$,

$+8.0261 \mathrm{D}-13,7.5814 \mathrm{D}-13, ? .2158 \mathrm{D}-13,6.9264 \mathrm{D}-13,6.6982 \mathrm{D}-13$.

$+6.4928 D-13,6.2152 D-13,5.5475 D-13,3.2798 D-13 /$

C

C

10

c

Convert table from form factor versus $q$ to form iactor $t$ imes $2 * * 2$ versus $q * * 2$

DO $10 \quad I=1, N T B M 1$

$X(I)=X C(I) * * 2$

$F(I)=F C(I) * 36$. DO

IF (IWTILD.NE.O) $F(I)=F(I) *(W T I L 1+W T I L 2 * D L O G(X(I) / E M 2)$ ) CONT INUE

Find the cubic coefficients for spline interpolation of the table. A good reference for spline interpolation is Elementary numerical analysis by S.D. Conte and Carl deBoor. $A(1)=36$. DO

$B(1)=0 . D O$

$C(1)=0 . D O$

$D(1)=0 . D O$

$X(N T B)=1 . D 74$

$A(N T B)=0 . D O$

$B(N T B)=0 . D O$

$C(N T B)=0 . D O$ 


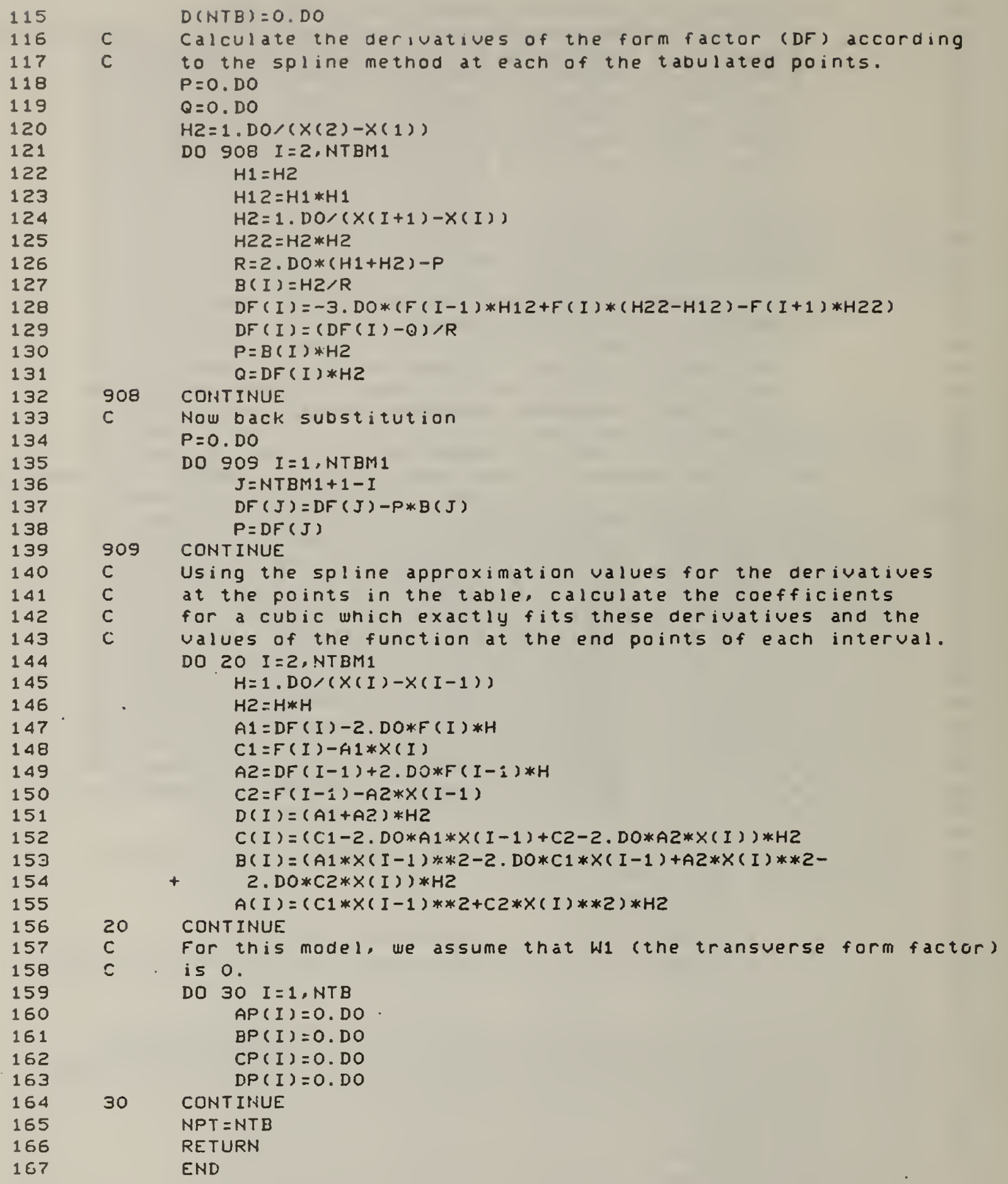




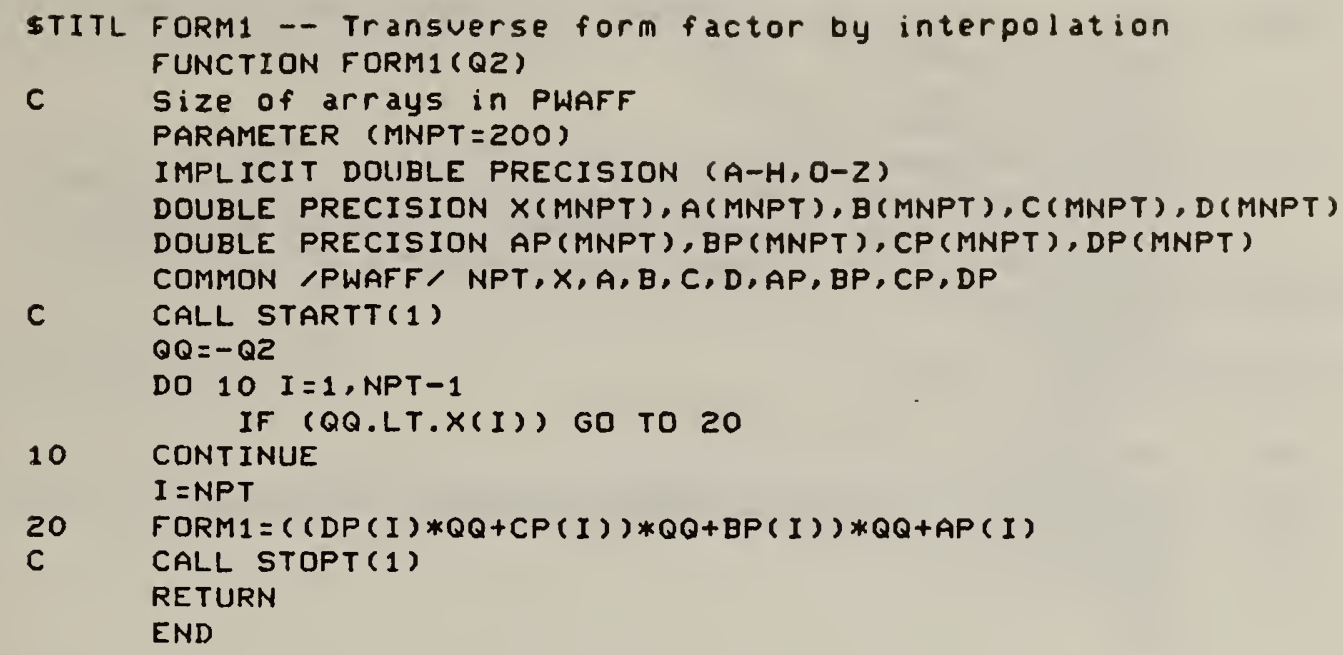




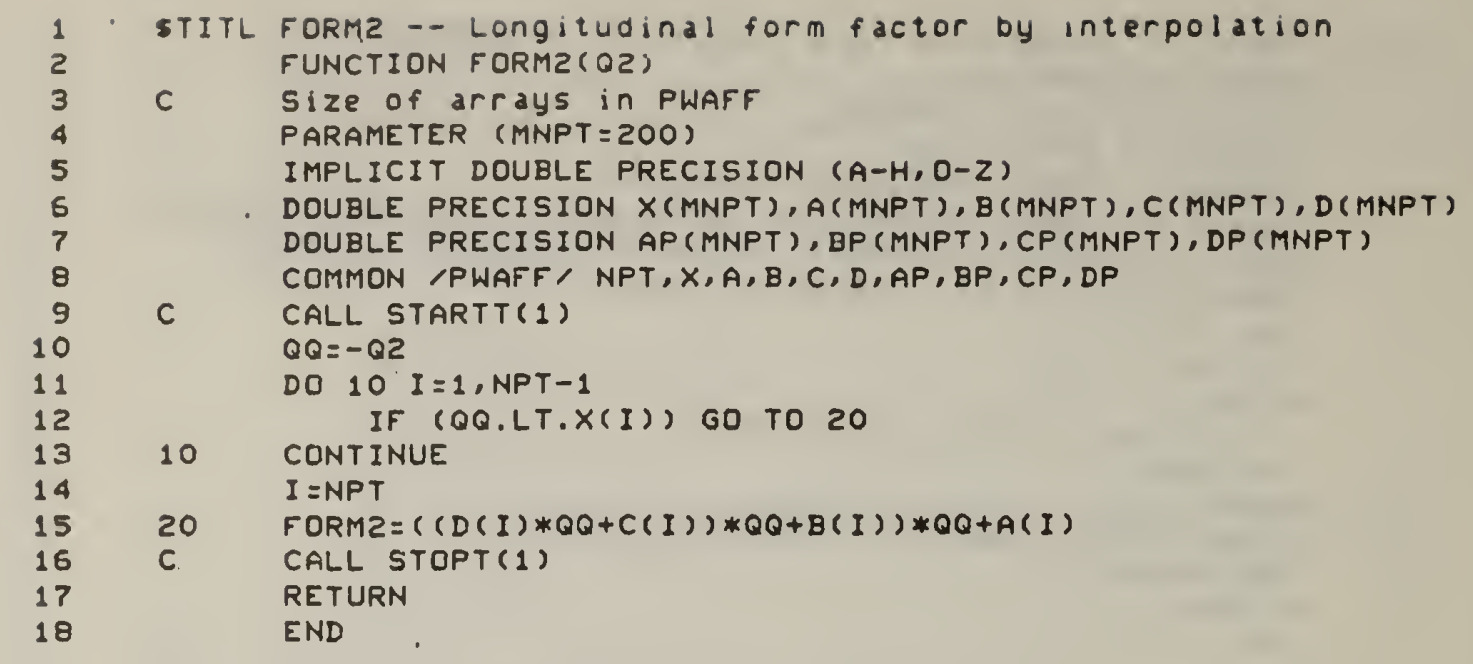


4. TITLE AND SUBTITLE

PIECE-WISE ANALYTIC EVALUATION OF THE RADIATIVE TAIL

FROM ELASTIC AND INELASTIC ELECTRON SCATTERING

5. $A \cup T H O R(S)$

Leonard C. Maximon and Steven E. Williamson

6. PERFORMING ORGANIZATION (If jolnt or other than NBS, see instructlons)

7. Contract/Grant No.

NATIONAL BUREAU OF STANDARDS

DEPARTMENT OF COMMERCE

WASHINGTON, D.C. 20234

9. SPONSORING ORGANIZATION NAME AND COMPLETE ADDRESS (Street, City, State, ZIP)

Document describes a computer program; SF-185, FIPS Software Summary, is attached.

11. ABSTRACT (A 200-word or less foctual summary of most significant informotion. If document includes a signlficant bibliography or literoture survey, mention it here)

We consider here the calculation of the radiative tail from the elastic peak in medium and high energy electron scattering as well as from a discrete inelastic level of the recoiling nucleus. We examine the method generally used for this calculation, viz., a numerical integration of the differential cross section over the angles of the unobserved photon, and discuss the difficulties inherent in this numerical integration due to the sharp peaking of the integrand. We present an alternative method for calculating the radiative tail, in which the region of integration is divided into an arbitrary number of subintervals, the structure functions are fitted by cubic spline functions in each subinterval, and the integrations are then performed analytically in closed form. This method has the advantages of greatly increased accuracy and a reduction of the computation time by a factor which can vary between 10 and $10^{3}$, depending on the kinematics.

12. KEY WORDS (Six to twelve entries; alphabetical order; capitalize only proper names; and separate key words by semicolons) Key words: electron scattering cross sections; elastic electron scattering; elastic radiative tail; inelastic electron scattering; inelastic radiative tail; intermediate and high energy electron scattering.

13. AVAILABILITY

[] Unlimited

For Official Distribution. Do Not Release to NTIS

$\square$ Order From Superintendent of Documents, U.S. Government Printing Office, Washington, D.C. 20402.

14. NO. OF PRINTED PAGES

区] Order From National Technical Information Service (NTIS), Springfield, VA. 2216I
15. Price

$\$ 13.00$ 



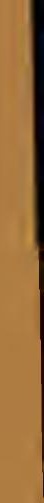


